\title{
A Riemann solver for single-phase and two-phase shallow flow models based on relaxation. Relations with Roe and VFRoe solvers
}

\author{
Marica Pelanti a,*, François Bouchut ${ }^{\mathrm{b}, 1}$, Anne Mangeney ${ }^{\mathrm{c}}$ \\ a Département de Mathématiques et Applications, École Normale Supérieure, 45, rue d'Ulm - 75230 Paris cedex 05, France \\ ${ }^{\mathrm{b}}$ CNRS and Département de Mathématiques et Applications, École Normale Supérieure, 45, rue d'Ulm - 75230 Paris cedex 05, France \\ ${ }^{\mathrm{c}}$ Équipe de Sismologie, Institut de Physique du Globe de Paris, 4, place Jussieu - 75252 Paris cedex 05, France
}

\section{A R T I C L E I N F O}

\section{Article history:}

Received 7 January 2010

Received in revised form 23 August 2010

Accepted 4 October 2010

Available online $\mathrm{xxxx}$

\section{Keywords:}

Shallow flow models

Two-phase granular flows

Riemann solvers

Relaxation

Positivity

Wet/dry fronts

\begin{abstract}
A B S T R A C T
We present a Riemann solver derived by a relaxation technique for classical single-phase shallow flow equations and for a two-phase shallow flow model describing a mixture of solid granular material and fluid. Our primary interest is the numerical approximation of this two-phase solid/fluid model, whose complexity poses numerical difficulties that cannot be efficiently addressed by existing solvers. In particular, we are concerned with ensuring a robust treatment of dry bed states. The relaxation system used by the proposed solver is formulated by introducing auxiliary variables that replace the momenta in the spatial gradients of the original model systems. The resulting relaxation solver is related to Roe solver in that its Riemann solution for the flow height and relaxation variables is formally computed as Roe's Riemann solution. The relaxation solver has the advantage of a certain degree of freedom in the specification of the wave structure through the choice of the relaxation parameters. This flexibility can be exploited to handle robustly vacuum states, which is a well known difficulty of standard Roe's method, while maintaining Roe's low diffusivity. For the single-phase model positivity of flow height is rigorously preserved. For the two-phase model positivity of volume fractions in general is not ensured, and a suitable restriction on the CFL number might be needed. Nonetheless, numerical experiments suggest that the proposed two-phase flow solver efficiently models wet/dry fronts and vacuum formation for a large range of flow conditions.

As a corollary of our study, we show that for single-phase shallow flow equations the relaxation solver is formally equivalent to the VFRoe solver with conservative variables of Gallouët and Masella [T. Gallouët, J.-M. Masella, Un schéma de Godunov approché C.R. Acad. Sci. Paris, Série I, 323 (1996) 77-84]. The relaxation interpretation allows establishing positivity conditions for this VFRoe method.
\end{abstract}

(c) 2010 Elsevier Inc. All rights reserved.

\section{Introduction}

This paper illustrates a wave propagation finite volume scheme for single-phase and two-phase shallow flow models obtained by a relaxation strategy. Our primary interest is the numerical approximation of a two-phase depth-averaged model

\footnotetext{
* Corresponding author. Present address: INRIA Paris-Rocquencourt, Team BANG, Domaine de Voluceau, 78153 Le Chesnay, France. Laboratoire National d'Hydraulique et Environnement, EDF R\&D, 6, quai Watier, 78401 Chatou, France.

E-mail addresses: Marica.Pelanti@ens.fr, marica.pelanti@inria.fr (M. Pelanti), francois.bouchut@univ-mlv.fr, Francois.Bouchut@ens.fr (F. Bouchut), mangeney@ipgp.jussieu.fr (A. Mangeney).

1 Present address: Laboratoire d'Analyse et de Mathématiques Appliquées, Université Paris-Est Marne-la-Vallée, 5, boulevard Descartes, Cité Descartes Champs-sur-Marne, 77454 Marne-la-Vallée cedex 2, France.
} 
describing the flow of a shallow layer of a mixture of solid granular material and fluid. The model system is a variant of the Pitman-Le two-fluid model [1], and it consists of mass and momentum equations for the solid and fluid phases, coupled together by both conservative and non-conservative momentum exchange terms. The main application of interest of this twophase model is the simulation of geophysical gravitational flows such as avalanches and debris flows, which typically contain both solid granular components and an interstitial fluid phase. Indeed, most of the models used to simulate real avalanches do not take into account the presence of a fluid component (e.g. [2-4]). The fluid however is expected to play a key role in the mobility of natural flows and in the structure of their deposits [5-7]. Furthermore, interpretation of the deposits of gravitational flows on Mars in terms of fluid content is a key issue in planetary sciences because of its connection to life [8,7].

The considered two-phase granular flow model was first studied in $[9,10]$, where it was shown to be hyperbolic under the condition of phase velocity differences sufficiently small. The hyperbolicity assumption is then reasonable for the foreseen applications to geophysical flows, where usually inter-phase forces rapidly drive solid and fluid constituents towards kinematic equilibrium [5]. In [9,10] the two-phase granular flow system was numerically approximated by a Godunov-type finite volume scheme based on a Roe-type Riemann solver. One disadvantage of this Roe-type method is that it may produce unphysical negative discrete values of the flow depth and of phase volume fractions. Positivity preservation of flow height is a fundamental property for numerical models for free surface flows to handle correctly wet/dry transition zones where the flow height vanishes (wet/dry fronts). Here in particular we are interested in the computation of these flows by finite volume schemes based on Riemann solvers [11-14]. We refer for instance to [15] for a survey of other approaches.

For classical single-phase shallow water equations numerous positivity preserving Riemann solvers are available. First, there are classical robust methods such as the exact Riemann solver (Godunov method [16]) and the HLL, HLLC solvers $[17,14]$. Other approximate solvers able to treat efficiently vacuum states have been developed by means of relaxation strategies, such as Suliciu's solver [18-20], and the recent method of Berthon-Marche [21]. Among other methods, let us mention the augmented four-wave Riemann solver of George [22], which is related to the class of relaxation solvers of LeVeque and Pelanti [23], and the modified Roe method (MRoe) of Castro, Parés and co-workers [24,25]. This MRoe method however is not rigorously positivity preserving, and it may need a restriction on the CFL number to avoid negative water depths.

Unfortunately, it is difficult to extend the existing positivity preserving techniques for single-phase shallow flow equations to the considered two-phase granular flow model, due to its non-conservative character and the complexity of its Riemann solution structure. Major difficulties for the two-phase system are the lack of explicit expressions of the eigenvalues, the limited information about the structure of the characteristic fields (Riemann invariants are not available), and hence the lack of knowledge of the exact Riemann solution. The presence of non-conservative terms prevents resorting to methods of the HLL family [17].

In an effort to build a robust method for the two-phase model suited for flow regimes involving dry bed regions, we have studied a new Riemann solver derived through a relaxation technique. Our work has been particularly inspired by the relaxation approach of Berthon and Marche [21] for shallow water equations, although here we develop a new idea, and our relaxation system does not enter in the class of relaxation models proposed in [21]. In [21] the authors establish positivity of the VFRoe scheme with non-conservative variables (VFRoe-ncv) of [26-28] by identifying the VFRoe-ncv solver with a particular relaxation solver. The positivity preserving approach of Berthon-Marche relies on a choice of relaxation variables that is motivated by the form of the Riemann invariants of the single-phase shallow flow system. The technique then is not directly applicable to our two-phase system, for which the Riemann invariants are not known, and there is no analogous form of the relaxation model useful to enforce positivity in the two-phase case by the same arguments as in [21]. The relaxation method that we introduce here has originated after initial attempts of extending the Berthon-Marche approach to the two-phase system. Both our relaxation model and the one in [21] are based on the pioneering idea of Jin and Xin [29] of approximating the original model equations via a new system that is easier to solve (see also e.g. [30,31,23]). The particular feature that we have borrowed from Berthon and Marche [21] is the formulation of a relaxation system with linear degeneracy in all the characteristic fields, a property obtained by a special decoupling of the linear equations governing the relaxation variables from the remaining non-linear equations of the relaxation model. The innovative idea of our model is to introduce auxiliary variables that replace the momenta in the spatial gradients of the original single-phase and two-phase systems. These new variables are governed by linear equations with coefficients that determine the eigenstructure of the relaxation model, similar to [21]. This procedure leads to a Riemann solver that is related to Roe solver in that its Riemann solution for the flow height and relaxation variables is formally computed as Roe's Riemann solution. The relaxation solver has the advantage of a certain degree of freedom in the specification of the wave structure through the choice of the relaxation parameters. This flexibility can be exploited to handle robustly dry bed states and vacuum appearance, while maintaining Roe's method low diffusivity and sharp shock resolution. For the single-phase system positivity of flow height is rigorously preserved. For the two-phase model positivity in general is not ensured, and a suitable restriction on the CFL number might be needed. Nonetheless, numerical experiments suggest that the proposed two-phase flow solver efficiently models wet/dry fronts and vacuum formation for a large range of flow conditions, often with no need of a CFL reduction.

As a corollary of our study, in analogy with the results of Berthon-Marche [21] for the VFRoe-ncv method, we find formal equivalence of our relaxation solver for single-phase shallow flows with the first original version of the VFRoe solver, the method with conservative variables of [32,33]. Similar to [21], the relaxation interpretation allows establishing positivity conditions for this version of the VFRoe scheme.

The paper is structured as follows. First, in Sections 2 and 3 we present the shallow flow models under study. We then give a brief presentation of wave propagation algorithms based on Riemann solvers in Section 4, recalling Roe [34] and 
Roe-type solvers and the difficulties in handling vacuum states encountered by these methods. In Section 5 we present our relaxation solver for the single-phase case, and in Section 6 we discuss its equivalence with the VFRoe solver of [32,33]. The relaxation solver for the two-phase granular flow model is illustrated in Section 7. Numerical results for both the singlephase and the two-phase model are presented in Section 8, and some concluding remarks are finally written in Section 9. Appendix A is not directly related to the relaxation approach, but it is dedicated to a positivity preserving Roe method for the single-phase shallow flow system.

\section{Single-phase shallow flow equations}

The classical one-dimensional single-phase shallow water system has the conservative form

$$
\begin{aligned}
& \partial_{t} h+\partial_{x} m=0, \\
& \partial_{t} m+\partial_{x}\left(\frac{m^{2}}{h}+\frac{g}{2} h^{2}\right)=0,
\end{aligned}
$$

where $h$ is the flow height, $m=h u$ the momentum, and $u$ the flow velocity in the $x$ direction. In compact form:

$$
\partial_{t} q+\partial_{x} f(q)=0,
$$

where

$$
q=\left(\begin{array}{c}
h \\
m
\end{array}\right) \text { and } f(q)=\left(\begin{array}{c}
m \\
\frac{m^{2}}{h}+\frac{g}{2} h^{2}
\end{array}\right)
$$

denote the vector of the conserved variables and the flux vector function, respectively. The quasi-linear form of the equations is

$$
\begin{aligned}
& \partial_{t} q+A(q) \partial_{x} q=0, \\
& A(q)=f^{\prime}(q)=\left(\begin{array}{cc}
0 & 1 \\
-u^{2}+g h & 2 u
\end{array}\right),
\end{aligned}
$$

and the system is defined over the convex set of admissible states

$$
\Omega=\left\{q \in \mathbb{R}^{2} ; h \geqslant 0, u \in \mathbb{R}\right\} .
$$

The system has eigenvalues $\lambda_{k}$ and corresponding right eigenvectors $r_{k}, k=1,2$, given by

$$
\lambda_{1,2}=u \mp c, \quad r_{1,2}=\left(\begin{array}{c}
1 \\
u \mp c
\end{array}\right), \quad c=\sqrt{g h},
$$

and it is strictly hyperbolic for $h>0$. The left eigenvectors can be taken as

$$
l_{1,2}=\frac{1}{2 c}( \pm u+c, \mp 1)
$$

with the normalization $l_{j} r_{k}=\delta_{j k}$ (Kronecker's delta).

\section{A two-phase shallow granular flow model}

We consider a shallow layer of a mixture of solid granular material and fluid over a horizontal surface. Solid and fluid components are assumed incompressible, with constant specific densities $\rho_{s}$ and $\rho_{f}<\rho_{s}$, respectively. We denote with $h$ the flow height and with $\varphi$ the solid volume fraction, and we define the variables

$$
h_{s}=\varphi h \quad \text { and } \quad h_{f}=(1-\varphi) h .
$$

We consider the one-dimensional case, and we indicate solid and fluid velocities in the $x$ direction with $u_{s}, u_{f}$, respectively. Phase momenta are given by $m_{s}=h_{s} u_{s}$ and $m_{f}=h_{f} u_{f}$. The dynamics of this granular mixture can be modeled by the following depth-averaged system, consisting of mass and momentum equations for the two constituents:

$$
\begin{aligned}
& \partial_{t} h_{s}+\partial_{x} m_{s}=0, \\
& \partial_{t} m_{s}+\partial_{x}\left(\frac{m_{s}^{2}}{h_{s}}+\frac{g}{2} h_{s}^{2}+g \frac{1-\gamma}{2} h_{s} h_{f}\right)+\gamma g h_{s} \partial_{x} h_{f}=\gamma F^{\mathrm{D}}, \\
& \partial_{t} h_{f}+\partial_{x} m_{f}=0, \\
& \partial_{t} m_{f}+\partial_{x}\left(\frac{m_{f}^{2}}{h_{f}}+\frac{g}{2} h_{f}^{2}\right)+g h_{f} \partial_{x} h_{s}=-F^{\mathrm{D}} .
\end{aligned}
$$

Above, $g$ is the gravity constant and 


$$
\gamma=\frac{\rho_{f}}{\rho_{s}}<1
$$

Source terms on the right-hand side of the momentum equations model inter-phase drag forces $F^{\mathrm{D}}$, which we express as $F^{\mathrm{D}}=D\left(h_{s}+h_{f}\right)\left(u_{f}-u_{s}\right)$, where $D=D\left(\varphi,\left|u_{f}-u_{s}\right| ; \sigma\right)$ is a drag function depending in general on $\varphi,\left|u_{f}-u_{s}\right|$, and a set of physical parameters $\sigma$ (e.g. specific densities, particle diameter). Drag effects in the model are important for maintaining flow conditions in the hyperbolic regime, as it will be clearer in the following (Section 3.1). However, in this paper we will only be concerned with the numerical approximation of the homogeneous system.

In compact form the homogeneous system reads

$$
\partial_{t} q+\partial_{x} f(q)+w\left(q, \partial_{x} q\right)=0
$$

where

$$
q=\left(\begin{array}{c}
h_{s} \\
m_{s} \\
h_{f} \\
m_{f}
\end{array}\right), \quad f(q)=\left(\begin{array}{c}
m_{s} \\
\frac{m_{s}^{2}}{h_{s}}+\frac{g}{2} h_{s}^{2}+g \frac{1-\gamma}{2} h_{s} h_{f} \\
m_{f} \\
\frac{m_{f}^{2}}{h_{f}}+\frac{g}{2} h_{f}^{2}
\end{array}\right), \quad \text { and } \quad w\left(q, \partial_{x} q\right)=\left(\begin{array}{c}
0 \\
\gamma g h_{s} \partial_{x} h_{f} \\
0 \\
g h_{f} \partial_{x} h_{s}
\end{array}\right) .
$$

Above, we have put into evidence the conservative portion of the system $\partial_{x} f(q)$, and the non-conservative term $w\left(q, \partial_{x} q\right)$. Note that the mass equations for $h_{s}$ and $h_{f}$ are conservative, whereas the momentum equations for $m_{s}$ and $m_{f}$ exhibit non-conservative products that couple the dynamics of the solid and fluid phases. The momentum of the mixture $m_{\mathrm{m}}=m_{s}+\gamma m_{f}$ is nonetheless conserved:

$$
\partial_{t} m_{\mathrm{m}}+\partial_{x} f_{\mathrm{m}}(q)=0
$$

where

$$
f_{\mathrm{m}}(q)=f^{(2)}(q)+\gamma f^{(4)}(q)+\gamma g h_{s} h_{f}=\frac{m_{s}^{2}}{h_{s}}+\gamma \frac{m_{f}^{2}}{h_{f}}+\frac{g}{2}\left(h_{s}^{2}+\gamma h_{f}^{2}\right)+g \frac{1+\gamma}{2} h_{s} h_{f} .
$$

Let us also write the homogeneous system in quasi-linear form for future reference. We have:

$$
\partial_{t} q+A(q) \partial_{x} q=0,
$$

where

$$
A(q)=\left(\begin{array}{cccc}
0 & 1 & 0 & 0 \\
-u_{s}^{2}+g h_{s}+g \frac{1-\gamma}{2} h_{f} & 2 u_{s} & g \frac{1+\gamma}{2} h_{s} & 0 \\
0 & 0 & 0 & 1 \\
g h_{f} & 0 & -u_{f}^{2}+g h_{f} & 2 u_{f}
\end{array}\right) .
$$

The set of admissible states for this model is:

$$
\Omega=\left\{q \in \mathbb{R}^{4} ; h_{s}, h_{f} \geqslant 0, u_{s}, u_{f} \in \mathbb{R}\right\},
$$

or, equivalently, in terms of $h=h_{s}+h_{f}$ and $\varphi=\frac{h_{s}}{h_{s}+h_{f}}$,

$$
\Omega=\left\{q \in \mathbb{R}^{4} ; h \geqslant 0, \varphi \in[0,1], u_{s}, u_{f} \in \mathbb{R}\right\} .
$$

The two-phase model (8) is a variant of the two-phase debris flow model of Pitman and Le [1]. It was previously studied in $[9,10]$ in an extended form that included topography terms accounting for a variable bottom surface. The model system above differs from the original work of Pitman and Le [1] in the description of the fluid and mixture momentum balance, and, in contrast with [1], has the property of recovering a conservative equation for the momentum of the mixture $m_{\mathrm{m}}$ as shown in (11). We recently learned that Le presented in his thesis work [35] a version of his earlier two-phase model [1] that results to be equivalent to our formulation. Le in his thesis analyzes the model system in normalized form and he claims hyperbolicity assuming "reasonable parameters" in the equations. In the next section we illustrate the results of the more precise eigenvalue analysis of the system that we presented in $[9,10]$.

\subsection{Eigenvalues and hyperbolicity}

In general, simple explicit expressions of the eigenvalues $\lambda_{k}, k=1, \ldots, 4$, of the matrix $A$ of the system cannot be derived. In the particular case of equality of solid and fluid velocities, $u_{f}=u_{s} \equiv u$, the eigenvalues are real and distinct $(h>0, \varphi \neq 1)$ and given by

$$
\lambda_{1,4}=u \mp a \quad \text { and } \quad \lambda_{2,3}=u \mp a \beta,
$$

where we have introduced the quantities

$$
a=\sqrt{g h} \text { and } \beta=\sqrt{(1-\varphi) \frac{1-\gamma}{2}}<1 .
$$


Other particular cases are: (i) $\varphi=0$, for which the eigenvalues are $u_{f} \mp a, u_{s} \mp a \beta$, with $\beta=\sqrt{\frac{1-\gamma}{2}}$; (ii) $\varphi=1$, for which we find the two distinct eigenvalues $u_{s} \mp a$ and the double eigenvalue $u_{f}$. For the general case, in [10] we proved the following:

Proposition 3.1. Matrix A (12b) has always at least two real eigenvalues $\lambda_{1,4}$, and moreover, the eigenvalues $\lambda_{k}$ of $A, k=1, \ldots, 4$, satisfy:

$$
u_{\min }-a \leqslant \lambda_{1} \leqslant \Re\left(\lambda_{2}\right) \leqslant \Re\left(\lambda_{3}\right) \leqslant \lambda_{4} \leqslant u_{\max }+a,
$$

where $u_{\min } \equiv \min \left(u_{f}, u_{s}\right), u_{\max } \equiv \max \left(u_{f}, u_{s}\right)$, and $\mathfrak{R}(\cdot)$ denotes the real part. Furthermore:

(i) If $\left|u_{s}-u_{f}\right| \leqslant 2 a \beta$ or $\left|u_{s}-u_{f}\right| \geqslant 2 a$ then all the eigenvalues are real. If one of these inequalities is strictly satisfied, and if $h>0$ and $\varphi \neq 1$, then the eigenvalues are also distinct, and system (12) is strictly hyperbolic.

(ii) If $2 a \beta<\left|u_{s}-u_{f}\right|<2 a$ then the internal eigenvalues $\lambda_{2,3}$ may be complex.

Proposition 3.1 shows that our model system is hyperbolic for differences of solid and fluid velocities either sufficiently small or sufficiently large, compared to the characteristic speeds of flow in kinematic equilibrium, and that there exists a range of values of the phase velocity difference for which the system eigenvalues may be complex and hyperbolicity is lost. The relevant hyperbolic regime for applications is the one corresponding to small $\left|u_{s}-u_{f}\right|$. It is understood that inter-phase drag forces act in favor of hyperbolic flow conditions, since they tend to drive phase velocities closer.

Let us also recall the bounding intervals that we found for the eigenvalues in [10], in addition to the bounds (16). For the external eigenvalues $\lambda_{1,4}$ we have

$$
\begin{aligned}
& \lambda_{1} \in\left(u_{\min }-a, \min \left(u_{\max }-a, u_{\min }-a \beta\right)\right) \text { and } \\
& \lambda_{4} \in\left(\max \left(u_{\min }+a, u_{\max }+a \beta\right), u_{\max }+a\right),
\end{aligned}
$$

and for the internal eigenvalues $\lambda_{2,3}$, if they are real, we have

$$
\begin{aligned}
& \lambda_{2} \in\left(\min \left(u_{\max }-a, u_{\min }-a \beta\right), \min \left(u_{\max }-a \beta, u_{\min }+a\right)\right) \quad \text { and } \\
& \lambda_{3} \in\left(\max \left(u_{\max }-a, u_{\min }+a \beta\right), \max \left(u_{\min }+a, u_{\max }+a \beta\right)\right) .
\end{aligned}
$$

\subsubsection{Implicit analytical expressions of the eigenvalues}

We write here implicit analytical expressions of the system's eigenvalues (not presented in [10]), since the formulas give some insight into the dependence on the flow variables, and they will be useful in the following (Section 7.3). In general, the eigenvalues have the form:

$$
\lambda_{1,4}=V_{\mathrm{e}} \mp c_{\mathrm{e}} \quad \text { and } \quad \lambda_{2,3}=V_{\mathrm{i}} \mp c_{\mathrm{i}}, \quad c_{\mathrm{e}}, c_{\mathrm{i}} \geqslant 0
$$

with

$$
V_{\mathrm{e}}=\bar{u}+d \quad \text { and } \quad V_{\mathrm{i}}=\bar{u}-d,
$$

where $\bar{u}=\frac{u_{s}+u_{f}}{2}$ and the $d$ represents the deviation of the velocities $V_{\mathrm{e}}$ and $V_{\mathrm{i}}$ of the external and internal eigenvalues, respectively, from the mean velocity $\bar{u}$. Let us define $\Delta U=u_{s}-u_{f}$. The quantities $c_{\mathrm{e}}$ and $c_{\mathrm{i}}$ can be expressed as

$$
c_{\mathrm{e}}=\sqrt{T+\sqrt{Z^{+}}} \text {and } c_{\mathrm{i}}=\sqrt{T-\sqrt{Z^{-}}},
$$

where

$$
\begin{aligned}
& T=\left(\frac{\Delta U}{2}\right)^{2}-3 d^{2}+\frac{1}{2} a^{2}(1+\beta)^{2}, \\
& Z^{ \pm}=\frac{1}{4} a^{4}\left(1-\beta^{2}\right)^{2} \pm a^{2}|d \| \Delta U|\left|\frac{3+\gamma}{1-\gamma} \beta^{2}-1\right|+8 d^{4}-d^{2}\left(\Delta U^{2}+2 a^{2}\left(1+\beta^{2}\right)\right)+\frac{\Delta U^{2}}{2} a^{2}\left(1+\beta^{2}\right) .
\end{aligned}
$$

The solution for $d$ can be found by solving the following cubic equation for the variable $y=d^{2}$ :

$$
y^{3}+b_{2} y^{2}+b_{1} y+b_{0}=0,
$$

where

$$
\begin{aligned}
& b_{2}=-\left(\frac{\Delta U}{2}\right)^{2}-\frac{1}{2} a^{2}\left(1+\beta^{2}\right), \\
& b_{1}=\frac{1}{16} a^{4}\left(1-\beta^{2}\right)^{2}+\frac{1}{2} a^{2}\left(\frac{\Delta U}{2}\right)^{2}\left(1+\beta^{2}\right)^{2}, \\
& b_{0}=-\frac{1}{16} a^{4}\left(\frac{\Delta U}{2}\right)^{2}\left(\frac{3+\gamma}{1-\gamma} \beta^{2}-1\right)^{2} .
\end{aligned}
$$


Among the three roots of the equation above, we take the only (positive) real root if there are two complex conjugate roots. If all the roots are real, the root that we need is the one with minimum (positive) value. (Note that the solution for $y$ must give a value of $d$ such that $Z^{ \pm}$is positive.) Once we have the solution for $y$, we take $|d|=\sqrt{y}$, and the sign of $d$ depends on the sign of the following quantity:

$$
Y=\Delta U\left(\frac{1+\gamma}{3+\gamma}-\varphi\right)
$$

We have $d \gtreqless 0$ for $Y \lesseqgtr 0$. In particular, note that if $u_{s}=u_{f}$ or $\varphi=\frac{1+\gamma}{3+\gamma}$, then $d=0, V_{\mathrm{e}}=V_{\mathrm{i}}=\bar{u}$, and we obtain explicit expressions for the eigenvalues (the expressions in (14) for the case $u_{s}=u_{f}=u$ ). Based on the inequalities (17), we have the following bounds on $d, V_{\mathrm{e}, \mathrm{i}}$, and $c_{\mathrm{e}, \mathrm{i}}$ :

$$
\begin{aligned}
& |d| \leqslant \min \left(\frac{|\Delta U|}{2}, \frac{a}{2}(1-\beta)\right), \\
& u_{\min } \leqslant V_{\mathrm{e}, \mathrm{i}} \leqslant u_{\max } \text { and } 0 \leqslant c_{\mathrm{i}} \leqslant c_{\mathrm{e}} \leqslant a+\frac{|\Delta U|}{2} .
\end{aligned}
$$

Introducing now the number

$$
N \equiv \frac{\Delta U}{a},
$$

we observe that the ratio

$$
\frac{d}{a}=z(N, \beta)
$$

depends only on $N$ and $\beta$ since $b_{2} / a^{2}, b_{1} / a^{4}, b_{0} / a^{6}$ and $\operatorname{sgn}(Y)$ depend only on $N, \beta$, and the quantities $c_{\mathrm{e}}, c_{\mathrm{i}}$ can be expressed as

$$
c_{\mathrm{e}, \mathrm{i}}=a \Gamma_{\mathrm{e}, \mathrm{i}}(N, \beta),
$$

where

$$
\Gamma_{\mathrm{e}, \mathrm{i}}(N, \beta)=\left(\frac{N^{2}}{4}-3 z^{2}+\frac{1}{2}\left(1+\beta^{2}\right) \pm\left(\frac{1}{4}\left(1-\beta^{2}\right)^{2} \pm N|z|\left|\frac{3+\gamma}{1-\gamma}-1\right|+8 z^{4}-z^{2}\left(N^{2}+2\left(1+\beta^{2}\right)\right)+\frac{N^{2}}{2}\left(1+\beta^{2}\right)\right)^{\frac{1}{2}}\right)^{\frac{1}{2}} .
$$

From (24), we obtain the bounds $|z| \leqslant \min \left(\frac{1}{2}|N|, \frac{1}{2}(1-\beta)\right) \leqslant \frac{1}{2}, \Gamma_{\mathrm{e}, \mathrm{i}} \leqslant\left(1+\frac{|N|}{2}\right)$, and note that in the first hyperbolic region we have $|N| \leqslant 2 \beta$.

\subsubsection{Eigenvectors}

The right and left eigenvectors of the matrix $A(q)(12 \mathrm{~b})$ can be written in terms of the eigenvalues $\lambda_{k}, k=1, \ldots, 4$. Let us consider here for simplicity $h>0, \varphi \neq 1$, and $\varphi \neq 0$, that is $h_{s}, h_{f}>0$. Then the right eigenvectors $r_{k}, k=1, \ldots, 4$, can be expressed as

$$
r_{k}=\left(\begin{array}{c}
1 \\
\lambda_{k} \\
\xi_{k} \\
\xi_{k} \lambda_{k}
\end{array}\right)
$$

with

$$
\xi_{k}=\frac{\left(\lambda_{k}-u_{s}\right)^{2}-g\left(h_{s}+\frac{1-\gamma}{2} h_{f}\right)}{g \frac{1+\gamma}{2} h_{s}}=\frac{g h_{f}}{\left(\lambda_{k}-u_{f}\right)^{2}-g h_{f}} .
$$

Assuming that the matrix of the right eigenvectors $R=\left(r_{1}, r_{2}, r_{3}, r_{4}\right)$ is non-singular, which in particular is true if $\left|u_{s}-u_{f}\right|<2 a \beta$ or $\left|u_{s}-u_{f}\right|>2 a$ (Proposition 3.1), the left eigenvectors $l_{k}$ of $A(q), k=1, \ldots, 4$, can be then taken as

$$
l_{k}=\frac{n_{k}}{P^{\prime}\left(\lambda_{k}\right)}, \quad n_{k}=\left(\vartheta_{s, k}\left(\lambda_{k}-2 u_{s}\right), \vartheta_{s, k}, \vartheta_{f}\left(\lambda_{k}-2 u_{f}\right), \vartheta_{f}\right),
$$

where $P(\lambda)=\operatorname{det}(\lambda I-A)$ is the characteristic polynomial of $A$ and

$$
\vartheta_{s, k}=\left(\lambda_{k}-u_{f}\right)^{2}-g h_{f}=g \frac{h_{f}}{\xi_{k}} \text { and } \vartheta_{f}=g \frac{1+\gamma}{2} h_{s} .
$$

Here we have normalized the left eigenvectors so that $l_{j} r_{k}=\delta_{j k}$. 


\section{Wave-propagation finite volume methods and Riemann solvers}

The class of numerical schemes that we consider for the approximation of the single-phase and the two-phase shallow flow models are the wave propagation methods of [36]. These are a class of finite volume schemes for the solution of hyperbolic systems based on Riemann solvers (Godunov-type schemes), cf. [37,11-13,20]. In fact, as we mentioned in Section 1, the relaxation approach that we propose results in the definition of a particular Riemann solver for the shallow flow systems (see Sections 5 and 7). For the numerical solution of the two-phase model (8) we assume that solid and fluid velocity differences are small enough so that the system is hyperbolic. Potential difficulties related to the appearance of complex eigenvalues will be mentioned in Section 7.5. Note also that here we will illustrate a method only for the solution of the homogeneous two-phase system. Inter-phase drag source terms will be taken into account in some of the numerical experiments, and they are approximated by the fractional step algorithm described in [10].

Let us consider a general hyperbolic system of the form

$$
\partial_{t} q+A(q) \partial_{x} q=0, \quad q \in \mathbb{R}^{\mu}, A \in \mathbb{R}^{\mu \times \mu} .
$$

A Riemann solver for this system of equations defines a function $q_{\mathrm{RS}}\left(x / t ; q_{\ell}, q_{\mathrm{r}}\right)$ that approximates the true similarity solution to a Riemann problem for the system with left and right data $q_{\ell}$ and $q_{r}$. For many solvers (e.g. HLL, Roe, Suliciu, ...) this function $q_{\mathrm{RS}}\left(x / t ; q_{\ell}, q_{r}\right)$ consists of a set of $M$ waves $\mathcal{W}^{k}$ and corresponding speeds $s^{k}, M \gtreqless \mu$ (e.g. $M=\mu$ for Roe, $M=2$ for HLL). That is, $\left.q_{\mathrm{RS}}\right|_{x / t<s^{1}}=q_{\ell},\left.q_{\mathrm{RS}}\right|_{x / t>s^{M}}=q_{r}$, and

$$
\left.q_{\mathrm{RS}}\left(x / t ; q_{\ell}, q_{r}\right)\right|_{s^{k}<x / t<s^{k+1}}=q_{\ell}+\sum_{j=1}^{k} \mathcal{W}^{j}=q_{r}-\sum_{j=1}^{M-k} \mathcal{W}^{M-j+1}, \quad k=1, \ldots, M-1 .
$$

The sum of the waves must be equal to the initial jump in the system variables:

$$
\Delta q \equiv q_{r}-q_{\ell}=\sum_{k=1}^{M} \mathcal{W}^{k}
$$

Moreover, for conservative systems endowed with a flux function $f(q), f^{\prime}(q)=A(q)$, i.e. systems of the form $\partial_{t} q+\partial_{x} f(q)=0$, the initial flux jump must be recovered by the sum of the waves multiplied by the corresponding speeds:

$$
\Delta f \equiv f\left(q_{r}\right)-f\left(q_{\ell}\right)=\sum_{k=1}^{M} s^{k} \mathcal{W}^{k}
$$

The quantities $\mathcal{Z}^{k}=s^{k} \mathcal{W}^{k}$ have the dimension of a flux, and we will call them $f$-waves following the nomenclature introduced in [38] (the nomenclature holds for both the conservative and the non-conservative case).

The updating formula of the corresponding Godunov-type finite volume scheme can be written in the following LeVeque's wave-propagation form [36,13] in terms of the $f$-waves $\mathcal{Z}_{i+1 / 2}^{k}$ and speeds $s_{i+1 / 2}^{k}$ arising from local Riemann problems with data $Q_{i}^{n}, Q_{i+1}^{n}(i \in \mathbb{Z}$ and $n \in \mathbb{N}$ are the indexes of the discretization in space and time, respectively):

$$
\begin{aligned}
& Q_{i}^{n+1}=Q_{i}^{n}-\frac{\Delta t}{\Delta x}\left(\mathcal{A}^{+} \Delta Q_{i-1 / 2}+\mathcal{A}^{-} \Delta Q_{i+1 / 2}\right)-\frac{\Delta t}{\Delta x}\left(F_{i+1 / 2}^{c}-F_{i-1 / 2}^{c}\right), \\
& F_{i+1 / 2}^{c}=\frac{1}{2} \sum_{k=1}^{M} \operatorname{sgn}\left(s_{i+1 / 2}^{k}\right)\left(1-\frac{\Delta t}{\Delta x}\left|s_{i+1 / 2}^{k}\right|\right) \mathcal{Z}_{i+1 / 2}^{c, k},
\end{aligned}
$$

where $\mathcal{A}^{\mp} \Delta Q$ are the fluctuations at cell interfaces,

$$
\mathcal{A}^{-} \Delta Q_{i+1 / 2}=\sum_{k: s_{i+1 / 2}^{k}<0} \mathcal{Z}_{i+1 / 2}^{k} \text { and } \mathcal{A}^{+} \Delta Q_{i+1 / 2}=\sum_{k: s_{i+1 / 2}^{k}>0} \mathcal{Z}_{i+1 / 2}^{k},
$$

and $F_{i+1 / 2}^{c}$ are correction fluxes for second order resolution. $\mathcal{Z}_{i+1 / 2}^{c, k}$ are a modified version of $\mathcal{Z}_{i+1 / 2}^{k}$, obtained by applying to $\mathcal{Z}_{i+1 / 2}^{k}$ a limiter function, cf. [13].

\subsection{Roe and Roe-type Riemann solvers}

Classical Roe's Riemann solver [34] for systems of conservation laws, and Roe-type solvers for more general systems of the form (30), are based on a linearization of the system's equations. These solvers approximate the solution to a Riemann problem for the original system with initial data $q_{\ell}, q_{r}$ by the exact solution of a Riemann problem for a linearized system

$$
\partial_{t} q+\widehat{A}\left(q_{\ell}, q_{r}\right) \partial_{x} q=0 .
$$

The constant coefficient matrix $\widehat{A}\left(q_{\ell}, q_{r}\right)$ (Roe matrix) must guarantee conservation for the variables of the model system that are governed by conservative equations. That is, if the $k$ th component $q^{(k)}$ of the vector $q$ is a conserved quantity, and $f^{(k)}$ denotes the associated flux function, then we need 


$$
\widehat{A}_{(k,:)}\left(q_{r}-q_{\ell}\right)=f^{(k)}\left(q_{r}\right)-f^{(k)}\left(q_{\ell}\right),
$$

where $A_{(k,:)}$ is the $k$ th row of the matrix $\widehat{A}$. We refer the reader to [11,37] for a rigorous definition of Roe matrix and to [39-41] for a generalized definition of Roe linearization based on the use of a family of paths.

For the conservative shallow flow system (1) the Roe matrix is classically defined as the original matrix $A(q)(3 \mathrm{~b})$ evaluated at an average state $\hat{q}=\hat{q}(\hat{h}, \hat{u})$, where

$$
\hat{h}=\frac{h_{\ell}+h_{r}}{2} \text { and } \hat{u}=\frac{\sqrt{h_{\ell}} u_{\ell}+\sqrt{h_{r}} u_{r}}{\sqrt{h_{\ell}}+\sqrt{h_{r}}} .
$$

In $[9,10]$ a Roe-type Riemann solver was presented for the solution of the non-conservative two-phase flow model (12). Here the constant coefficient matrix $\widehat{A}\left(q_{\ell}, q_{r}\right)$ must guarantee conservation for the mass of each phase and for the momentum of the mixture, that is (36) for $k=1$ and $k=3$, and

$$
\left(\widehat{A}_{(2,:)}+\gamma \widehat{A}_{(4,:)}\right)\left(q_{r}-q_{\ell}\right)=f_{\mathrm{m}}\left(q_{r}\right)-f_{\mathrm{m}}\left(q_{\ell}\right) .
$$

This can be obtained by taking $\widehat{A}$ as the matrix $A(q)$ in (12b) evaluated at an average state $\hat{q}=\hat{q}\left(\hat{h}_{s}, \hat{h}_{f}, \hat{u}_{s}, \hat{u}_{f}\right)$, where

$$
\hat{h}_{\theta}=\frac{h_{\theta, \ell}+h_{\theta, r}}{2} \quad \text { and } \quad \hat{u}_{\theta}=\frac{\sqrt{h_{\theta, \ell}} u_{\theta, \ell}+\sqrt{h_{\theta, r}} u_{\theta, r}}{\sqrt{h_{\theta, \ell}}+\sqrt{h_{\theta, r}}}, \quad \theta=s, f .
$$

Resulting waves and speeds for Roe and Roe-type solvers are defined by $(M=\mu)$

$$
\mathcal{W}^{k}=\hat{\alpha}_{k} \hat{r}_{k} \quad \text { and } \quad s^{k}=\hat{\lambda}_{k}, \quad k=1, \ldots, \mu,
$$

where $\left\{\hat{r}_{k}, \hat{\lambda}_{k}\right\}_{1 \leqslant k \leqslant \mu}$ are the eigenpairs of the Roe matrix $\widehat{A}$ of the considered system, and $\hat{\alpha}_{k}$ are the coefficients of the eigendecomposition $\Delta q=\sum_{k=1}^{\mu} \hat{\alpha}_{k} \hat{r}_{k}$. Hence, $\hat{\alpha}_{k}=\hat{l}_{k} \Delta q$, where $\hat{l}_{k}$ are the left eigenvectors of $\widehat{A}$, with the normalization $\hat{l}_{j} \hat{r}_{k}=\delta_{j k}$. The $f$-waves to be used in (34c) are then

$$
\mathcal{Z}^{k}=\hat{\lambda}_{k} \mathcal{W}^{k}=\hat{\lambda}_{k} \hat{\alpha}_{k} \hat{r}_{k}
$$

\subsection{The problem of positivity preservation}

A well-known drawback of numerical schemes based on Roe and Roe-type solvers is that they may generate negative discrete values of physically non-negative variables, the flow height and phase volume fractions in the models under study. The difficulties related to positivity ${ }^{2}$ preservation of Godunov-type schemes were analyzed in detail by Einfeldt-Munz-RoeSjögreen [42] in the context of the Euler equations of gas dynamics. In [42] the authors introduced the notion of positively conservative scheme, which here we state in a general form:

Definition 4.1. A numerical scheme for the approximation of a given system of equations endowed with a set $\Omega$ of physically admissible states is positivity preserving if, for any choice of the initial data in $\Omega$, the computed values of the solution belong to $\Omega$ at any discrete spatial location and at any time level.

For the models (1) and (8) physical consistency requires that the discrete solution values belong to the set of admissible states (4) and (13a), respectively. Hence we need

$$
h_{i}^{n} \geqslant 0, \quad \forall i \in \mathbb{Z}, \forall n \in \mathbb{N},
$$

for the single-phase model, and

$$
h_{i}^{n} \geqslant 0, \quad \varphi_{i}^{n} \in[0,1] \Longleftrightarrow h_{s, i}^{n}, h_{f, i}^{n} \geqslant 0, \quad \forall i \in \mathbb{Z}, \forall n \in \mathbb{N},
$$

for the two-phase model.

Let us consider the particular class of Godunov-type schemes in the sense of Harten-Lax-van Leer [17]. For an approximation $q_{\mathrm{RS}}\left(x / t ; q_{\ell}, q_{r}\right)$ to local Riemann problems, and under suitable consistency conditions, such schemes are defined by [17, Theorem 3.1]

$$
Q_{i}^{n+1}=\frac{1}{\Delta x} \int_{0}^{\Delta x / 2} q_{\mathrm{RS}}\left(x / t ; Q_{i-1}^{n}, Q_{i}^{n}\right) d x+\frac{1}{\Delta x} \int_{-\Delta x / 2}^{0} q_{\mathrm{RS}}\left(x / t ; Q_{i}^{n}, Q_{i+1}^{n}\right) d x,
$$

that is the discrete solution is computed (at first order) by cell $L^{2}$-averaging of the local approximate Riemann solutions. Roe an Roe-type solvers, HLL solvers, and relaxation solvers in the sense of Bouchut [20] enter in this class of methods, c.f. [20] for a rigorous and detailed presentation.

\footnotetext{
${ }^{2}$ The term positivity is classically used in this context in the literature instead of the more precise term non-negativity. We will use indifferently the two terms.
} 
For these Godunov-type schemes a sufficient condition for positivity is non-negativity of the approximate Riemann solution for the physically non-negative variables, as we enunciate below.

Lemma 4.2. A Godunov-type scheme in the sense of Harten-Lax-van Leer (44) for a given system of equations endowed with a convex set $\Omega$ of physically admissible states is positivity preserving if the Riemann solution states generated by the associated Riemann solver belong to $\Omega$ for any choice of the initial Riemann data in $\Omega$, and under the condition $\mathrm{CFL} \leqslant 1 / 2$, where

$$
\mathrm{CFL}=\frac{\Delta t}{\Delta x} \max _{i}\left(\left|s_{i+1 / 2}^{k}\right|, k=1, \ldots, M\right) .
$$

Proof. The proof was given in [42]. Assume $q_{\mathrm{RS}}\left(x / t ; q_{\ell}, q_{r}\right) \in \Omega$, for any pair $q_{\ell}, q_{r} \in \Omega$ and CFL $\leqslant 1 / 2$. Then the computed solution values $Q_{i}^{n+1}$ belong to $\Omega, \forall i \in \mathbb{Z}$ and $\forall n \in \mathbb{N}$, since they are obtained by convex averaging of Riemann solution states that belong to the convex set $\Omega$.

The Roe Riemann solution structure for the single-phase shallow flow model (1) consists of three constant states $q_{\ell}, q_{\mathrm{Roe}}^{*}, q_{r}$, separated by two linear waves propagating at speeds

$$
\hat{\lambda}_{1,2}=\hat{u} \mp \hat{c}, \quad \text { where } \hat{c}=\sqrt{g \hat{h}}
$$

with the definitions (37) of $\hat{h}, \hat{u}$. By using the Roe waves (40), and the identity

$$
\Delta m=\hat{u} \Delta h+\sqrt{h_{\ell} h_{r}} \Delta u,
$$

where $\Delta(\cdot) \equiv(\cdot)_{r}-(\cdot)_{\ell}$, the flow height corresponding to the intermediate state $q_{\mathrm{Roe}}^{*}$ can be easily found as

$$
h_{\mathrm{Roe}}^{*}=\frac{h_{\ell}+h_{\mathrm{r}}}{2}-\frac{\sqrt{h_{\ell} h_{r}} \Delta u}{2 \hat{c}} .
$$

For fixed initial data $h_{\ell}, h_{r}$, we see that $h_{\mathrm{Roe}}^{*}$ becomes negative for positive values of the initial velocity jump $\Delta u$ sufficiently large, $\Delta u>\frac{\hat{c}\left(h_{\ell}+h_{r}\right)}{\sqrt{h_{\ell} h_{r}}}$. This typically happens for Riemann problems consisting of two rarefactions moving in opposite directions that form a region of very low flow depth or a vacuum zone (dry bed) in between (cf. [14]; [13, p. 327]). If for a Riemann problem at some grid cell interface $h_{\mathrm{Roe}}^{*}<0$, then the numerical scheme will likely produce negative discrete values of $h$, and the numerical code will fail when computing quantities $\sqrt{g h}$. Let us also recall that failure of the standard Roe solver near vacuum states for shallow water equations as well as for Euler equations can be imputed to an underestimation of the physical signal velocities by the numerical signal velocities (the Roe speeds (46)). See the discussion of EinfeldtMunz-Roe-Sjögreen in [42, p. 285] for the Euler equations. Unfortunately, the conservation constraints on the definition of the Roe matrix $\widehat{A}=A(\hat{q})$ prevent from using a choice of the average state $\hat{q}$ that could allow enlarging suitably the Roe velocities to ensure positivity of the numerical scheme.

Clearly, similar numerical difficulties for positivity preservation are encountered by the Roe-type method for the twophase model. In this case negative values of $h_{s}$ and/or $h_{f}$ may be produced.

In the following we present a Riemann solver based on a relaxation model that has additional degrees of freedom with respect to Roe and Roe-type solvers. This new solver guarantees conservation for any choice of the relaxation parameters, which can be freely set to obtain numerical speeds that ensure robustness of the method near dry states.

Let us mention that for the single-phase shallow flow system a positivity preserving Roe method can be obtained by defining a Roe matrix in a new form that differs from the classical choice of the average Jacobian $\widehat{A}=A(\hat{q})$. This approach is described in Appendix A, where we also discuss why such strategy unfortunately does not seem applicable to the twophase shallow flow system.

\section{Relaxation method for the single-phase shallow flow model}

We derive in this section an approximate Riemann solver for the single-phase shallow flow equations (1) by introducing a relaxation model for the system. Our approach was in particular suggested by the recent relaxation solver of Berthon and Marche [21], but the main ideas follow primarily the work of Jin and Xin [29] and Suliciu [18,19]. Other related works are for instance $[30,31,23]$. We refer in particular to the monograph $[20]$ and the bibliography therein. We introduce an auxiliary variable $\omega$ that is meant to be an approximation of the momentum $m$, and approaches $m$ as a relaxation time $\tau \rightarrow 0^{+}$. This relaxation variable $\omega$ is used to replace the momentum variable $m$ in the spatial gradients of the original system. We propose that $\omega$ is governed by a linearized form of the momentum equation:

$$
\partial_{t} \omega+\left(-\tilde{u}^{2}+g \tilde{h}\right) \partial_{x} h+2 \tilde{u} \partial_{x} \omega=\frac{m-\omega}{\tau},
$$

where the linearization has been considered at an average state $\tilde{q}(\tilde{h}, \tilde{u})$, and the source term on the right-hand side drives the relaxation process to equilibrium. Then the relaxation system has the form: 


$$
\begin{aligned}
& \partial_{t} h+\partial_{x} \omega=0, \\
& \partial_{t} m+\partial_{x}\left(\frac{\omega^{2}}{h}+\frac{g}{2} h^{2}\right)=0, \\
& \partial_{t} \omega+\left(-\tilde{u}^{2}+g \tilde{h}\right) \partial_{x} h+2 \tilde{u} \partial_{x} \omega=\frac{m-\omega}{\tau} .
\end{aligned}
$$

Formally, the system above recovers the original system in the limit $\tau \rightarrow 0^{+}$(equilibrium limit).

Here we define a solution method for the shallow flow system (1) that is based on the relaxed scheme of Jin-Xin [29]. The algorithm consists of two alternating steps:

1. Set the relaxation variable $\omega$ equal to its equilibrium value $m$ in the initialization of the data at time $t^{n}$ (equilibrium step);

2. Solve exactly over a time step local Riemann problems at cell interfaces for the relaxation system with no relaxation source term to update $(h, m)^{n}$ to $(h, m)^{n+1}$ (propagation step).

Since we reset $\omega^{n}=m^{n}$ at each time level, note that the solution for $\omega$ coming from the propagation step is ignored.

When the algorithm above is used, the Riemann solution of the relaxation system results in the definition of an approximate Riemann solution $q_{\mathrm{RS}}^{\mathrm{rel}}\left(x / t ; q_{\ell}, q_{r}\right)$ for the original system, see [23,20]. The resulting numerical approximation method for (1) is a Godunov-type scheme in the sense of Harten-Lax-van Leer (44) associated to a function $q_{\mathrm{RS}}^{\text {rel }}\left(x / t ; q_{\ell}, q_{r}\right)$ [20]. Because we will not be concerned with the relaxation source term, hereafter we will intend as relaxation model simply the homogeneous relaxation system, which, denoting $q^{\mathrm{E}}=(h, m, \omega)^{\mathrm{T}}$, has the quasi-linear form:

$$
\partial_{t} q^{\mathrm{E}}+A^{\mathrm{E}}\left(q^{\mathrm{E}}\right) \partial_{x} q^{\mathrm{E}}=0
$$

with

$$
A^{\mathrm{E}}\left(q^{\mathrm{E}}\right)=\left(\begin{array}{ccc}
0 & 0 & 1 \\
-\frac{\omega^{2}}{h}+g h & 0 & 2 \frac{\omega}{h} \\
-\tilde{u}^{2}+g \tilde{h} & 0 & 2 \tilde{u}
\end{array}\right) .
$$

\subsection{Riemann structure of the relaxation model}

Let us note that the relaxation model (51) presents a decoupled sub-system for the variables

$$
q^{\mathrm{R}} \equiv\left(\begin{array}{c}
h \\
\omega
\end{array}\right) .
$$

This sub-system corresponds to a linearized form of the original system (3),

$$
\partial_{t} q^{\mathrm{R}}+\widetilde{A} \partial_{x} q^{\mathrm{R}}=0
$$

with a matrix $\widetilde{A}=A(\tilde{q})$, where $A(q) \in \mathbb{R}^{2 \times 2}$ is the matrix (3b), and $\tilde{q}=\tilde{q}(\tilde{h}, \tilde{u})$. The eigenvalues of the relaxation model are the two eigenvalues of this sub-system, that is the eigenvalues of $\widetilde{A}$, plus a zero eigenvalue:

$$
\tilde{\lambda}_{1,2}=\tilde{u} \mp \tilde{c}, \quad \text { where } \tilde{c}=\sqrt{g \tilde{h}} \text {, and } \lambda^{0}=0 .
$$

The eigenvectors corresponding to $\tilde{\lambda}_{1,2}$ are

$$
r_{1,2}^{\mathrm{E}}=\left(\begin{array}{c}
1 \\
\frac{2 \omega}{h}-\frac{\omega^{2} / h^{2}-g h}{\tilde{u} \mp \tilde{c}} \\
\tilde{u} \mp \tilde{c}
\end{array}\right),
$$

and the eigenvector associated to $\lambda^{0}=0$ is $r^{\mathrm{E} 0}=(0,1,0)^{\mathrm{T}}$. Note that all the characteristic fields are linearly degenerate. The Riemann solution of the relaxation system consists then of constant states separated by linear waves (contact discontinuities), a stationary wave corresponding to $\lambda^{0}$, and two waves propagating at speeds $\tilde{\lambda}_{1,2}$.

\subsubsection{Riemann invariants}

The variables $q^{\mathrm{R}}=(h, \omega)^{\mathrm{T}}$ are Riemann invariants across the stationary discontinuity $\lambda^{0}$ :

$$
\Delta^{0} h=0 \text { and } \Delta^{0} \omega=0,
$$

where $\Delta^{0}(\cdot)$ denotes increments across $\lambda^{0}$. Therefore, only the momentum variable $m$ has possibly a jump across this stationary wave. Across the two propagating waves associated to $\tilde{\lambda}_{1,2}=\tilde{u} \mp \tilde{c}$, the following invariance relations hold: 


$$
\begin{aligned}
& \tilde{\lambda}_{k} h-\omega=\text { const. and } \\
& \tilde{\lambda}_{k} m-\mathcal{F}(h, \omega)=\text { const., where } \mathcal{F}(h, \omega)=\frac{\omega^{2}}{h}+\frac{g}{2} h^{2} .
\end{aligned}
$$

Denoting with $\Delta_{k}(\cdot)$ increments across the waves $\tilde{\lambda}_{k}, k=1,2$, we have therefore the following jump relations for these waves:

$$
\begin{aligned}
& \tilde{\lambda}_{k} \Delta_{k} h=\Delta_{k} \omega, \\
& \tilde{\lambda}_{k} \Delta_{k} m=\Delta_{k} \mathcal{F}(h, \omega),
\end{aligned}
$$

which can be also written as

$$
\tilde{\lambda}_{k} \Delta_{k} q=\Delta_{k} f\left(q^{\mathrm{R}}\right) \text {, }
$$

where $f(q)$ is the flux vector of the shallow flow system (2). Note that the relations (59) have the form of Rankine-Hugoniot jump conditions.

\subsection{Relaxation Riemann solver}

The exact solution $q_{\mathrm{RS}}^{\mathrm{E}}\left(x / t ; q_{\ell}^{\mathrm{E}}, q_{r}^{\mathrm{E}}\right) \in \mathbb{R}^{3}$ to a Riemann problem for the relaxation system (51) with left and right data $q_{\ell}^{\mathrm{E}}, q_{r}^{\mathrm{E}}$ defines an approximate Riemann solution $q_{\mathrm{RS}}^{\text {rel }}\left(x / t ; q_{\ell}, q_{r}\right) \in \mathbb{R}^{2}$ for the original system (3) with data $q_{\ell}, q_{r}$, i.e. $\left(q_{\mathrm{RS}}^{\text {rel }}\right)^{(k)}=\left(q_{\mathrm{RS}}^{\mathrm{E}}\right)^{(k)}, k=1,2$. Notice that the equilibrium step of the relaxed scheme introduced at the beginning of this section amounts to setting the right and left data of the relaxation variables as

$$
\omega_{\ell}=m_{\ell} \text { and } \omega_{r}=m_{r} .
$$

The Riemann solution for the variables $q^{\mathrm{R}}$ of the relaxation system is the Riemann solution of the linear sub-system (53). The increments $\Delta_{k} q^{\mathrm{R}}, k=1,2$, across the (linear) waves $\tilde{\lambda}_{1,2}=\tilde{u} \mp \tilde{c}$ are:

$$
\Delta_{k} q^{\mathrm{R}}=\left(\Delta_{k} h, \Delta_{k} \omega\right)^{\mathrm{T}}=\tilde{\alpha}_{k} \tilde{r}_{k}, \quad k=1,2,
$$

where $\tilde{r}_{k}$ are the right eigenvectors of $\tilde{A}, \tilde{r}_{1,2}=(1, \tilde{u} \mp \tilde{c})^{\mathrm{T}}$, and $\tilde{\alpha}_{k}$ are the coefficients of the projection $\Delta q^{\mathrm{R}}=\sum_{k=1}^{2} \tilde{\alpha}_{k} \tilde{r}_{k}($ here $\Delta(\cdot) \equiv(\cdot)_{r}-(\cdot)_{\ell}$, as in Section 4.2). We have $\tilde{\alpha}_{k}=\tilde{l}_{k} \Delta q^{\mathrm{R}}$, where $\tilde{l}_{k}$ are the left eigenvectors of $\tilde{A}, \tilde{l}_{1,2}=\frac{1}{2 \tilde{c}}( \pm \tilde{u}+\tilde{c}, \mp 1)$. The intermediate state $\left(q^{\mathrm{R}}\right)^{*}=\left(\boldsymbol{h}^{*}, \omega^{*}\right)$ between the waves $\tilde{\lambda}_{1}$ and $\tilde{\lambda}_{2}$ can be then found as

$$
\left(q^{\mathrm{R}}\right)^{*}=q_{\ell}^{\mathrm{R}}+\tilde{\alpha}_{1} \tilde{r}_{1}=q_{r}^{\mathrm{R}}-\tilde{\alpha}_{2} \tilde{r}_{2} .
$$

Clearly, $\left(q^{\mathrm{R}}\right)^{*}$ and $\Delta_{k} q^{\mathrm{R}}$ can be equivalently obtained through the Riemann invariants (57a). In particular, note that we have the equality $\tilde{\lambda}_{k} \Delta_{k} h=\Delta_{k} \omega=\tilde{\lambda}_{k} \tilde{\alpha}_{k}$.

The increments for the momentum $m$ across the two propagating waves are then given by the jump relations (58b), which correspond to the Riemann invariants (57b). Finally, the jump of the momentum across $\lambda^{0}$ is $\Delta^{0} m=\Delta m-\sum_{k=1}^{2} \Delta_{k} m$, and the Riemann solution intermediate states for $m$ can be found by distinguishing between the different cases corresponding to a different order of $\tilde{\lambda}_{1}, \tilde{\lambda}_{2}$ with respect to $\lambda^{0}$, however this information is not needed in the solver (see below). The resulting approximate Riemann solver for the original system consists of three waves

$$
\mathcal{W}^{k}=\Delta_{k} q, \quad k=1,2, \quad \text { and } \mathcal{W}^{3}=\left(0, \Delta^{0} m\right)^{\mathrm{T}},
$$

moving at speeds $s^{k}$ given by

$$
s^{1,2}=\tilde{\lambda}_{1,2}=\tilde{u} \mp \tilde{c} \text { and } s^{3}=\lambda^{0}=0 .
$$

The wave structure can be written in terms of the $f$-waves $\mathcal{Z}^{k}=s^{k} \mathcal{W}^{k}$. We have

$$
\mathcal{Z}^{k}=\tilde{\lambda}_{k} \Delta_{k} q, \quad k=1,2, \quad \text { and } \quad \mathcal{Z}^{3}=0,
$$

where $\tilde{\lambda}_{k} \Delta_{k} q$ are obtained as explained above. We rewrite here for clarity the resulting formulas:

$$
\begin{aligned}
& \tilde{\lambda}_{k} \Delta_{k} h=\tilde{\lambda}_{k} \tilde{\alpha}_{k}, \\
& \tilde{\lambda}_{k} \Delta_{k} m=\Delta_{k}\left(\frac{\omega^{2}}{h}+\frac{g}{2} h^{2}\right), \quad k=1,2 .
\end{aligned}
$$

Note that in the wave propagation algorithm (34) we only need to specify $f$-waves and speeds, and not the waves $\mathcal{W}^{k}$ themselves. This avoids computing the jump of the momentum across the eigenvalue $\lambda^{0}=0$. It is easy to see that the solver is conservative for any choice of $\tilde{q}$, since, based on (59) and (60),

$$
\sum_{k=1}^{3} \mathcal{Z}^{k}=\Delta f(q) \text {. }
$$

Let us observe that the relaxation procedure has led to a Riemann solver that uses a Riemann solution $q^{\mathrm{R}}$ formally computed as the Roe Riemann solution, the difference possibly being in the definition of the averages $(\cdot \hat{)},(\tilde{)})$ of $h, u$. This is obvious by looking at the linearized problems (53) and (35), and it has motivated our choice of the superscript R of $q^{\mathrm{R}}$, which stands 
for "Roe". Then, the f-waves of the relaxation solver are defined as the flux jumps corresponding to this Roe Riemann solution, see (65) and (59), whereas the standard Roe solver uses the eigencomponents of the linearized system. Furthermore, as a consequence of the linearity of the first component of the flux function $f(q)$ of the model system, the flow height wave components and the flow height Riemann solution are formally computed in the same way by the relaxation and Roe solvers (cf. (66a) and (41)). Then the two methods differ only in the expression of the momentum wave components. Based on this observations, the relaxation solver can be seen as a modified Roe solver that allows conservation for arbitrary choices of the parameters by a new definition of the momentum waves. Indeed such a solver for the shallow water equations is not entirely a novelty, as its wave structure results to be equivalent to that of the VFRoe solver with conservative variables of Gallouët and co-workers of [32,33], as we explain in Section 6.

Let us also finally mention that, like Roe and VFRoe solvers, our relaxation solver may fail when the linearized problem's eigenvalues are close to sonic points, and an entropy fix is needed. In particular, we use the Harten and Hyman entropy-fix correction [43], in the LeVeque form described in [37] (see also [14]).

\subsection{Relaxation parameters and positivity}

We now exploit the freedom in the choice of the parameters $\tilde{h}, \tilde{u}$ to ensure positivity of the Riemann solution of the relaxation system, which is a sufficient condition for positivity of the numerical scheme, by Lemma 4.2. We have seen that there is only one intermediate Riemann state $h^{*}$ for the flow height, as a result of the invariance property in (56). Then, for positivity, it suffices to satisfy

$$
h^{*} \geqslant 0
$$

Motivated by the interpretation of the relaxation solver as a correction of the Roe solver, here we use in general the Roe speeds (46) as relaxation speeds (64), and we possibly modify them only when locally needed to enforce (68). In fact, far from vacuum regions, the Roe's speeds prove to be an efficient, stable, and low-diffusivity choice. Note that with this choice the relaxation solver possesses the Roe's solver property of exactly resolving single shocks, based on (59) and the equality $\widehat{A} \Delta q^{\mathrm{R}}=\Delta f\left(q^{\mathrm{R}}\right)$ (and let us recall, following Toro [14, p. 110], that a shock wave cannot be adjacent to a region of dry bed).

Making a step further, we propose to keep the average relaxation velocity $\tilde{u}$ equal to the Roe velocity for any Riemann problem:

$$
\tilde{u} \equiv \hat{u}=\frac{\sqrt{h_{\ell}} u_{\ell}+\sqrt{h_{r}} u_{r}}{\sqrt{h_{\ell}}+\sqrt{h_{r}}} .
$$

Then, we look for a definition of $\tilde{h}$ that fulfills (68), and such that

$$
\tilde{c}=\sqrt{g \tilde{h}} \geqslant \sqrt{g \hat{h}}
$$

or, equivalently,

$$
\tilde{h} \geqslant \hat{h} \text {. }
$$

This stability constraint means that the propagation speeds of the relaxation solver are at least as large as the propagation speeds of the Roe solver. By using (62) and the identity (47), the positivity condition (68) reads

$$
h^{*}=\frac{h_{\ell}+h_{r}}{2}-\frac{\sqrt{h_{\ell} h_{r}} \Delta u}{2 \tilde{c}} \geqslant 0,
$$

and this gives the condition on $\tilde{h}$ :

$$
\tilde{h} \geqslant \frac{h_{\ell} h_{r}(\Delta u)_{+}^{2}}{g\left(h_{\ell}+h_{r}\right)^{2}}, \quad(\cdot)_{+} \equiv \text { positive part. }
$$

We can satisfy (73) and (71) by simply defining

$$
\tilde{h} \equiv \max \left(\hat{h}, \frac{h_{\ell} h_{r}(\Delta u)_{+}^{2}}{g\left(h_{\ell}+h_{r}\right)^{2}}\right) .
$$

This choice implies that $\tilde{h}>\hat{h}$ only when the Roe average $\hat{h}$ gives a negative value of the intermediate state $h^{*}$. Based on Lemma 4.2 , and by construction of $\widetilde{A}$, we can finally state the result below.

Theorem 5.1. The first-order wave-propagation scheme for the single-phase shallow flow system (1) that uses the relaxation Riemann solver defined by (64)-(66) is positivity preserving with the definition $\widetilde{A}=A(\tilde{q}(\tilde{h}, \tilde{u})), \tilde{h}, \tilde{u}$ as in (74), (69), and under the condition $\mathrm{CFL} \leqslant 1 / 2$. 
To guarantee positivity of the second-order wave propagation scheme (34) we follow the simple approach proposed in [44], which consists in re-limiting the correction fluxes $F_{i+1 / 2}^{c}$ if they drive $h$ negative in one cell. This means that the method is accurate only at first order near wet/dry transitions (and shocks). Let us finally remark that although positivity is rigorously demonstrated only for $\mathrm{CFL} \leqslant 1 / 2$, practically the method proves to preserve positivity for CFL close to 1 (see Section 8).

\subsection{Roe-relaxation method}

A more efficient implementation of the numerical scheme consists in using a hybrid Roe-relaxation method that uses the momentum waves given by the Roe solver if (74) gives $\tilde{h}=\hat{h}$, and the momentum waves given by the relaxation solver otherwise. That is, if $\hat{h} \geqslant \frac{h_{\ell} h_{r}(\Delta u)_{t}^{2}}{g\left(h_{\ell}+h_{r}\right)^{2}}$, instead of (66b) we simply employ the momentum $f$-wave components given by Roe's method (Eq. (41)):

$$
\tilde{\lambda}_{k} \Delta_{k} m=\tilde{\lambda}_{k}\left(\tilde{\lambda}_{k} \tilde{\alpha}_{k}\right), \quad k=1,2 .
$$

In fact, if the relaxation parameters are equal to the Roe averages, the increments $\Delta_{k} q^{\mathrm{R}}$ in (61) suffice for updating the solution through Roe's formulas. Then we avoid computing the momentum increments (66b) given by the relaxation solver, which require the computation of the intermediate state $\left(q^{\mathrm{R}}\right)^{*}=\left(h^{*}, \omega^{*}\right)$. Let us remark that, although the Roe method and the relaxation method give analogous results far from vacuum states, the entropy fix technique [37] proves to be more effective for the Roe's solver, which then handles more efficiently transonic rarefactions. This is related to the discontinuity of the momentum variable in the relaxation Riemann solution across $\lambda^{0}=0$. Therefore the adaptive use of the relaxation solver performed by the hybrid method appears ultimately as the most efficient solution technique.

\section{Equivalence with the VFRoe solver}

In analogy with the work of Berthon and Marche [21], the relaxation method that we have introduced results to be equivalent to a variant of the VFRoe scheme. In [21] the authors identify the VFRoe-ncv solver with non-conservative variables $(2 c, u)[26-28]$ with a relaxation solver that is obtained by introducing two auxiliary variables approximating $c$ and $u$. The choice of this pair of variables is related to the Riemann invariants of the single-phase system, $u \pm 2 \sqrt{g h}$.

In the present work we find equivalence with the first original version of the VFRoe method [32,33], which uses conservative variables $(h, m)$. This VFRoe scheme is an approximate Godunov-type scheme applicable to conservative systems $\partial_{t} q+\partial_{x} f(q)=0$. It uses the exact Riemann solution $q_{R S}^{\operatorname{lin}}\left(x / t ; q_{\ell}, q_{r}\right)$ of a Roe-type linearized system

$$
\partial_{t} q+\widetilde{A}_{\mathrm{VFR}}\left(q_{\ell}, q_{r}\right) \partial_{x} q=0
$$

Then it defines the numerical flux at interfaces $F_{\mathrm{VFR}}\left(q_{\ell}, q_{r}\right)$ as the physical flux $f(q)$ computed at interface solutions of the linearized Riemann problem (a similar idea was also mentioned earlier by LeVeque in [37, p. 146]):

$$
F_{\mathrm{VFR}}\left(q_{\ell}, q_{r}\right)=f\left(q_{\mathrm{RS}}^{\operatorname{lin}}\left(0 ; q_{\ell}, q_{r}\right)\right) \text {. }
$$

The first order updating formula of the scheme has the standard form

$$
Q_{i}^{n+1}=Q_{i}^{n}-\frac{\Delta t}{\Delta x}\left(F_{\mathrm{VFR}}\left(Q_{i}^{n}, Q_{i+1}^{n}\right)-F_{\mathrm{VFR}}\left(Q_{i-1}^{n}, Q_{i}^{n}\right)\right) .
$$

Note that the definition (77) ensures conservation for any linearization matrix $\widetilde{A}_{\mathrm{VFR}}\left(q_{\ell}, q_{r}\right)$, in contrast with the classical Roe solver.

The equivalence of our method with the VFRoe method applied to (2), provided that we use the same linearization matrix $\widetilde{A}_{\text {VFR }}=\widetilde{A}$ in (76) and (53), follows from the remarks at the end of the previous sub-section. To show this equivalence more precisely, let us note that the VFRoe scheme can be written in the wave propagation form $(34)\left(\right.$ with $\left.F_{i+1 / 2}^{c}=0\right)$ by writing

$$
F_{\mathrm{VFR}}\left(Q_{i}^{n}, Q_{i+1}^{n}\right)-F_{\mathrm{VFR}}\left(Q_{i-1}^{n}, Q_{i}^{n}\right)=\mathcal{A}^{+} \Delta Q_{i-1 / 2}^{\mathrm{VFR}}+\mathcal{A}^{-} \Delta Q_{i+1 / 2}^{\mathrm{VFR}}
$$

with the fluctuations $\mathcal{A}^{\mp} \Delta Q^{\mathrm{VFR}}$ defined as

$$
\mathcal{A}^{-} \Delta Q_{i+1 / 2}^{\mathrm{VFR}}=F_{\mathrm{VFR}}\left(Q_{i}, Q_{i+1}\right)-f\left(Q_{i}\right) \quad \text { and } \quad \mathcal{A}^{+} \Delta Q_{i+1 / 2}^{\mathrm{VFR}}=f\left(Q_{i+1}\right)-F_{\mathrm{VFR}}\left(Q_{i}, Q_{i+1}\right) .
$$

Under the assumption $\widetilde{A}_{\text {VFR }}=\widetilde{A}$, it is easy to verify the equivalence of these expressions with the fluctuations defined by the relaxation scheme. Let us consider for instance $\mathcal{A}^{-} \Delta Q_{i+1 / 2}^{\mathrm{VFR}}$. We have

$$
\mathcal{A}^{-} \Delta Q_{i+1 / 2}^{\mathrm{VFR}}= \begin{cases}f\left(Q_{i+1}\right)-f\left(Q_{i}\right) & \text { if } \tilde{\lambda}_{2, i+1 / 2}<0, \\ f\left(q_{\mathrm{VFR}}^{*}\right)-f\left(Q_{i}\right) & \text { if } \tilde{\lambda}_{1, i+1 / 2}<0<\tilde{\lambda}_{2, i+1 / 2}, \\ 0 & \text { if } \tilde{\lambda}_{1, i+1 / 2}>0,\end{cases}
$$

where $q_{\mathrm{VFR}}^{*}$ is the intermediate state between the two propagating waves $\tilde{\lambda}_{1, i+1 / 2}$ and $\tilde{\lambda}_{2, i+1 / 2}$, as given by the Riemann solution of (76). For the relaxation solver: 


$$
\mathcal{A}^{-} \Delta Q_{i+1 / 2}= \begin{cases}\mathcal{Z}_{i+1 / 2}^{1}+\mathcal{Z}_{i+1 / 2}^{2}=f\left(Q_{i+1}\right)-f\left(Q_{i}\right) & \text { if } \tilde{\lambda}_{2, i+1 / 2}<0, \\ \mathcal{Z}_{i+1 / 2}^{1}=\left(\Delta_{1} \omega, \Delta_{1} \mathcal{F}\right)_{i+1 / 2}^{\mathrm{T}}=f\left(\left(q^{\mathrm{R}}\right)^{*}\right)-f\left(Q_{i}\right) & \text { if } \tilde{\lambda}_{1, i+1 / 2}<0<\tilde{\lambda}_{2, i+1 / 2}, \\ 0 & \text { if } \tilde{\lambda}_{1, i+1 / 2}>0,\end{cases}
$$

which is clearly equivalent to (81) since, if $\widetilde{A}_{\mathrm{VFR}}=\widetilde{A}$, systems (76) and (53) have identical Riemann solution, and hence $\left(q^{\mathrm{R}}\right)^{*}=q_{\mathrm{VFR}}^{*}$. An analogous proof can be written for $\mathcal{A}^{+} \Delta Q_{i-1 / 2}^{\mathrm{VFR}}$.

More generally, the VFRoe method for a conservative system $\partial_{t} q+\partial_{x} f(q)=0$ can be interpreted as a relaxation method that uses a vector of auxiliary variables $\Psi$ approximating $q$, and the following relaxation system:

$$
\begin{aligned}
& \partial_{t} q+\partial_{\chi} f(\Psi)=0, \\
& \partial_{t} \Psi+\widetilde{A} \partial_{x} \Psi=0,
\end{aligned}
$$

where $\widetilde{A}$ is an average of the matrix $A(q)=f^{\prime}(q)$. For systems such as (1) that include a linear mass conservation equation, we do not need an auxiliary mass variable, which would give a redundant equation in the relaxation system. Therefore for the shallow flow system it suffices to introduce one relaxation variable $\omega$ for the momentum.

One advantage of the relaxation technique is that it defines a general strategy that can be employed also for systems with non-conservative terms, to which the original VFRoe method is not applicable. Although some extensions of the VFRoe method to non-conservative systems have been proposed for some specific models $[45,46,33,27]$, there is no general VFRoe framework for non-conservative systems. In this paper we apply our relaxation technique to the non-conservative two-phase model (8). The same idea could be analogously used for other non-conservative systems, such as the two-layer shallow flow model $[47-49,40,50]$. Nonetheless, we have to remind the potential difficulties of the method related to the computation of non-conservative products, see Remark 7.1.

\subsection{Positivity of the VFRoe solver}

As in [21], an important result of the identification of the VFRoe solver with the relaxation solver is the ability to establish positivity conditions for the VFRoe method. In fact, the relaxation interpretation allows to place the VFRoe scheme in the class of Godunov-type scheme of Harten-Lax-van Leer (44) via the approximate Riemann solution $q_{\mathrm{RS}}^{\text {rel }}\left(x / t ; q_{\ell}, q_{r}\right)$ defined by the relaxation solver. This enables to use the argument of non-negativity of Riemann solution intermediate states as sufficient condition for positivity of the scheme, by Lemma 4.2. When the VFRoe method was first introduced in [32,33], it was not recognized to belong to the class of the Harten-Lax-van Leer Godunov-type schemes employing an $L^{2}$ projection step, and the intermediate states positivity condition did not seem applicable. Note that appealing to this condition does not mean that positivity can be achieved, this being subject to the existence of an average matrix $\widetilde{A}$ that ensures positivity of the Riemann solution states. We have seen that for the single-phase shallow flow equations such a matrix exists, and we can enunciate the result below, which directly follows the equivalence of the VFRoe solver with the relaxation solver and Theorem 5.1.

Corollary 6.1. The VFRoe scheme (77), (78) applied to the single-phase shallow flow system (1) is positivity preserving with the choice $\widetilde{A}_{\mathrm{VFR}}=A(\tilde{q}(\tilde{h}, \tilde{u})), \tilde{h}, \tilde{u}$ as in (74), (69), and under the condition CFL $\leqslant 1 / 2$.

An analogous positivity result can be obtained for the isentropic gas dynamics equations, which generalize system (1) to the case of a pressure function $p(h), p^{\prime}(h)>0$, in the momentum flux $\left(p(h)=\frac{g}{2} h^{2}\right.$ in (1)). Refer to system (A.1) in Appendix A. In this case $A(q)$ has the form (3b) but with the entry $(2,1)$ in the general form $A_{(2,1)}=-u^{2}+p^{\prime}(h)$. Positivity of the relaxation method generalized to this system and of the equivalent VFRoe scheme is ensured if we take $\widetilde{A}_{\mathrm{VFR}}=\widetilde{A}=A\left(\tilde{q}\left(\tilde{p}^{\prime}, \tilde{u}\right)\right)$, with $\tilde{u}=\hat{u}$, and

$$
\tilde{p}^{\prime} \equiv \max \left(\hat{p}^{\prime}, \frac{h_{\ell} h_{r}(\Delta u)_{+}^{2}}{\left(h_{\ell}+h_{r}\right)^{2}}\right), \quad \text { where } \widehat{p}^{\prime}= \begin{cases}\Delta p / \Delta h & \text { if } \Delta h \neq 0, \\ p^{\prime}\left(\frac{h_{\ell}+h_{r}}{2}\right) & \text { if } \Delta h=0 .\end{cases}
$$

We recall that in the standard VFRoe method a simple mean average is chosen for the linearization matrix $\widetilde{A}_{\text {VFR }}$, which does not guarantee positivity. The authors in [33] analyzed the solver for the isentropic gas dynamics system and showed that the method could compute interfaces with negative masses and fail. The difficulty was imprecisely admitted as a drawback of the VFRoe scheme, whereas it should have been imputed to an inappropriate choice of $\hat{A}_{\text {VFr }}$. The development of the version of the VFRoe scheme with non-conservative variables (VFRoe-ncv) $[26,51,27,28]$ was also motivated by efforts to build a robust method for flows involving dry states.

\section{Relaxation method for the two-phase granular flow model}

We now apply the relaxation method illustrated above for the single-phase shallow flow system to the two-phase granular flow model in (10). We here introduce two auxiliary variables $\omega_{s}$ and $\omega_{f}$ that are meant to be approximations of the momenta $m_{s}$ and $m_{f}$ and approach $m_{s}$ and $m_{f}$ as the relaxation time $\tau \rightarrow 0^{+}$. Similar to the single-phase case, these relaxation variables $\omega_{s}$ and $\omega_{f}$ replace the momentum variables $m_{s}$ and $m_{f}$ in the spatial gradients of the original system and are governed by linearized forms of the momentum equations: 


$$
\begin{aligned}
& \partial_{t} \omega_{s}+\left(-\tilde{u}_{s}^{2}+g \tilde{h}_{s}+g \frac{1-\gamma}{2} \tilde{h}_{f}\right) \partial_{x} h_{s}+2 \tilde{u}_{s} \partial_{x} \omega_{s}+g \frac{1+\gamma}{2} \tilde{h}_{s} \partial_{x} h_{f}=\frac{m_{s}-\omega_{s}}{\tau}, \\
& \partial_{t} \omega_{f}+g \tilde{h}_{f} \partial_{x} h_{s}+\left(-\tilde{u}_{f}^{2}+g \tilde{h}_{f}\right) \partial_{x} h_{f}+2 \tilde{u}_{f} \partial_{x} \omega_{f}=\frac{m_{f}-\omega_{f}}{\tau},
\end{aligned}
$$

where the linearization has been considered at an average state $\tilde{q}\left(\tilde{h}_{s}, \tilde{h}_{f}, \tilde{u}_{s}, \tilde{u}_{f}\right)$. Omitting hereafter the relaxation source term, the relaxation system has the form:

$$
\begin{aligned}
& \partial_{t} h_{s}+\partial_{x} \omega_{s}=0, \\
& \partial_{t} m_{s}+\partial_{x}\left(\frac{\omega_{s}^{2}}{h_{s}}+\frac{g}{2} h_{s}^{2}+g \frac{1-\gamma}{2} h_{s} h_{f}\right)+\gamma g h_{s} \partial_{x} h_{f}=0, \\
& \partial_{t} h_{f}+\partial_{x} \omega_{f}=0, \\
& \partial_{t} m_{f}+\partial_{x}\left(\frac{\omega_{f}^{2}}{h_{f}}+\frac{g}{2} h_{f}^{2}\right)+g h_{f} \partial_{x} h_{s}=0, \\
& \partial_{t} \omega_{s}+\left(-\tilde{u}_{s}^{2}+g \tilde{h}_{s}+g \frac{1-\gamma}{2} \tilde{h}_{f}\right) \partial_{x} h_{s}+2 \tilde{u}_{s} \partial_{x} \omega_{s}+g \frac{1+\gamma}{2} \tilde{h}_{s} \partial_{x} h_{f}=0, \\
& \partial_{t} \omega_{f}+g \tilde{h}_{f} \partial_{x} h_{s}+\left(-\tilde{u}_{f}^{2}+g \tilde{h}_{f}\right) \partial_{x} h_{f}+2 \tilde{u}_{f} \partial_{x} \omega_{f}=0 .
\end{aligned}
$$

\subsection{Riemann structure of the two-phase relaxation model}

The two-phase relaxation model (86) presents a decoupled sub-system for the variables

$$
q^{\mathrm{R}} \equiv\left(\begin{array}{c}
h_{s} \\
\omega_{s} \\
h_{f} \\
\omega_{f}
\end{array}\right)
$$

that corresponds to a linearized form of the original two-phase system (12),

$$
\partial_{t} q^{\mathrm{R}}+\widetilde{A} \partial_{x} q^{\mathrm{R}}=0
$$

with a matrix $\widetilde{A}=A(\tilde{q})$, where $A(q) \in \mathbb{R}^{4 \times 4}$ is the matrix $(12 \mathrm{~b})$, and $\tilde{q}=\tilde{q}\left(\tilde{h}_{s}, \tilde{h}_{f}, \tilde{u}_{s}, \tilde{u}_{f}\right)^{\mathrm{T}}$. The eigenvalues of the relaxation model are the four eigenvalues of this sub-system, that is the eigenvalues $\tilde{\lambda}_{k}=\lambda_{k}(\tilde{q}), k=1, \ldots, 4$, of $\widetilde{A}=A(\tilde{q})$, plus a zero eigenvalue with double algebraic multiplicity $\lambda^{0} \equiv \lambda_{1}^{0}=\lambda_{2}^{0}=0$. The eigenvectors associated to $\lambda^{0}$ are $r_{1}^{\mathrm{E}}=(0,1,0,0,0,0)^{\mathrm{T}}$ and $r_{2}^{\mathrm{EO}}=(0,0,0,1,0,0)^{\mathrm{T}}$, while the eigenvectors corresponding to $\tilde{\lambda}_{k}$ can be written as

$$
\tilde{r}_{k}^{\mathrm{E}}=\left(\begin{array}{c}
1 \\
\left(-\frac{\omega_{s}^{2}}{h_{s}^{2}}+g h_{s}+g \frac{1-\gamma}{2} h_{f}\right) \frac{1}{\hat{\lambda}_{k}}+2 \frac{\omega_{s}}{h_{s}}+g \frac{1+\gamma}{2} h_{s} \frac{\tilde{\xi}_{k}}{\tilde{k}_{k}} \\
\tilde{\xi}_{k} \\
g h_{f} \frac{1}{\tilde{\lambda}_{k}}+\left(-\frac{\omega_{f}^{2}}{h_{f}^{2}}+g h_{f}\right) \frac{\tilde{\xi}_{k}}{\tilde{\lambda}_{k}}+2 \frac{\omega_{f}}{h_{f}} \tilde{\xi}_{k} \\
\tilde{\lambda}_{k} \\
\tilde{\xi}_{k} \tilde{\lambda}_{k}
\end{array}\right) \text {, }
$$

where $\tilde{\xi}_{k}$ is the quantity $\xi_{k}$ defined in (28b) evaluated in $\tilde{q}$. As for the single-phase case, all the characteristic fields are linearly degenerate, and the Riemann solution of the relaxation system consists of constant states separated by linear waves, a stationary discontinuity corresponding to $\lambda^{0} \equiv \lambda_{1}^{0}=\lambda_{2}^{0}=0$, and four waves propagating at speeds $\tilde{\lambda}_{k}, k=1, \ldots, 4$.

\subsubsection{Riemann invariants}

The variables $q^{\mathrm{R}}=\left(h_{s}, \omega_{s}, h_{f}, \omega_{f}\right)^{\mathrm{T}}$ are Riemann invariants across $\lambda^{0}$,

$$
\Delta^{0} q^{\mathrm{R}}=0
$$

where $\Delta^{0}(\cdot)$ denotes increments across $\lambda^{0}$ as for the single-phase case. Then, only the momenta $m_{s}$ and $m_{f}$ can possibly have jumps across the stationary discontinuity. See the schematic representation of the Riemann solution wave structure in Fig. 1.

Across the waves propagating at speeds $\tilde{\lambda}_{k}$ we have the invariance relations:

$$
\begin{aligned}
& \tilde{\xi}_{k} h_{s}-h_{f}=\text { const., } \\
& \tilde{\lambda}_{k} h_{s}-\omega_{s}=\text { const., } \quad \tilde{\lambda}_{k} h_{f}-\omega_{f}=\text { const., } \\
& \tilde{\lambda}_{k} m_{s}-\mathcal{F}_{s}\left(\omega_{s}, h_{s}, h_{f}\right)=\text { const., } \quad \tilde{\lambda}_{k} m_{f}-\mathcal{F}_{f}\left(\omega_{f}, h_{f}\right)=\text { const. }
\end{aligned}
$$




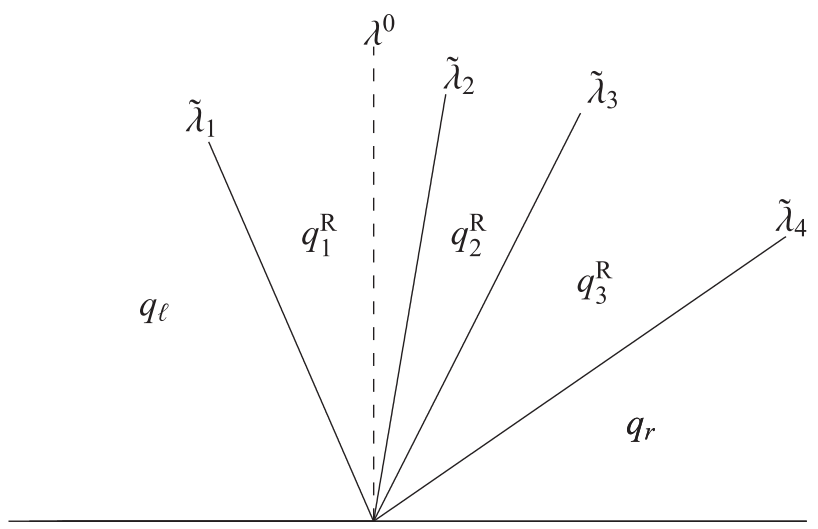

Fig. 1. Riemann solution wave structure of the two-phase relaxation model, consisting of linear waves propagating at speeds $\tilde{\lambda}_{k}, k=1, \ldots, 4$, and a stationary discontinuity $\lambda^{0}$. The variables $q^{\mathrm{R}}=\left(h_{s}, \omega_{s}, h_{f}, \omega_{f}\right)^{\mathrm{T}}$ are Riemann invariants across $\lambda^{0}$, whereas the momenta $m_{s}, m_{f}$ may have jumps across this wave.

where

$$
\mathcal{F}_{s}=\frac{\omega_{s}^{2}}{h_{s}}+\frac{1}{2} g h_{s}^{2}+g \frac{1-\gamma}{2} h_{s} h_{f}+\frac{1}{2} g \gamma \tilde{\xi}_{k} h_{s}^{2} \quad \text { and } \quad \mathcal{F}_{f}=\frac{\omega_{f}^{2}}{h_{f}}+\frac{1}{2} g h_{f}^{2}+\frac{1}{2} g \frac{h_{f}^{2}}{\tilde{\xi}_{k}} .
$$

Let us denote with $(\cdot)_{k}^{L, R}$ the states to the left and to the right of the $k$ th wave $\tilde{\lambda}_{k}, k=1, \ldots 4$, and with $\Delta_{k}(\cdot)$ the corresponding increment, $\Delta_{k}(\cdot)=(\cdot)_{k}^{R}-(\cdot)_{k}^{L}$. As before, let $\Delta(\cdot) \equiv(\cdot)_{r}-(\cdot)_{\ell}$. By using the Riemann invariants above, we can express the increments of $q$ across the considered waves by the following relations:

$$
\begin{aligned}
& \tilde{\lambda}_{k} \Delta_{k} h_{s}=\Delta_{k} \omega_{s}, \\
& \tilde{\lambda}_{k} \Delta_{k} h_{f}=\Delta_{k} \omega_{f}, \\
& \tilde{\lambda}_{k} \Delta_{k} m_{s}=\Delta_{k}\left(\frac{\omega_{s}^{2}}{h_{s}}+\frac{g}{2} h_{s}^{2}+g \frac{1-\gamma}{2} h_{s} h_{f}\right)+g \gamma \frac{h_{s, k}^{L}+h_{s, k}^{R}}{2} \Delta_{k} h_{f}, \\
& \tilde{\lambda}_{k} \Delta_{k} m_{f}=\Delta_{k}\left(\frac{\omega_{f}^{2}}{h_{f}}+\frac{g}{2} h_{f}^{2}\right)+g \frac{h_{f, k}^{L}+h_{f, k}^{R}}{2} \Delta_{k} h_{s} .
\end{aligned}
$$

Note that we can write

$$
\tilde{\lambda}_{k} \Delta_{k} q=\Delta_{k} f\left(q^{\mathrm{R}}\right)+W\left(\left(h_{f, s}\right)_{k}^{L, R}\right)
$$

where $f(q)$ is the conservative portion of the two-phase system in (10) and the term

$$
W\left(\left(h_{f, s}\right)_{k}^{L, R}\right)=\left(0, g \gamma \frac{h_{s, k}^{L}+h_{s, k}^{R}}{2} \Delta_{k} h_{f}, 0, g \frac{h_{f, k}^{L}+h_{f, k}^{R}}{2} \Delta_{k} h_{s}\right)^{\mathrm{T}}
$$

is a contribution arising from the non-conservative term $w\left(q, \partial_{x} q\right)$. The increments of the mixture momentum are

$$
\begin{aligned}
\tilde{\lambda}_{k} \Delta_{k} m_{\mathrm{m}} & =\tilde{\lambda}_{k} \Delta_{k} m_{s}+\gamma \tilde{\lambda}_{k} \Delta_{k} m_{f}=\Delta_{k}\left(\frac{\omega_{s}^{2}}{h_{s}}+\frac{g}{2} h_{s}^{2}+g \frac{1-\gamma}{2} h_{s} h_{f}\right)+\gamma \Delta_{k}\left(\frac{\omega_{f}^{2}}{h_{f}}+\frac{g}{2} h_{f}^{2}\right)+g \gamma \Delta_{k}\left(h_{s} h_{f}\right) \\
& =\Delta_{k}\left(\frac{\omega_{s}^{2}}{h_{s}}+\gamma \frac{\omega_{f}^{2}}{h_{f}}+\frac{g}{2} h_{s}^{2}+\gamma \frac{g}{2} h_{f}^{2}+g \frac{1+\gamma}{2} h_{s} h_{f}\right),
\end{aligned}
$$

and they correspond to the jump of the mixture momentum flux $f_{\mathrm{m}}(11 \mathrm{~b})$ evaluated in $q^{\mathrm{R}}$ :

$$
\tilde{\lambda}_{k} \Delta_{k} m_{\mathrm{m}}=\Delta_{k} f_{\mathrm{m}}\left(q^{\mathrm{R}}\right) .
$$

\subsection{Two-phase relaxation Riemann solver}

Let us denote with $q^{\mathrm{E}}$ the variables of the relaxation system, $q^{\mathrm{E}}=\left(h_{s}, m_{s}, h_{f}, m_{f}, \omega_{s}, \omega_{f}\right)^{\mathrm{T}}$. The exact solution of a Riemann problem for the relaxation system with left and right data $q_{\ell}^{\mathrm{E}}, q_{r}^{\mathrm{E}}$ defines an approximate Riemann solution for the original system with data $q_{\ell}, q_{r}$. The initial data of the relaxation variables correspond to the equilibrium values: 


$$
\omega_{\theta, \ell}=m_{\theta, \ell} \quad \text { and } \quad \omega_{\theta, r}=m_{\theta, r}, \quad \theta=s, f .
$$

The Riemann solution for the variables $q^{\mathrm{R}}=\left(h_{s}, \omega_{s}, h_{f}, \omega_{f}\right)^{\mathrm{T}}$ is the solution of the linear sub-system (88). The increments of $q^{\mathrm{R}}$ across the four waves $\tilde{\lambda}_{k}$ are $\Delta_{k} q^{\mathrm{R}}=\tilde{\alpha}_{k} \tilde{r}_{k}, k=1, \ldots, 4$, where $\tilde{r}_{k}$ are the four right eigenvectors of $\widetilde{A}$, and $\tilde{\alpha}_{k}$ are the coefficients of the projection $\Delta q^{\mathrm{R}}=\sum_{k=1}^{4} \tilde{\alpha}_{k} \tilde{r}_{k}$. Introducing the two-component vectors

$$
\tilde{r}_{k}^{s} \equiv\left(\begin{array}{c}
1 \\
\tilde{\lambda}_{k}
\end{array}\right) \in \mathbb{R}^{2}
$$

we can write

$$
\Delta_{k} q^{\mathrm{R}}=\left(\begin{array}{c}
\tilde{\alpha}_{k} \tilde{r}_{k}^{s} \\
\left(\tilde{\xi}_{k} \tilde{\alpha}_{k}\right) \tilde{r}_{k}^{s}
\end{array}\right) \in \mathbb{R}^{4}
$$

and, by using the left eigenvectors $\tilde{l}_{k}$ of $\widetilde{A}$ (see (29)),

$$
\begin{aligned}
& \tilde{\alpha}_{k}=\tilde{l}_{k} \Delta q^{\mathrm{R}}=\frac{1}{P^{\prime}\left(\tilde{\lambda}_{k}\right)}\left(\tilde{\vartheta}_{s, k}\left(\tilde{\lambda}_{k}-2 \tilde{u}_{s}\right), \tilde{\vartheta}_{s, k}, \tilde{\vartheta}_{f}\left(\tilde{\lambda}_{k}-2 \tilde{u}_{f}\right), \tilde{\vartheta}_{f}\right) \Delta q^{\mathrm{R}}, \\
& \left(\tilde{\xi}_{k} \tilde{\alpha}_{k}\right)=\left(\tilde{\xi}_{k} \tilde{l}_{k}\right) \Delta q^{\mathrm{R}}=\frac{1}{P^{\prime}\left(\tilde{\lambda}_{k}\right)}\left(\tilde{\zeta}_{s}\left(\tilde{\lambda}_{k}-2 \tilde{u}_{s}\right), \tilde{\zeta}_{s}, \tilde{\zeta}_{f, k}\left(\tilde{\lambda}_{k}-2 \tilde{u}_{f}\right), \tilde{\zeta}_{f, k}\right) \Delta q^{\mathrm{R}} .
\end{aligned}
$$

Here $\tilde{\vartheta}_{s, k}, \tilde{\vartheta}_{f}$ are the quantities (29b) evaluated in $\tilde{q}$, and $\tilde{\zeta}_{s}, \tilde{\zeta}_{f, k}$ correspond to

$$
\zeta_{s}=\xi_{k} \vartheta_{s, k}=g h_{f} \quad \text { and } \quad \zeta_{f, k}=\xi_{k} \vartheta_{f}=\left(\lambda_{k}-u_{s}\right)^{2}-g\left(h_{s}+\frac{1-\gamma}{2} h_{f}\right),
$$

based on (29b) and (28b). In case one phase is absent, $\tilde{h}_{s}=0$ or $\tilde{h}_{f}=0$, we simply use the single-phase solver for the only constituent of the flowing mass. This avoids the problem of the singularity of the matrix of the eigenvectors for the case of pure solid $\left(\tilde{h}_{f}=0\right)$, which gives $P^{\prime}\left(\tilde{\lambda}_{k}\right)=0$ for $\tilde{\lambda}_{2}=\tilde{\lambda}_{3}=\tilde{u}_{f}$. Nonetheless, note that the relations above allow recovering the case $\tilde{h}_{s}=0$ in which only the fluid phase is present, whereas in writing the eigenvectors in Section 3.1.2 we assumed $h_{s}>0$.

The increments for the momenta $m_{s}, m_{f}$ are given by the relations (92c) and (92d). The resulting approximate Riemann solver for the original system (10) consists of six waves $\mathcal{W}^{k}$

$$
\mathcal{W}^{k}=\Delta_{k} q, \quad k=1, \ldots, 4, \quad \mathcal{W}^{5}=\left(0, \Delta^{0} \omega_{s}, 0,0\right)^{\mathrm{T}}, \quad \mathcal{W}^{6}=\left(0,0,0, \Delta^{0} \omega_{f}\right)^{\mathrm{T}},
$$

moving at speeds $s^{k}$ given by

$$
s^{k}=\tilde{\lambda}_{k}, k=1, \ldots, 4, \quad s^{5}=s^{6}=\lambda^{0}=0 .
$$

In terms of the $f$-waves $\mathcal{Z}^{k}=s^{k} \mathcal{W}^{k}$ we have

$$
\mathcal{Z}^{k}=\tilde{\lambda}_{k} \Delta_{k} q, \quad k=1, \ldots, 4, \quad \mathcal{Z}^{5}=\mathcal{Z}^{6}=0,
$$

where $\tilde{\lambda}_{k} \Delta_{k} q$ are obtained as explained above. We summarize here the results:

$$
\begin{aligned}
& \tilde{\lambda}_{k} \Delta_{k} h_{s}=\tilde{\lambda}_{k} \tilde{\alpha}_{k}, \\
& \tilde{\lambda}_{k} \Delta_{k} m_{s}=\Delta_{k}\left(\frac{\omega_{s}^{2}}{h_{s}}+\frac{g}{2} h_{s}^{2}+g \frac{1-\gamma}{2} h_{s} h_{f}\right)+g \gamma \frac{h_{s, k}^{L}+h_{s, k}^{R}}{2} \Delta_{k} h_{f}, \\
& \tilde{\lambda}_{k} \Delta_{k} h_{f}=\tilde{\lambda}_{k}\left(\tilde{\xi}_{k} \tilde{\alpha}_{k}\right), \\
& \tilde{\lambda}_{k} \Delta_{k} m_{f}=\Delta_{k}\left(\frac{\omega_{f}^{2}}{h_{f}}+\frac{g}{2} h_{f}^{2}\right)+g \frac{h_{f, k}^{L}+h_{f, k}^{R}}{2} \Delta_{k} h_{s} .
\end{aligned}
$$

Since we only need to specify $f$-waves and speeds, and not the waves $\mathcal{W}^{k}$ themselves, we can avoid computing the jumps of the momenta across the zero eigenvalue $\lambda^{0}, \Delta^{0} \omega_{s}$ and $\Delta^{0} \omega_{f}$, which would require knowledge of the order of $\tilde{\lambda}_{k}$ with respect to $\lambda^{0}$, and therefore a distinction between possible wave configurations. Note finally that conservation of phase masses and mixture momentum is ensured, by (92a), (92b), (96) and (97).

Remark 7.1. Although this paper does not focus on the treatment of non-conservative terms, it is important to recall the associated difficulties and potential failures of numerical methods such as the one presented here or Roe's method [10]. It is well known that a first difficulty of non-conservative hyperbolic systems is the lack of a notion of weak solution in the distributional framework. The theory of Maso-LeFloch-Murat [52] has marked an important advance by giving a rigorous definition of weak solution, based on the concept of non-conservative products as a Borel measure associated to a choice of a family of paths. A numerical scheme applied to a non-conservative system in general makes a choice, either explicitly or implicitly, of the meaning of the non-conservative products. The path-conservative methods introduced by Parés [41] are consistent by construction with the definition of non-conservative products of [52], once a family of paths has been selected. 
The implicit choice of the meaning of non-conservative terms of our relaxation solver is reflected in the relations (105), which represent the generalized Rankine-Hugoniot conditions assumed by the method, and which imply that the scheme is formally consistent with the family of straight segments. Unfortunately, consistence of a numerical scheme with a family of paths does not guarantee convergence to the weak solution associated to the selected family, as shown and discussed in [53]. Here the authors also demonstrate that the error measure is usually "small".

In view of these considerations, one must be aware of the potential convergence difficulties of our relaxation scheme (and of Roe's scheme as well) when examining numerical results for the two-phase model involving shocks. See experiments in Section 8.2.1.

Let us finally mention the very recent work [54] about shortcomings of numerical methods for non-conservative systems.

\subsection{Relaxation parameters and positivity}

For the two-phase model we must guarantee non-negativity of the variables $h_{s}$ and $h_{f}$, which is equivalent to require physical admissibility of the flow depth and of the phase volume fractions. The positivity conditions are (43). Similar to the single-phase case, we wish to define the parameters $\tilde{q}=\tilde{q}\left(\tilde{h}_{s}, \tilde{h}_{f}, \tilde{u}_{s}, \tilde{u}_{f}\right)$ so as to ensure positivity of the Riemann solution of the relaxation system. However, the two-phase case is much more difficult. The relaxation Riemann solution structure is more complex, since it involves more variables and more intermediate states, and, in addition, it is not available in explicit form in terms of $\tilde{q}$. The first problem to consider is the existence of an average state $\tilde{q}$ that could ensure positivity. Indeed here the flexibility offered by the relaxation parameters $\tilde{q}$ does not seem enough to satisfy positivity conditions for all the physically non-negative variables and for all the Riemann intermediate states. Nonetheless, we can fulfill part of them, and at least satisfy positivity for the Riemann solution values of the flow height $h=h_{s}+h_{f}$. Numerical experimentation suggests that the resulting solver can robustly model a wide range of flow conditions involving vacuum states.

Motivated by our results for the single-phase case, and by our analysis of the two-phase system's eigenvalues, we propose the following strategy for defining the relaxation averages. We begin by defining these averages as the Roe averages (39):

$$
\begin{array}{ll}
\tilde{u}_{s}=\hat{u}_{s}, & \tilde{u}_{f}=\hat{u}_{f}, \\
\tilde{h}_{s}=\hat{h}_{s}, & \tilde{h}_{f}=\hat{h}_{f},
\end{array}
$$

and we keep this choice if no negative intermediate states for $h_{s}, h_{f}$ appear in the Riemann solution of the relaxation system. Far from vacuum, Roe's averages prove to be a very efficient and stable choice in the whole hyperbolic domain, which includes the two regions $|\Delta U| \leqslant 2 a \beta$ and $|\Delta U| \geqslant 2 a$. Note that it may seem appealing to set $\tilde{u}_{s}=\tilde{u}_{f}$ in the relaxation solver, since this would always give explicit expressions of the eigenvalues and it would simplify noticeably the positivity analysis of the Riemann solution. However this choice might lead to instabilities for certain regimes, namely when $|\Delta U|$ is too large.

If a negative Riemann intermediate state for $h_{s}$ and/or $h_{f}$ is detected:

(i) We keep the definition of the relaxation average velocities as the Roe velocities, relations (106a).

(ii) We fix the ratio

$$
\frac{\tilde{h}_{s}}{\tilde{h}_{s}+\tilde{h}_{f}}=\frac{\hat{h}_{s}}{\hat{h}_{s}+\hat{h}_{f}} \equiv \hat{\varphi} \text {. }
$$

(iii) Let $\hat{a}=\sqrt{g \hat{h}}, \hat{h}=\hat{h}_{s}+\hat{h}_{f}$, and $\tilde{a}=\sqrt{g \tilde{h}}, \tilde{h}=\tilde{h}_{s}+\tilde{h}_{f}$. We look for a sufficiently large value of the relaxation parameter $\tilde{a}$ with

$\tilde{a} \geqslant \hat{a}$

that allows satisfying (achievable) positivity conditions. Then we define

$$
\tilde{h}_{s}=\hat{\varphi} \tilde{h} \quad \text { and } \quad \tilde{h}_{f}=(1-\hat{\varphi}) \tilde{h}, \quad \text { where } \tilde{h}=\frac{\tilde{a}^{2}}{g} .
$$

Let us now study positivity conditions for the intermediate states of the relaxation solver. Recalling that $h_{s}, h_{f}$ are invariant across the stationary wave $\lambda^{0}=0$, we have three intermediate states $k=1,2,3$ to examine (see Fig. 1 ). The intermediate states for $h_{s}$ and $h_{f}$ can be written as

$$
\begin{aligned}
& h_{s 1,3}=\bar{h}_{s}+\frac{\tilde{\alpha}_{1}-\tilde{\alpha}_{4}}{2} \mp \frac{\tilde{\alpha}_{2}+\tilde{\alpha}_{3}}{2}, \\
& h_{s 2}=\bar{h}_{s}+\frac{\tilde{\alpha}_{1}-\tilde{\alpha}_{4}}{2}+\frac{\tilde{\alpha}_{2}-\tilde{\alpha}_{3}}{2}, \\
& h_{f 1,3}=\bar{h}_{f}+\frac{(\tilde{\xi} \tilde{\alpha})_{1}-(\tilde{\xi} \tilde{\alpha})_{4}}{2} \mp \frac{(\tilde{\xi} \tilde{\alpha})_{2}+(\tilde{\xi} \tilde{\alpha})_{3}}{2}, \\
& h_{f 2}=\bar{h}_{f}+\frac{(\tilde{\xi} \tilde{\alpha})_{1}-(\tilde{\xi} \tilde{\alpha})_{4}}{2}+\frac{(\tilde{\xi} \tilde{\alpha})_{2}-(\tilde{\xi} \tilde{\alpha})_{3}}{2},
\end{aligned}
$$


where we have used the notation $(\bar{\cdot})=\frac{\left(\cdot e_{\ell}+(\cdot)_{r}\right.}{2}$, and where, by using the velocities (106a) and the identity (47),

$$
\begin{aligned}
& \tilde{\alpha}_{k}=\frac{1}{P^{\prime}\left(\tilde{\lambda}_{k}\right)}\left(\tilde{\vartheta}_{s, k}\left(\tilde{\lambda}_{k}-\hat{u}_{s}\right) \Delta h_{s}+\tilde{\vartheta}_{f}\left(\tilde{\lambda}_{k}-\hat{u}_{f}\right) \Delta h_{f}+\tilde{\vartheta}_{s, k} \sqrt{h_{s \ell} h_{s r}} \Delta u_{s}+\tilde{\vartheta}_{f} \sqrt{h_{f \ell} h_{f r}} \Delta u_{f}\right), \\
& \left(\tilde{\xi}_{k} \tilde{\alpha}_{k}\right)=\frac{1}{P^{\prime}\left(\tilde{\lambda}_{k}\right)}\left(\tilde{\zeta}_{s}\left(\tilde{\lambda}_{k}-\hat{u}_{s}\right) \Delta h_{s}+\tilde{\zeta}_{f, k}\left(\tilde{\lambda}_{k}-\hat{u}_{f}\right) \Delta h_{f}+\tilde{\zeta}_{s} \sqrt{h_{s \ell} h_{s r}} \Delta u_{s}+\tilde{\zeta}_{f, k} \sqrt{h_{f \ell} h_{f r}} \Delta u_{f}\right) .
\end{aligned}
$$

The quantities $(\tilde{\cdot})$ above are functions of $\tilde{a}$ :

$$
\begin{aligned}
& \tilde{\vartheta}_{s, k}=\left(\tilde{\lambda}_{k}-\hat{u}_{f}\right)^{2}-(1-\hat{\varphi}) \tilde{a}^{2}, \quad \tilde{\vartheta}_{f}=\frac{1+\gamma}{2} \hat{\varphi} \tilde{a}^{2}, \\
& \tilde{\zeta}_{s}=(1-\hat{\varphi}) \tilde{a}^{2}, \quad \tilde{\zeta}_{f, k}=\left(\tilde{\lambda}_{k}-\hat{u}_{s}\right)^{2}-\left(\hat{\varphi}+\frac{1-\gamma}{2}(1-\hat{\varphi})\right) \tilde{a}^{2},
\end{aligned}
$$

and $\tilde{\lambda}_{k}=\tilde{\lambda}_{k}\left(\tilde{a} ; \hat{u}_{s}, \hat{u}_{f}, \hat{\varphi}\right)$.

Case $\Delta \widehat{\boldsymbol{U}} \equiv \hat{\boldsymbol{u}}_{\boldsymbol{s}}-\hat{\boldsymbol{u}}_{\mathrm{f}}=\mathbf{0}$.

If $\hat{u}_{s}=\hat{u}_{f} \equiv \hat{u}$, the eigenvalues $\tilde{\lambda}_{k}$ are explicitly given:

$$
\tilde{\lambda}_{1,4}=\hat{u} \mp \tilde{a} \text { and } \tilde{\lambda}_{2,3}=\hat{u} \mp \tilde{a} \hat{\beta}
$$

where

$$
\hat{\beta}=\sqrt{(1-\hat{\varphi}) \frac{1-\gamma}{2}}
$$

Therefore, we have explicit expressions for the intermediate states above, and we can easily derive optimal bounds for $\tilde{a}$. Let us introduce

$$
\begin{aligned}
& \hat{\eta}=\hat{\varphi}(1-\gamma)+1+\gamma, \\
& B=\sqrt{h_{s \ell} h_{s r}} \Delta u_{s}+\frac{1+\gamma}{2} \sqrt{h_{f \ell} h_{f r}} \Delta u_{f}, \\
& C=(1-\hat{\varphi}) \sqrt{h_{s \ell} h_{s r}} \Delta u_{s}-\hat{\varphi} \sqrt{h_{f \ell} h_{f r}} \Delta u_{f}, \\
& K_{s}=\hat{\varphi} B+\frac{1+\gamma}{2} \frac{C}{\hat{\beta}}, \quad K_{f}=(1-\hat{\varphi}) B-\frac{C}{\hat{\beta}}, \\
& K_{2}=K_{s}+K_{f}=B-\frac{1-\gamma}{2} \frac{C}{\hat{\beta}}, \\
& \Phi=(1-\hat{\varphi}) \Delta h_{s}-\hat{\varphi} \Delta h_{f}=\frac{2 h_{\ell} h_{r}}{h_{\ell}+h_{r}} \Delta \varphi .
\end{aligned}
$$

The intermediate states for $h_{s}$ and $h_{f}$ can then be written:

$$
\begin{aligned}
& h_{s 1,3}=\bar{h}_{s}-\frac{\hat{\varphi}}{\hat{\eta}} \frac{B}{\tilde{a}} \mp \frac{1+\gamma}{2} \frac{\Phi}{\hat{\eta}}, \\
& h_{f 1,3}=\bar{h}_{f}-\frac{1-\hat{\varphi}}{\hat{\eta}} \frac{B}{\tilde{a}} \pm \frac{\Phi}{\hat{\eta}}, \\
& h_{\theta 2}=\bar{h}_{\theta}-\frac{K_{\theta}}{\hat{\eta} \tilde{a}}, \quad \theta=s, f,
\end{aligned}
$$

and the intermediate states for the flow height $h$ are:

$$
\begin{aligned}
& h_{1,3}=\bar{h}-\frac{\hat{\varphi}}{\hat{\eta}} \frac{B}{\tilde{a}} \pm \frac{1-\gamma}{2} \frac{\Phi}{\hat{\eta}}, \\
& h_{2}=\bar{h}-\frac{K_{2}}{\hat{\eta} \tilde{a}} .
\end{aligned}
$$

Let us observe that the expressions for the intermediate states above have the form $h_{(\cdot)}=d_{1}+d_{2} / \tilde{a}$. It is then clear that the condition for the existence of a bounded value of $\tilde{a}$ such that $h_{(\cdot)} \geqslant 0$ for arbitrary $d_{2}$ is that $d_{1}>0$. By inspecting the relations above, we deduce that this existence condition does not hold for the lateral states $h_{s 1,3}$ and $h_{f 1,3}$. Indeed, if for instance the initial velocity jumps are zero, then $B=0$, and $h_{s 1,3}=\bar{h}_{s} \mp \frac{1+\gamma}{2} \frac{\Phi}{\eta}, h_{f 1,3}=\bar{h}_{f} \pm \frac{\Phi}{\eta}$ might be negative when $\Delta \varphi \neq 0\left(h_{s 1}\right.$ or $h_{f 3}$ for $\Delta \varphi>0, h_{f 1}$ or $h_{s 3}$ for $\Delta \varphi<0$ ). Nonetheless, there exists a finite value of $\tilde{a}$ that ensures non-negativity of the flow height $h=h_{s}+h_{f}$ in all the Riemann solution states, and non-negativity of the middle state quantities $h_{s 2}, h_{f 2}$. This means that only the phase volume fractions of the lateral states 1 or 3 could possibly be unphysical. In the particular case $\Delta \varphi=0$, which implies $h_{\theta 1}=h_{\theta 3}=h_{\theta 2}, \theta=s, f$, positivity is fully achieved. 
We obtain the following positivity conditions for $h_{1}, h_{3}$ :

$$
\tilde{a} \geqslant \frac{B_{+}}{\min \left(D_{1}, D_{3}\right)} \equiv \tilde{a}_{1,3} \quad \text { with } D_{1,3}=\hat{\eta} \bar{h} \pm \frac{1-\gamma}{2} \Phi,
$$

and the following conditions for positivity of $h_{s 2}, h_{f 2}$, which also imply positivity for all the intermediate states $h_{\theta k}, \theta=s, f, k=1,2,3$, in the particular case $\Delta \varphi=0$ :

$$
\tilde{a} \geqslant \frac{\max \left(\hat{\varphi} B_{+}, K_{s+}\right)}{\hat{\eta} \bar{h}_{s}} \equiv \tilde{a}_{s 2}, \quad \tilde{a} \geqslant \frac{\max \left((1-\hat{\varphi}) B_{+}, K_{f+}\right)}{\hat{\eta} \bar{h}_{f}} \equiv \tilde{a}_{f 2} .
$$

Note that $\hat{\eta} \bar{h}_{s}, \hat{\eta} \bar{h}_{s}>0$, and $D_{1,3}>0$ (at least one of the initial states, $\ell$ or $r$, is nonzero). Based on (117), we finally define

$$
\tilde{a}=\max \left(\sqrt{g \hat{h}}, \tilde{a}_{1,3}, \tilde{a}_{s 2}, \tilde{a}_{f 2}\right) \text {. }
$$

Note that the condition for positivity of $h_{2}$, which is weaker than (117b), is

$$
\tilde{a} \geqslant \frac{K_{2+}}{\hat{\eta} \bar{h}} \equiv \tilde{a}_{2} .
$$

Case $\Delta \widehat{\boldsymbol{U}} \neq \mathbf{0}$.

If $\hat{u}_{s} \neq \hat{u}_{f}$ then the intermediate states depend on $\tilde{a}$ through the eigenvalues $\tilde{\lambda}_{k}\left(\tilde{a} ; \hat{u}_{s}, \hat{u}_{f}, \hat{\varphi}\right)$, which are not explicitly available, and the analysis is more difficult. First, we will assume here Roe parameters satisfying the first hyperbolic sufficient condition $|\Delta \widehat{U}|<2 \hat{a} \hat{\beta}$. Under this hypothesis, if $\tilde{a}>\hat{a}$, then both the external and the internal speeds are enlarged with respect to Roe's speeds, that is $\tilde{\lambda}_{1}<\hat{\lambda}_{1}<\hat{\lambda}_{4}<\tilde{\lambda}_{4}$, and $\tilde{\lambda}_{2}<\hat{\lambda}_{2} \leqslant \hat{\lambda}_{3}<\tilde{\lambda}_{3}$, as it can be deduced from the bounding relations (17). See Fig. 2. As in the case $\Delta \widehat{U}=0$, the analysis of the behavior of (110) and (111) as $\tilde{a}$ increases shows that $h_{k}, k=1,2,3$, and $h_{s 2}, h_{f 2}$ are non-negative for $\tilde{a}$ sufficiently large. Leading order terms in $\tilde{a}$ in the formulas can be easily seen by using the expressions of the eigenvalues (18) in terms of $c_{\mathrm{e}, \mathrm{i}}, V_{\mathrm{e}, \mathrm{i}}$, and the results of Section 3.1.1. Note in particular that $c_{\mathrm{e}, \mathrm{i}}=\mathcal{O}(a)$ as $a$ increases, $V_{\mathrm{e}, \mathrm{i}}$ are bounded by quantities independent from $a$, and that the derivatives $P^{\prime}\left(\lambda_{k}\right)$ that appear in (111) can be conveniently written as

$$
\begin{aligned}
& P^{\prime}\left(\lambda_{1,4}\right)=\mp 2 c_{\mathrm{e}}\left(\left(V_{\mathrm{e}}-V_{\mathrm{i}} \mp c_{\mathrm{e}}\right)^{2}-c_{\mathrm{i}}^{2}\right) \lesseqgtr 0, \\
& P^{\prime}\left(\lambda_{2,3}\right)= \pm 2 c_{\mathrm{i}}\left(c_{\mathrm{e}}^{2}-\left(V_{\mathrm{i}}-V_{\mathrm{e}} \mp c_{\mathrm{i}}\right)^{2}\right) \gtreqless 0,
\end{aligned}
$$

from which we deduce that $\left|P^{\prime}\left(\lambda_{k}\right)\right|=\mathcal{O}\left(a^{3}\right)$ as a grows.

Although we have bounding relations for the eigenvalues $\tilde{\lambda}_{k}$, efficient analytical estimates for $\tilde{a}$ sufficiently large for positivity are difficult to derive, and here we prefer to apply a numerical iterative procedure. We use a first estimate $\tilde{a} \widehat{U}_{\Delta=0}$ computed through the formulas (117) and (118) above for the case $\Delta \widehat{U}=\hat{u}_{s}-\hat{u}_{f}=0$. Then we take iteratively

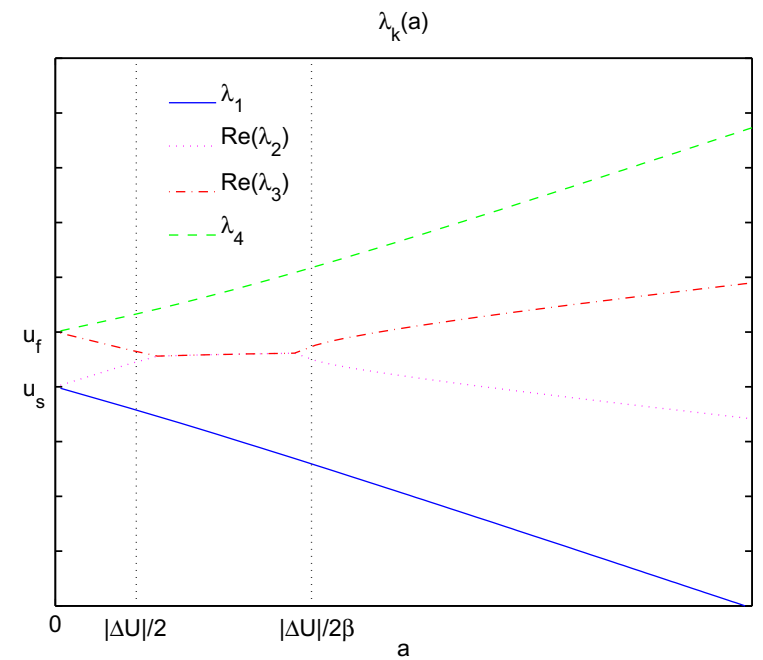

Fig. 2. Typical behaviour of the eigenvalues $\lambda_{1.4}$ and of the real part of $\lambda_{2,3}$ as a function of $a=\sqrt{g h}$ for fixed volume fraction $\varphi \neq 1$, and fixed velocities $u_{s}, u_{f}$ (assuming here $u_{f}>u_{s}$ ). In this example $\varphi=0.6, u_{s}=3, u_{f}=4, \gamma=1 / 2$ and $g=9.81$. The internal eigenvalues $\lambda_{2,3}$ become complex in a region included in the interval $\left(\frac{|\Delta U|}{2}, \frac{|\Delta U|}{2 \beta}\right),|\Delta U|=\left|u_{s}-u_{f}\right|$. 


$$
\tilde{a}^{(j)}=\tilde{a}_{\widehat{\Delta U=0}}+j b|\Delta \widehat{U}|, \quad j \in \mathbb{N}, 0 \leqslant j \leqslant n_{\max }, \quad b \in \mathbb{R}_{+},
$$

increasing the counter $j$ from 0 until positivity conditions are met, or until the eigenvalues $\tilde{\lambda}_{k}^{(j)}=\tilde{\lambda}_{k}\left(\tilde{a}^{(j)}\right)$ exceed some estimated left and right limiting speeds $c_{\ell}^{\lim }, c_{r}^{\lim }$. Practically, unphysical states appear when a rarefaction occurs at least on one side of the true Riemann solution, and on that side the external Roe speed is slower than the head of the rarefaction. Then we define $c_{\ell}^{\lim }$ and $c_{r}^{\lim }$ as the limiting speeds $\lambda_{1}\left(q_{\ell}\right)\left(h_{\ell}>0\right)$ and $\lambda_{4}\left(q_{r}\right)\left(h_{r}>0\right)$ of the rarefaction fans possibly occurring in correspondence of the characteristic fields 1 and 4 , respectively (we recall that these external fields are genuinely nonlinear [10]). In case $h_{\ell}=0$ or $h_{r}=0$, an estimate of the limiting speed of the possible rarefaction on the corresponding side is made based on the Riemann solution structure of the single-phase model with an initial dry state. In summary, we take

$$
c_{\ell}^{\lim }=\left\{\begin{array}{ll}
\lambda_{1}\left(q_{\ell}\right) & \text { if } h_{\ell}>0, \\
\min \left(u_{s r}, u_{f r}\right)-2 \sqrt{g h_{r}} & \text { if } h_{\ell}=0,
\end{array} \quad c_{r}^{\lim }= \begin{cases}\lambda_{4}\left(q_{r}\right) & \text { if } h_{r}>0, \\
\max \left(u_{s \ell}, u_{f \ell}\right)+2 \sqrt{g h_{\ell}} & \text { if } h_{r}=0,\end{cases}\right.
$$

and we stop the iteration process if

$$
\text { (a) } \tilde{\lambda}_{1}^{(j)}<c_{\ell}^{\lim }<\hat{\lambda}_{1} \text { or } \quad \text { (b) } \tilde{\lambda}_{4}^{(j)}>c_{r}^{\lim }>\hat{\lambda}_{4} \text {. }
$$

Note that if $0 \leqslant c_{\ell}^{\lim }$ for case (a), or $c_{r}^{\lim } \leqslant 0$ for case (b), then taking $\tilde{a}>\hat{a}$ has no effect on the updating formula of the solution, based on the definition of the fluctuations (34c), and we can simply keep the Roe average. This suggest that a more efficient implementation of the algorithm consists in keeping $\tilde{a}=\hat{a}$ and skipping entirely the positivity check when $0 \leqslant c_{\ell}^{\lim }<\hat{\lambda}_{1}$ or $\hat{\lambda}_{4}<c_{r}^{\lim } \leqslant 0$.

The choice of the linear growth of $\tilde{a}$ with $|\Delta \widehat{U}|$ in (121) has been suggested by the inequalities characterizing the eigenvalues (Section 3.1, rel. (24)). About the parameters, in our numerical experiments we have taken $b=1 / 8$ and $n_{\max }=12$. Typically in tests involving dry bed formation the maximum number of iterations needed is about three.

We have not developed here an algorithm specifically aimed to the case of Roe parameters in the second hyperbolic region $|\Delta \widehat{U}| \geqslant 2 \hat{a}$. Typical Riemann data do not fall in this region, nonetheless this situation may be encountered when $h \approx 0$. In this case we again increase $\tilde{a}$ with an initial estimate based on positivity conditions only on the total flow height, $\tilde{a}=\max \left(\sqrt{g \hat{h}}, \tilde{a}_{1,3}, \tilde{a}_{2}\right)$. In this second hyperbolic zone, as in the first one, the external eigenvalues are enlarged if $\tilde{a}$ grows. On the contrary, the internal eigenvalues get closer until they coincide (Fig. 2). Then we enter a complex eigenvalues region for values of $\tilde{a}$ included in the range $\left(\frac{|\Delta \widehat{U}|}{2}, \frac{|\Delta \widehat{U}|}{2 \beta}\right)$. If the estimated value of $\tilde{a}$ for positivity reaches this complex domain, we attempt to bypass it and enter the first hyperbolic region by further increasing $\tilde{a}$, subject to the stability constraints $\tilde{\lambda}_{1} \geqslant c_{\ell}^{\lim }, \tilde{\lambda}_{4} \leqslant c_{r}^{\lim }$.

In conclusion, the technique proposed here allows to guarantee non-negativity of the total flow height in the Riemann solution, but negative phase volume fractions might still appear. In such a case the computed solution will be clearly deteriorated in all the variables, but we never experienced solution blow-up. Potential difficulties related to the computation of negative volume fractions can be overcome by decreasing the Courant number. Although not rigorously positivity preserving, the relaxation method proves to model efficiently a large range of flow regimes relevant for applications involving dry bed regions. Numerical experiments will be illustrated in the next section.

\subsection{Roe-relaxation method for the two-phase model}

Following the discussion in Section 5.4 for the single-phase case, it results more efficient to apply a hybrid Roe-relaxation solver that uses the momentum waves given by the Roe solver when the relaxation parameters $\tilde{h}_{s}, \tilde{h}_{f}$ are taken as the Roe averages $\hat{h}_{s}, \hat{h}_{f}$, and the waves given by the relaxation solver otherwise. Hence, if $\tilde{a}=\hat{a}$, instead of computing the momentum increments (105b) and (105d), we use the momentum $f$-wave components of the Roe method:

$$
\begin{aligned}
& \tilde{\lambda}_{k} \Delta_{k} m_{s}=\tilde{\lambda}_{k}\left(\tilde{\lambda}_{k} \tilde{\alpha}_{k}\right), \\
& \tilde{\lambda}_{k} \Delta_{k} m_{f}=\tilde{\lambda}_{k}\left(\tilde{\lambda}_{k} \tilde{\xi}_{k} \tilde{\alpha}_{k}\right) .
\end{aligned}
$$

\subsection{The problem of complex eigenvalues}

In the previous sections we have assumed that no complex eigenvalues arise in the computation. Let us write again the sufficient condition characterizing the first hyperbolic region, which is the relevant one for the flow regimes that we model:

$$
\left|u_{s}-u_{f}\right| \leqslant 2 a \beta, \quad a=\sqrt{g h}, \quad \beta=\sqrt{(1-\varphi) \frac{1-\gamma}{2}} .
$$

Complex eigenvalues may appear when phase velocities differences are too large. In the geophysical applications of interest, we typically consider that drag forces maintain solid and fluid velocities sufficiently close, so that flow conditions are hyperbolic. However, regions of complex (internal) eigenvalues may arise even for very small $\left|u_{s}-u_{f}\right|$ when $h \rightarrow 0$ and/or $\varphi \rightarrow 1$, 
as suggested by the condition above. Then near vacuum states we face both the problem of positivity and the problem of loss of hyperbolicity.

Clearly the numerical method cannot be used if the eigenvalues of $\widetilde{A}$ are complex, and the mathematical model itself is no longer valid for flow conditions that are not hyperbolic. However, sometimes the difficulty is only numerical, and not inherent in the model system. For instance, when $h \approx 0$, occurrence of complex eigenvalues can be caused by the inaccuracy of the computed velocities, which leads to an overestimation of $\left|u_{s}-u_{f}\right|$ sufficient to enter the elliptic region. Furthermore, it may happen that initial Riemann data are hyperbolic in the whole spatial domain, but our first choice $\tilde{a}=\hat{a}$, the Roe choice of the parameters, gives complex eigenvalues for some interface Riemann problem. This indicates the tendency of the system to lose hyperbolicity, however it does not take into account drag effects, which are numerically applied in an alternate step. If drag forces are strong enough, then the true solution will remain in the hyperbolic regime everywhere as time evolves, and the numerical scheme should be able to compute it.

\subsubsection{Relaxation approach}

One natural idea to address the difficulty of complex eigenvalues is to use the flexibility of the relaxation parameters to ensure that for each local Riemann problem the matrix $\widetilde{A}$ has real eigenvalues. If we set the average phase velocities and the average volume fraction as in (106a) and (107), respectively, then we need

$$
\tilde{a} \geqslant \frac{\left|\hat{u}_{s}-\hat{u}_{f}\right|}{2 \hat{\beta}} .
$$

In practice this approach proves to be stable when initial Riemann data fall in the hyperbolic region. However, if at least one of the initial Riemann states is characterized by complex eigenvalues, then attempts to use this relaxation technique to hyperbolize interface linearized systems might lead to instabilities.

In our scheme we apply this relaxation approach (i) in the situation of hyperbolic initial Riemann states and (ii) in the general case in areas where the flow height approaches zero. When our first Roe choice $\tilde{a}=\hat{a}$ gives complex eigenvalues, the new parameter $\tilde{a}$ for hyperbolicity is defined as

$$
\tilde{a}^{\mathrm{C}}=\frac{\left|\hat{u}_{s}-\hat{u}_{f}\right|}{2 K \hat{\beta}},
$$

where $K$ is a positive parameter $<1$ ( $K=0.9$ in our tests), and the correction is made subject to the condition that resulting speeds satisfy the stability constraints $\tilde{\lambda}_{1} \geqslant c_{\ell}^{\text {lim }}$, and $\tilde{\lambda}_{4} \leqslant c_{r}^{\lim }$. Here, if $\lambda_{1}\left(q_{\ell}\right) \leqslant \hat{\lambda}_{1}$ (respectively $\left.\lambda_{4}\left(q_{r}\right) \geqslant \hat{\lambda}_{4}\right)$, which approximately indicates a rarefaction in field 1 (resp. 4), we take $c_{\ell}^{\lim }$ (resp. $c_{r}^{\lim }$ ) as the rarefaction head, as in (122). Otherwise, we allow a maximum speed enlargement $\left|\hat{\lambda}_{1}-\lambda_{1}\left(q_{\ell}\right)\right|\left(\right.$ resp. $\left.\hat{\lambda}_{4}-\lambda_{4}\left(q_{r}\right)\right)$, hence $c_{\ell}^{\lim }=2 \hat{\lambda}_{1}-\lambda_{1}\left(q_{\ell}\right)\left(\right.$ resp. $c_{r}^{\lim }=$ $2 \hat{\lambda}_{4}-\lambda_{4}\left(q_{r}\right)$ ). When we drive the solver's parameters in the first hyperbolic region, then we apply the positivity correction algorithm described previously by taking as starting estimate $\tilde{a}=\max \left(\tilde{a}^{\mathrm{C}}, \tilde{a}_{1,3}, \tilde{a}_{s 2}, \tilde{a}_{f 2}\right)$. This relaxation strategy for hyperbolicity is particularly advantageous near wet/dry fronts, where difficulties of both positivity and complex eigenvalues arise with the Roe average. In fact, increasing $\tilde{a}$ helps addressing both the issues. One disadvantage of (127) is that is looses efficiency when $\beta \rightarrow 0$ (that is $\varphi \rightarrow 1$ ). Nonetheless, this situation of appearance of complex eigenvalues with nearly pure solid does not appear to be tractable via relaxation, and alternative choices of the relaxation parameters such as the appealing $\tilde{u}_{s}=\tilde{u}_{f}$ also show to produce instabilities. Let us mention that to address this difficulty we have developed a special hyperbolizing strategy, which is not based on relaxation but uses a modified Roe-type solver that inhibits instability growth. Presentation of this approach goes beyond the purposes of the present article. In the numerical experiments illustrated in Section 8 this stabilization method has not been used.

\section{Numerical experiments}

We present in this section numerical experiments performed with the proposed relaxation solver for both the singlephase and two-phase shallow flow models. The numerical scheme has been implemented on the basis of the Fortran routines of the software CLAWPACK [55]. In all tests we set CFL $=0.9$, and we apply free flow boundary conditions. Second order corrections are applied with the Minmod limiter (cf. [13]), and with the re-limiting of the correction fluxes (34b) proposed in [44] to preserve the robustness of the first-order scheme.

Here we are interested in assessing the efficiency of the pure relaxation scheme, and we shall not present results obtained with the hybrid Roe-relaxation method. Results obtained with the two methods closely agree, but, as previously mentioned, transonic rarefactions are usually resolved more accurately by the hybrid method.

\subsection{Single-phase shallow flow model}

We begin by presenting results of two numerical experiments involving dry bed regions for the single-phase model (1). Both tests are Riemann problems, for which the initial conditions consist of two constant states separated by an interface located at $x=0$. The computational domain is $[-5,5]$, and we take 200 grid cells. The flow height $h$ is considered zero (dry bed) below the tolerance $\epsilon=10^{-6}$. 


\subsubsection{Rarefaction into vacuum}

We solve a classical test problem, considered for instance in [14,20], which contains an initial dry bed state. Left and right Riemann data are $h_{\ell}=1, h_{r}=0$ (right dry bed), $u_{\ell}=u_{r}=0$. The gravity constant is $g=1$. The exact solution of this problem consists of a single transonic rarefaction into vacuum associated with the left eigenvalue $\lambda_{1}$. Second order results for the flow height $h$ and for the momentum $m=h u$ at $t=1$ are displayed in Fig. 3. Furthermore, in Fig. 5(a) we show a zoom of the flow front zone. The computed solution is compared with the exact solution (solid line), and accurate agreement is observed. The transonic rarefaction can be resolved only if an entropy fix is applied (a small glitch in the solution is still visible at $x=0$ ).

\subsubsection{Dry bed formation}

We consider a test proposed by Toro in [14] showing the generation of a dry bed region. The initial Riemann data are $h_{\ell}=h_{r}=0.1, u_{\ell}=-3, u_{r}=3$, and we set $g=9.81$. The exact solution of this problem consists of two opposite rarefaction waves that form a dry bed area in the middle. This is a typical test for which classical Roe solver fails by computing negative values of the flow height (cf. [42,14,13]). Right at the first time step Roe solver computes a negative intermediate state $h_{\mathrm{Roe}}^{*}=-0.2029$ for the Riemann problem corresponding to the initial discontinuity at $x=0$. This does not lead immediately to unphysical cell values of $h$. Nonetheless, Roe solver crashes after few time steps by computing negative $h$ at the two cells adjacent to the initial interface, even when the computation is performed with CFL number very small $($ e.g. $C F L=0.1)$.

The relaxation solver overcomes these difficulties and is able to preserve positivity and to approximate efficiently the problem solution. First order and second order results of the relaxation scheme at $t=1$ for $h$ and $m=h u$ are shown in Fig. 4, where the exact solution is also displayed (solid line). In Fig. 5(b) we show a zoom of the dry bed region. We have tracked the positivity correction of the Roe speeds performed by the first-order relaxation algorithm for this test $(C F L=0.9)$. The correction is made only for the first 14 time steps (until time $t=0.1451$ ) and only for the Riemann problems corresponding to the initial discontinuity at $x=0$. The maximum absolute value of the new speeds $\left|\tilde{\lambda}_{1,4}\right|>\left|\hat{\lambda}_{1,4}\right|$ in this location is defined at the first time step, when we have $\tilde{\lambda}_{1,4}=\mp 3.000$ and $\hat{\lambda}_{1,4}=\mp 0.990$. Note that $\left|\tilde{\lambda}_{1,4}\right|$ is still less than the maximum absolute Roe speed value over the computational domain $\max _{i, k}\left|\hat{\lambda}_{k, i+1 / 2}\right|=3.990$, which determines in this test the time step $\Delta t$ at any time level $t^{n} \in[0,1]$, and which arises in the Riemann problems corresponding to the unperturbed flow regions on the left and on the right of the two opposite rarefactions (where no positivity correction is made and $\tilde{\lambda}_{k}=\hat{\lambda}_{k}$ ).

As an additional note on the behavior of Roe solver for this experiment, let us remark that the integration of an entropy fix technique to Roe's method may significantly alter the performance of the pure classical Roe solver described above. Among the various versions of the commonly used Harten-Hyman entropy fix [43], the formulation of LeVeque [37], also illustrated by Toro in [12], would cause difficulties at the first time step, since it uses the square root of the Roe intermediate state flow height for both the activation criterion and the Roe solution correction. However, alternative forms of the Harten-Hyman entropy fix may cure, sometimes for CFL number small enough, the positivity difficulties of Roe's method. In fact, the activation of an entropy fix with suitable parameters in regions close to vacuum may produce an enlargement of the numerical speeds that guarantees positivity preservation. Indeed, there exists a choice of the parameters that makes the HartenHyman correction equivalent to the HLL method, this ensuring positivity, see [56]. Nonetheless, relying on an entropy fix technique for positivity in this test typically gives poor accuracy in correspondence of the dry bed region $(h=0)$ of the true solution, where a spurious wet region with $h>0$ may be computed. Moreover, we stress that this strategy for positivity is not applicable to the two-phase model. Note also that we have checked that the entropy fix correction implemented in our relaxation solver does not intervene in the computation of this particular experiment, where in fact there are no problems of entropy violating solutions associated to transonic rarefactions. Let us also mention that in [25] the test problem presented here is solved by a modified Roe method [24,25], called MRoe, which is not rigorously positivity preserving. The authors need to reduce the CFL number to 0.8 in order to avoid the appearance of negative values of the water height. Yet the first order MRoe method leaves a small wet zone between the rarefaction waves [25, Fig. 7]. Since the MRoe method modifies the Roe

$\mathrm{h}$ at $\mathrm{t}=1$

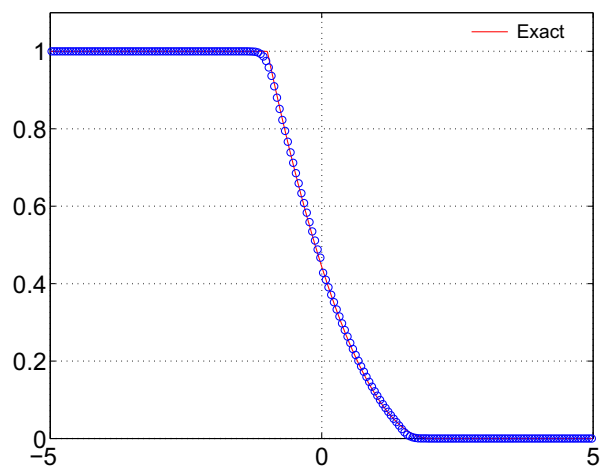

hu at $\mathrm{t}=1$

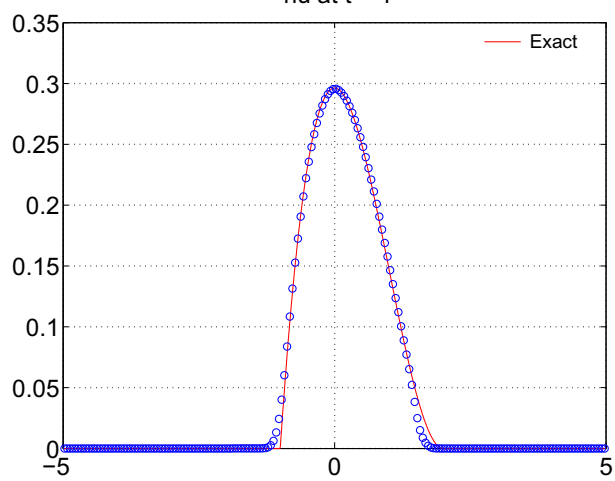

Fig. 3. Rarefaction into vacuum for the single-phase shallow flow. Flow height (left) and momentum (right) at $t=1$. 

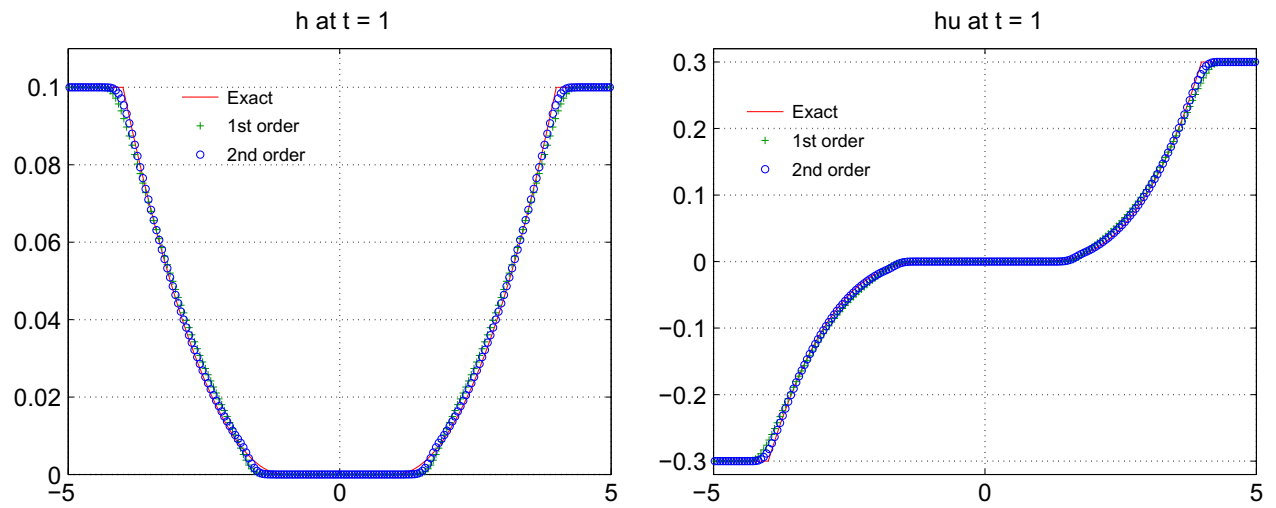

Fig. 4. Dry bed formation for the single-phase shallow flow. First-order results (pluses) and second-order results (circles) for the flow height (left) and momentum (right) at $t=1$.

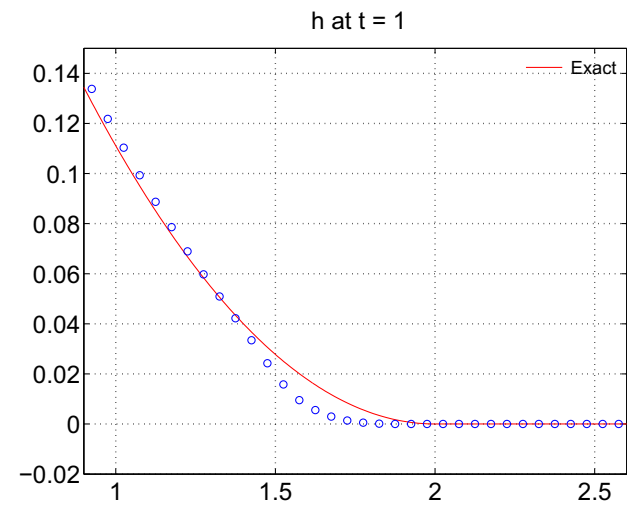

(a)

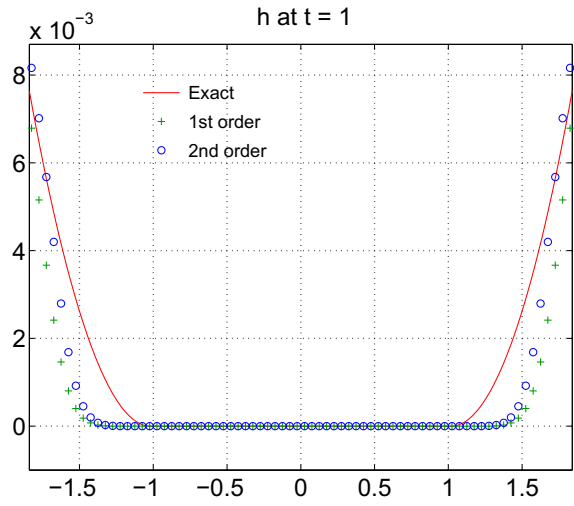

(b)

Fig. 5. Zoom of dry bed areas at $t=1$ for the two test problems for the single-phase shallow flow. (a) Flow front of the rarefaction for test in Fig. 3 ; (b) middle dry bed zone for the test in Fig. 4.

solution only when dry areas are detected, and wet/dry fronts are not produced by the MRoe method in this test, we presume that the results in [25] simply correspond to the standard Roe method with automatic (and not ad hoc) activation of the entropy fix correction, implemented by the authors in some Harten-Hyman form. This provides an example of the possible positivity preserving action of an entropy fix on Roe's computation for this experiment ( $C F L=0.8)$. Let us finally recall that in [25] the problem solution is computed accurately with no spurious wet areas by the third-order extension of the MRoe method (HMRoe).

\subsection{Two-phase shallow granular flow model}

We present now numerical results for the two-phase granular flow model (8). In all the experiments we set $\gamma=1 / 2$. As we have seen, the proposed scheme needs the eigenvalues $\tilde{\lambda}_{k}$ of the matrix $\widetilde{A}$, which are not available in explicit form. These eigenvalues are computed by Newton's iteration as explained in [10]. We will present both experiments with no drag forces and with drag forces infinitely large. Inter-phase drag terms are numerically treated by the fractional step method described in [10]. Applying infinitely large drag amounts to impose phase velocity equilibrium in the fractional step algorithm, that is each time step we reset $u_{s}=u_{f}=u_{\mathrm{eq}}$, where the equilibrium velocity is $u_{\mathrm{eq}}=\frac{h_{s} u_{s}+\gamma h_{h} u_{f}}{h_{s}+\gamma h_{f}}$ (see [10] for details). Furthermore, for tests with infinite drag the solution of the two-phase model (8) will be compared with the solution of the reduced model that can be obtained theoretically from (8) by assuming that drag forces are strong enough to drive instantaneously phase velocities to equilibrium. This model, presented in [10], consists of conservative equations for the flow height $h$, for the mass $h \rho$, and for the mixture momentum $h \rho u$, where $\rho=\varphi+\gamma(1-\varphi)$, and $u$ is the equilibrium velocity of the mixture. While for the full two-phase model exact solutions are not available (except trivial cases), this reduced model allows an easy derivation of exact Riemann solutions thanks to its simpler mathematical structure. Moreover, we have also developed a finite volume method based on a Suliciu's Riemann solver to numerically approximate the reduced model [10], so that we can solve general problems for this system. 


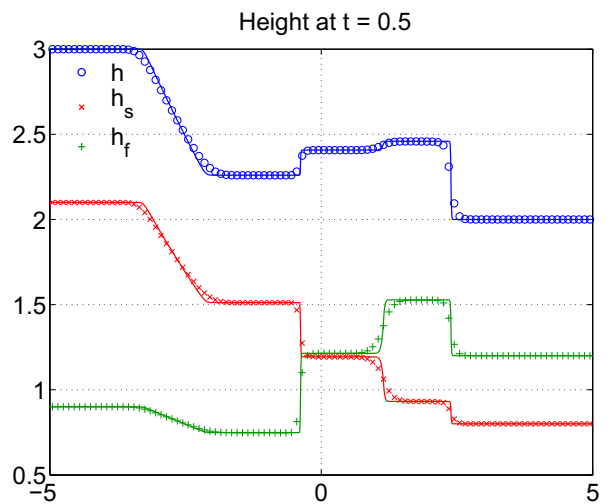

(a)

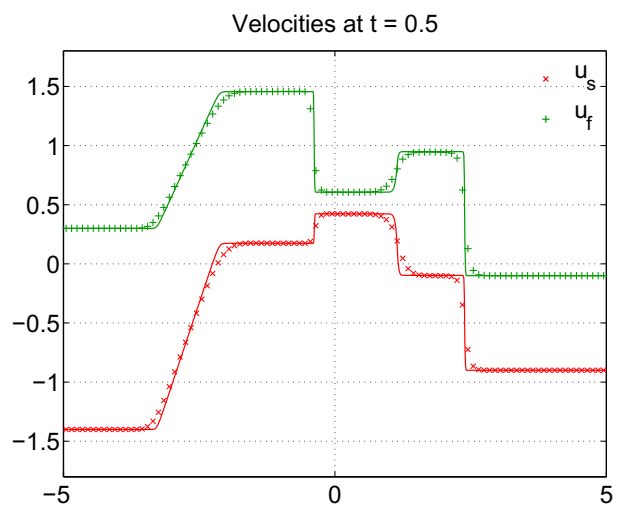

(c)

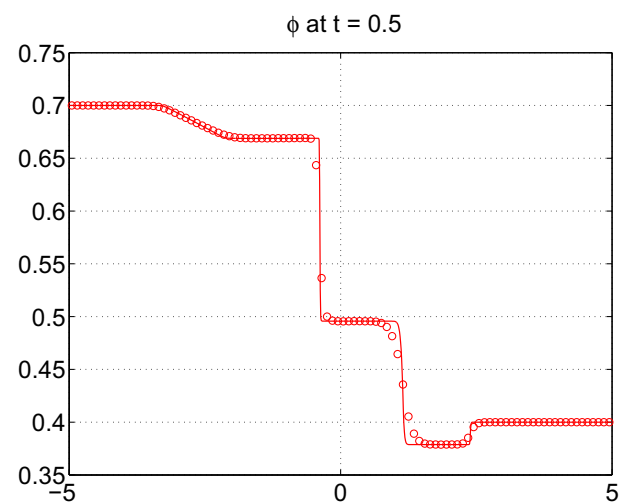

(b)

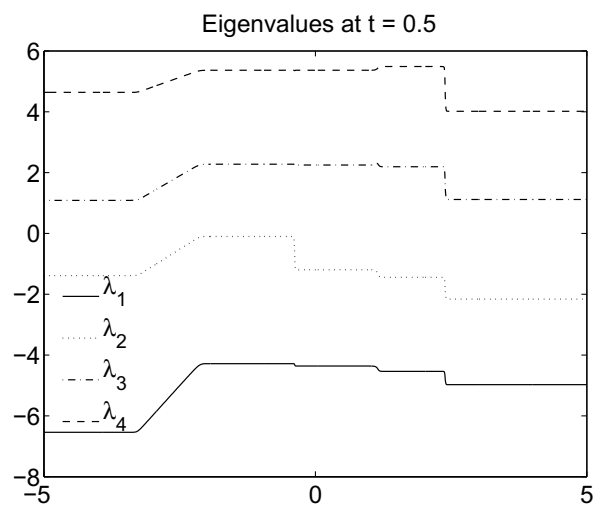

(d)

Fig. 6. Riemann problem with data (128). Sub-figures (a), (b) and (c): results at $t=0.5$ with 100 grid cells (markers) and 1000 cells (continuous line for every variable). (a) Flow height $h$ and variables $h_{s}$ and $h_{f}$; (b) solid volume fraction $\varphi$; (c) phase velocities $u_{s}$ and $u_{f}$. Sub-figure (d): Eigenvalues computed with 1000 grid cells.

Concerning the plotting style in the figures, when markers are used, we will indicate the total flow height $h$ with circles (o), the variables $h_{s}$ and $u_{s}$ for the solid phase with crosses $(\times)$, and the variables $h_{f}$ and $u_{f}$ for the fluid phase with pluses (+).

\subsubsection{Tests with no dry bed zones}

We begin by presenting results of some experiments with no dry bed areas. No drag forces are applied in these tests.

8.2.1.1. Simple Riemann problem. We consider a Riemann problem that was proposed in [10] and solved by means of the Roetype scheme. The initial discontinuity is located at $x=0$, the left and right initial data are

$$
\left(h, \varphi, u_{s}, u_{f}\right)_{\ell}=(3,0.7,-1.4,0.3) \text { and }\left(h, \varphi, u_{s}, u_{f}\right)_{r}=(2,0.4,-0.9,-0.1),
$$

and we set $g=9.81$. As in [10], we perform the computation over the interval $[-5,5]$ with 100 and 1000 grid cells. Second order results at time $t=0.5$ are shown in Fig. 6. In sub-figures (a)-(c) we display the results obtained with the two different grid resolutions for the flow height variables $h, h_{s}, h_{f}$, the solid volume fraction $\varphi$, and the phase velocities $u_{s}, u_{f}$. In subfigure (d) we plot the eigenvalues as computed with the finer grid. The Riemann solution of this problem (completely in the first hyperbolic region) consists of a 1-rarefaction, a 2-shock, a 3-rarefaction, and a 4-shock. Recalling Remark 7.1, we have to be aware of the possible convergence difficulties related to the discontinuities in the solution. Here we content ourselves by noticing agreement of our results with the results of the Roe solver in [10], and with the results computed by means of ADER schemes in [57].

8.2.1.2. Rarefaction into vacuum of the fluid constituent. We simulate a flow with $h>0$ over the whole spatial domain and over all times, but characterized by a vacuum zone for the fluid phase $\left(h_{f}=0\right)$. We consider a Riemann problem with the following data:

$$
\left(h, \varphi, u_{s}, u_{f}\right)_{\ell}=(1,0.8,0,0) \text { and }\left(h, \varphi, u_{s}, u_{f}\right)_{r}=(1,1,0,0),
$$


thus on the right there is pure solid only. The discontinuity is located a $x=0$, and $g=9.81$. As in the previous experiment, we compute the solution over [-5,5] with 100 and 1000 grid cells. Second order results are shown in Fig. 7 . The peculiarity of this problem is the occurrence of a rarefaction into vacuum for the fluid phase, which is associated to $\lambda_{2}$. This rarefaction is transonic, as we can see from the profile of the eigenvalue $\lambda_{2}$ passing through zero (Fig. 7). The Riemann solution consists of this 2-rarefaction across which $h_{f}=h(1-\varphi)$ vanishes, a 1-shock, and a 4-rarefaction occurring in pure solid material. The third wave associated to $\lambda_{3}$ is absent, similar to the problem above (Fig. 3 ) for the single-phase model, where there is not the wave corresponding to $\lambda_{2}$ in the solution pattern.

Let us finally remark that, as $\varphi$ approaches 1 , the phase velocity difference $\left|u_{s}-u_{f}\right|$ (which increases) does not satisfies the sufficient condition for hyperbolicity (125). Nonetheless, the eigenvalues of the solution states are real, and the solution evolves entirely in the first hyperbolic zone.

\subsubsection{Tests with dry bed zones}

We present now numerical results for problems that involve dry bed areas. Here the flow height $h$ is considered zero below the tolerance $\epsilon=10^{-5}$.

8.2.2.1. Spreading of a granular mass. We simulate the spreading of a granular mass on a horizontal surface. The mass is initially at rest $\left(u_{s}=u_{f}=0\right)$, and the initial profiles of the flow height and of the solid volume fraction are defined by

$$
h(x, 0)=\left\{\begin{array}{ll}
1 & \text { if } x \in[-1,1], \\
0 & \text { otherwise, }
\end{array} \text { and } \varphi(x, 0)=0.3+0.4 e^{-x^{2}} .\right.
$$

The gravity constant is $g=1$. We compute the solution for both the case of no inter-phase drag forces and the case of drag forces infinitely large, using 1000 grid cells over the domain $[-10,10]$. Second order results for the simulation without drag are displayed in Figs. 8 and 9. Fig. 8 shows the profiles of the flow height $h$ and of the solid volume fraction $\varphi$ at times $t=0,1,2,3,4$. Fig. 9 shows the eigenvalues and the phase velocity difference $\left(u_{s}-u_{f}\right)$ at time $t=4$. Note that in this problem, although there are no drag forces, the phase velocity difference approaches 0 as $h \rightarrow 0$. Hyperbolic conditions (in the first region) are then maintained as the flow height vanishes.

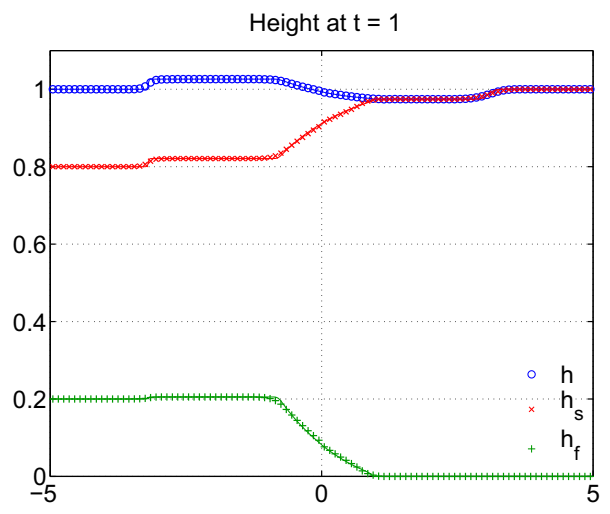

(a)

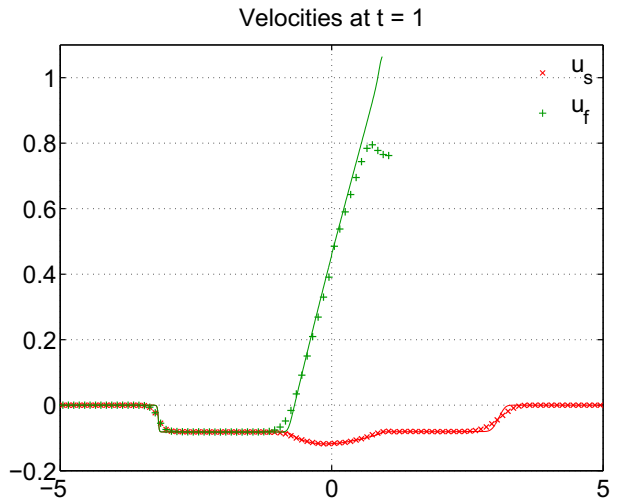

(c)

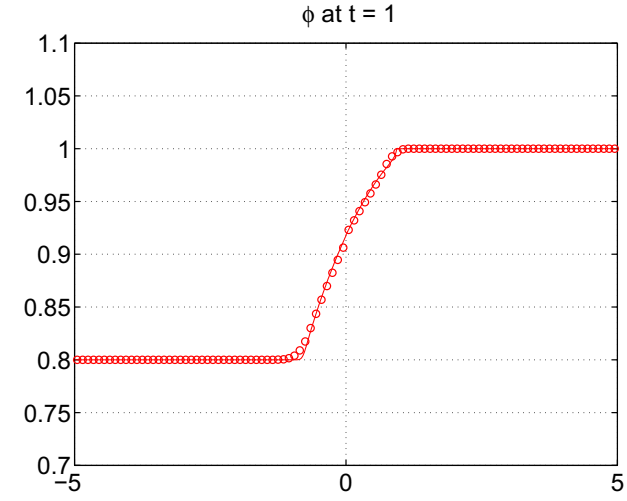

(b)

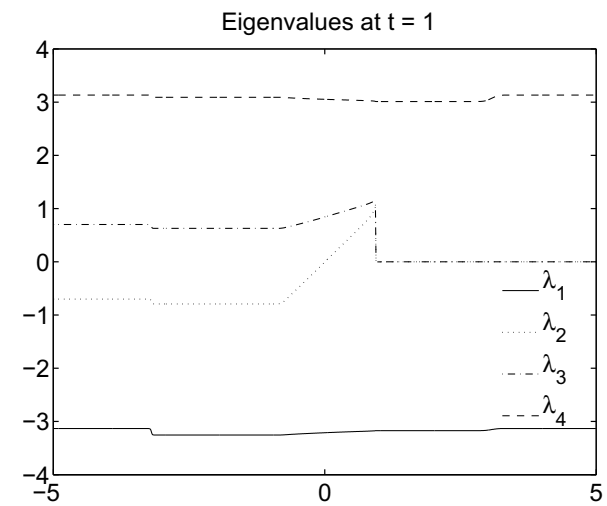

(d)

Fig. 7. Rarefaction into vacuum for the fluid constituent. Results at $t=1$ obtained with 100 and 1000 grid cells. See caption of Fig. 6 for plot description. 
$\mathrm{h}$

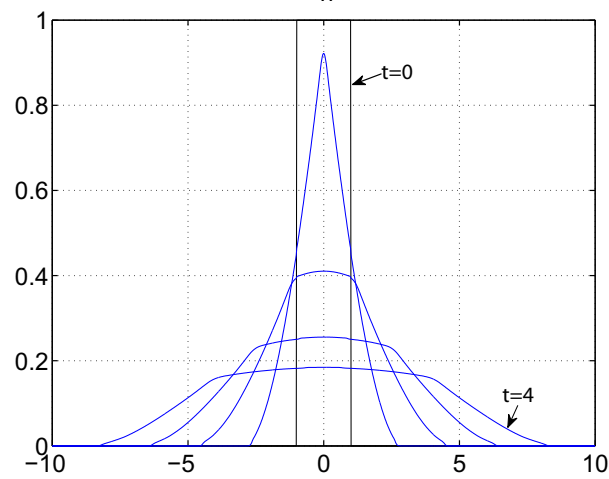

$\phi$

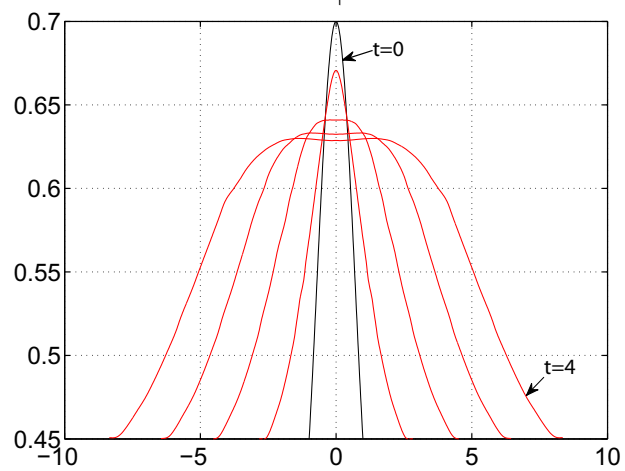

Fig. 8. Spreading of a granular mass with no drag forces. Flow height profiles (left) and solid volume fraction profiles (right) at times $t=0,1,2,3,4$.
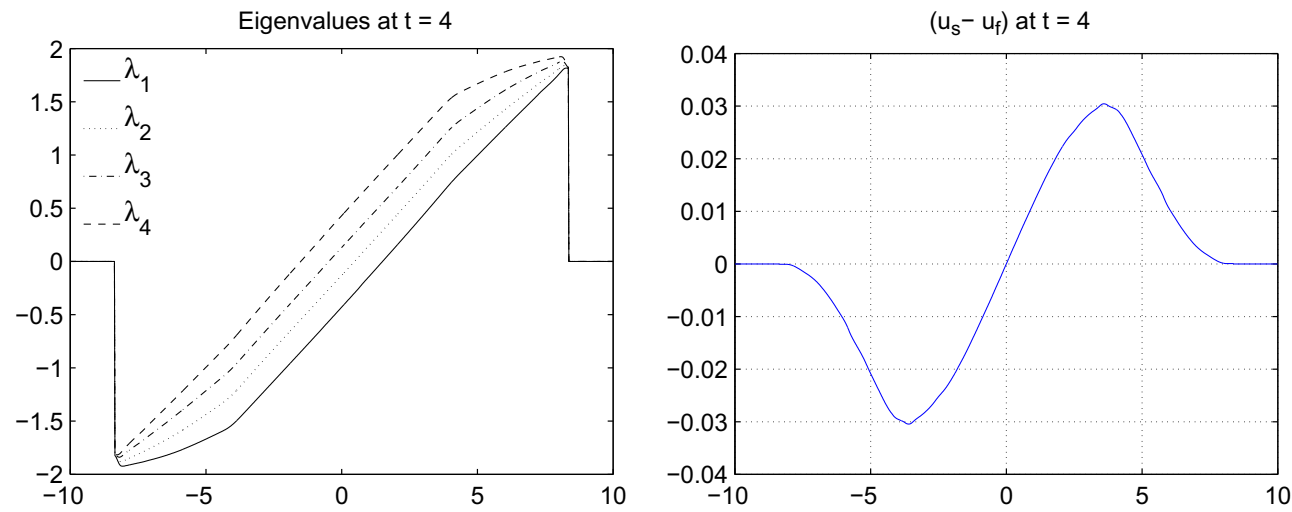

Fig. 9. Spreading of a granular mass with no drag forces. Eigenvalues (left) and phase velocity difference $\left(u_{s}-u_{f}\right)($ right $)$ at time $t=4$.

Second order results for the case of infinitely large drag are reported in Figs. 10 and 11. Fig. 10 shows profiles of $h$ and $\varphi$ at times $t=0,1,2,3,4$ for this situation, and should be compared with the case of no drag forces in Fig. 8 . We observe that the flow height dynamics is not significantly affected by the action of drag. On the other hand, the behaviour of the volume fraction varies noticeably. In Fig. 11 we plot the flow height variables, the solid volume fraction, and the phase velocities at $t=4$. Moreover, we compare these results of the two-phase model with the computed solution of the reduced model theoretically derived by assuming instantaneous kinematic equilibrium. Accurate agreement is observed between the two sets of results (lines mostly overlap). Let us finally recall that in the limit of instantaneous phase velocity equilibrium the volume fraction is simply governed by an advection equation, $\partial_{t} \varphi+u \partial_{x} \varphi=0$, where $u$ is the equilibrium flow velocity [10]. Consistently with this observation, in the case of infinite drag the profile of $\varphi$ widens laterally while preserving the initial shape as time evolves.

8.2.2.2. Dry bed generation. We solve here test problems showing the formation of a dry bed zone, which are analogous to the Toro's test presented previously for the single-phase flow. All the numerical experiments are Riemann problems whose solution consists of two opposite rarefactions that generate a dry bed region in between. We consider the following sets of Riemann data:

\begin{tabular}{lll}
\hline Test & $\left(h, \varphi, u_{s}, u_{f}\right)_{\ell}$ & $\left(h, \varphi, u_{s}, u_{f}\right)_{r}$ \\
\hline 1 & $(0.1,0.4,-3,-3)$ & $(0.1,0.7,3,3)$ \\
2 & $(0.1,0.4,0,0)$ & $(0.1,0.7,6,6)$ \\
3 & $(0.2,0.4,-3,-3)$ & $(0.1,0.8,3,3)$ \\
\hline
\end{tabular}

For all the tests the initial interface is at $x=0, g=9.81$, the computational domain is $[-5,5]$, and we use 200 grid cells. In all the simulations we apply drag forces infinitely large, so that $\left(u_{s}-u_{f}\right)$ is instantaneously driven to zero. This ensures a solution in the hyperbolic regime over the whole spatial and temporal domain. 

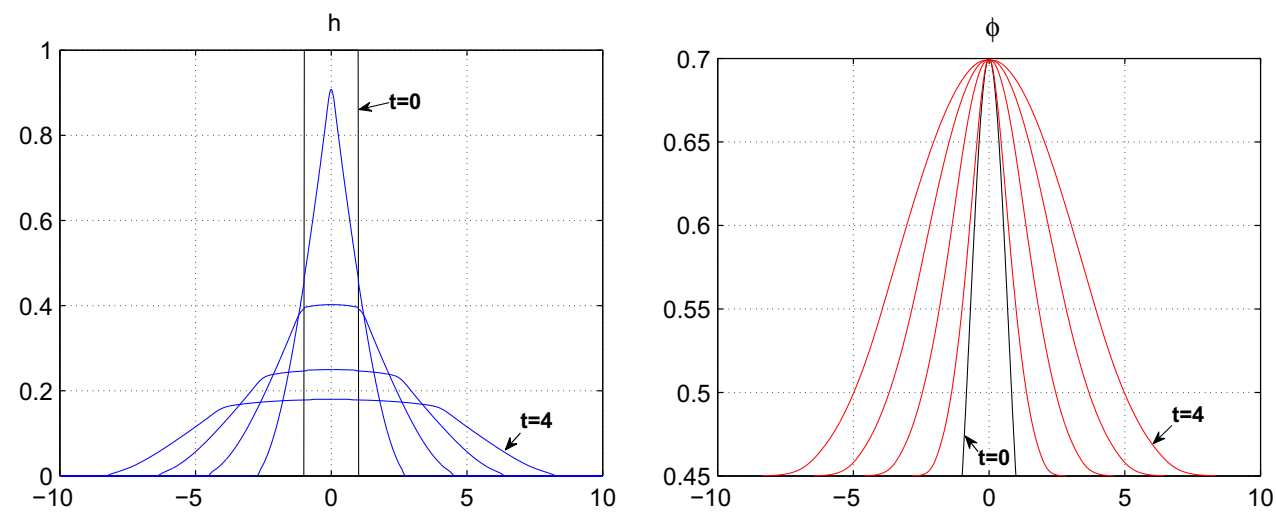

Fig. 10. Spreading of a granular mass with infinitely large drag forces. Flow height profiles (left) and solid volume fraction profiles (right) at times $t=0,1,2,3,4$.
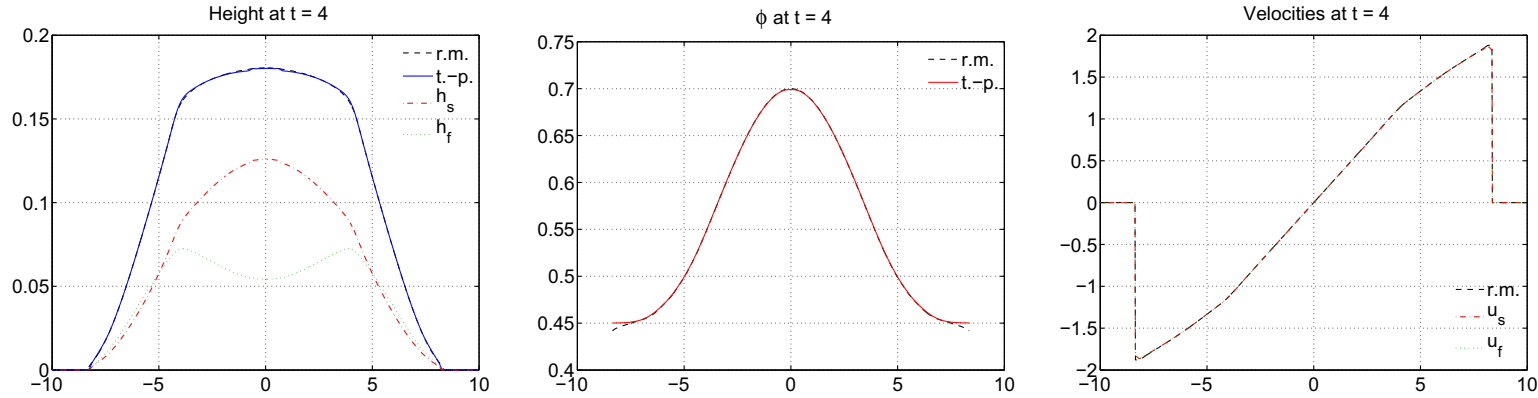

Fig. 11. Spreading of a granular mass with infinitely large drag forces. Variables $h, h_{s}, h_{f}$ (left), solid volume fraction $\varphi$ (middle) and phase velocities $u_{s}, u_{f}$ (right) at time $t=4$. Results are compared with the solution $h, \varphi, u$ (equilibrium velocity) of the reduced model (dashed line).

Test 1 is analogous to the problem in Fig. 4 for the single-phase case, except that here there is an initial discontinuity in the solid volume fraction. Second order results at $t=1$ are reported in Fig. 12. In sub-figure (a) we plot the flow height $h$ and the variables $h_{s}$ and $h_{f}$, in sub-figure (b) the phase momenta $m_{s}, m_{f}$, and the momentum of the mixture $m_{\mathrm{m}}=m_{s}+\gamma m_{f}$. A zoom of the dry bed zone from sub-figure (a) is shown in sub-figure (e). The solid volume fraction, the phase velocities and the eigenvalues are displayed in sub-figure (c), (d), and (f), respectively. The variables $h, m_{\mathrm{m}}, \varphi$ and the phase velocities are compared with the analytical Riemann solution of the instantaneous kinematic equilibrium reduced model (dashed line) for the corresponding variables. Qualitative agreement is observed.

Test 2 is similar to Test 1, except that we consider a translation of the initial velocities so that the left rarefaction is transonic. Test 3 shows a case in which there is both an initial discontinuity in the solid volume fraction and in the flow height. Second order results for these numerical experiments are plotted in Fig. 13, for Test 2, and Fig. 14, for Test 3. For each experiment we also plot the corresponding exact Riemann solution of the reduced model.

Our relaxation scheme is able to preserve non-negativity of the physically non-negative variables. Inaccuracies of phase volume fractions and phase velocities are observed near wet/dry fronts, but poor resolution of these derived variables in areas where $h \approx 0$ is a typical drawback of numerical schemes that use updating of mass (flow height) and momentum variables.

For tests simulating dry bed formation as those reported here the Roe-type method of [10] fails. The difficulties of the Roe-type solver in this case are not only related to the computation of unphysical negative states, but also to the generation of complex eigenvalues. Indeed for the three experiments presented here the Roe-type solver cannot even advance of a single time step, since the local Roe average matrix $\widehat{A}$ corresponding to the initial discontinuity at $x=0$ gives complex eigenvalues at $t=0$. In fact, although clearly all local initial Riemann states are in the hyperbolic domain since $u_{s}=u_{f}$ in each grid cell at initial time, the Roe velocities $\hat{u}_{s}, \hat{u}_{f}$ for Riemann problems at the interface $x=0$ are different, since their definition is weighted with different left and right volume fractions. The corresponding average velocity differences $|\Delta \widehat{U}|=\left|\hat{u}_{s}-\hat{u}_{f}\right|$ are sufficiently large compared to $2 \hat{a} \hat{\beta}$ to produce internal complex eigenvalues. For instance, for Test 1 , the Roe averages at $x=0$ and $t=0$ give $2 \hat{a} \hat{\beta}=0.66441,|\Delta \widehat{U}|=0.93173$, and we obtain complex eigenvalues $\hat{\lambda}_{2,3}=-0.10898 \mp 0.22026 i$. The flexibility of the relaxation solver allows throughout the computation using suitable local parameters $\tilde{a} \geqslant \hat{a}$ that ensure both real eigenvalues and positivity preservation. Here only the relaxation approach is used to overcome the problem of 


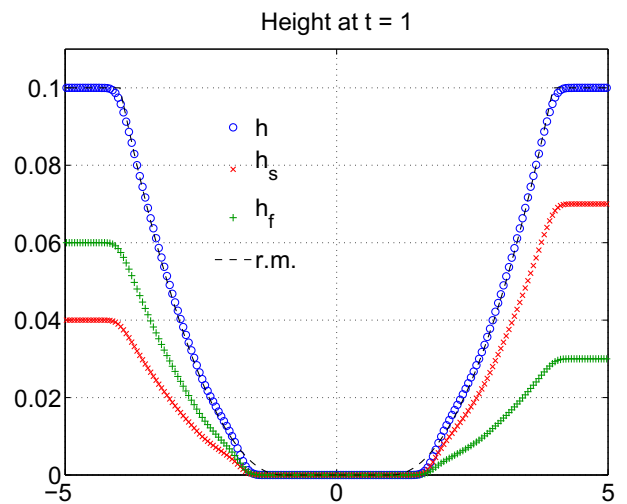

(a)

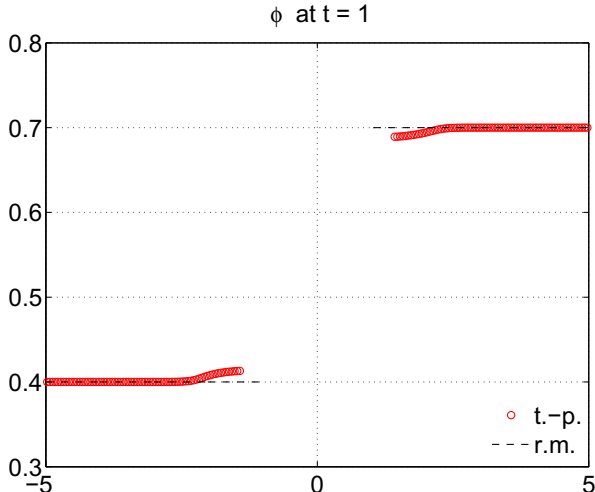

(c)

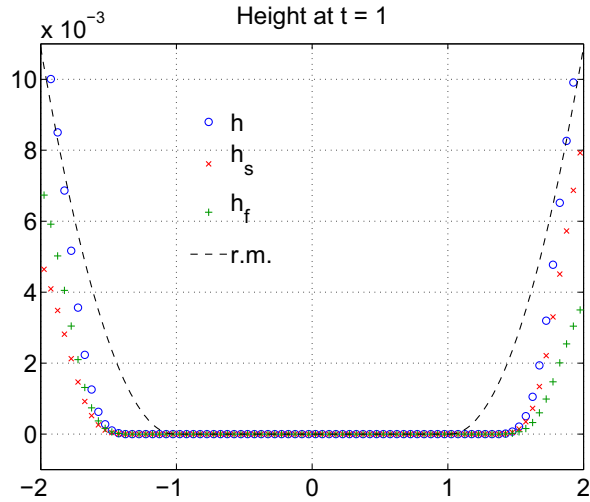

(e)

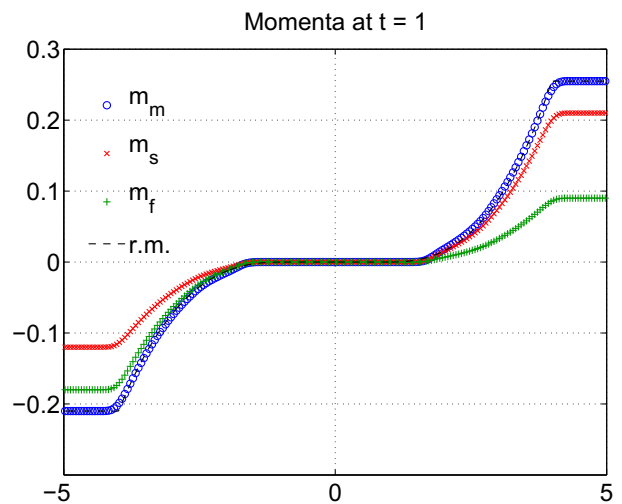

(b)

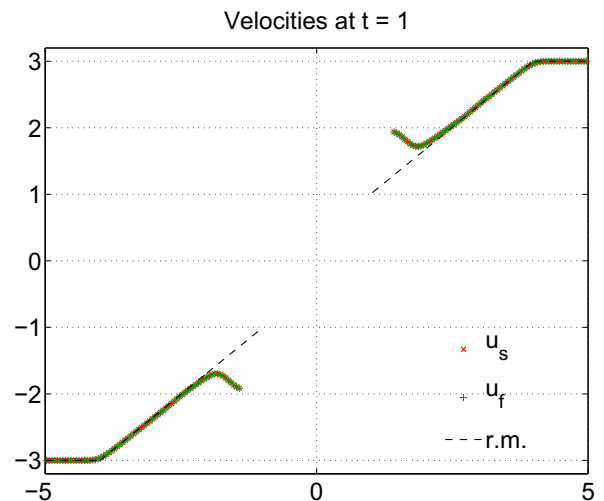

(d)

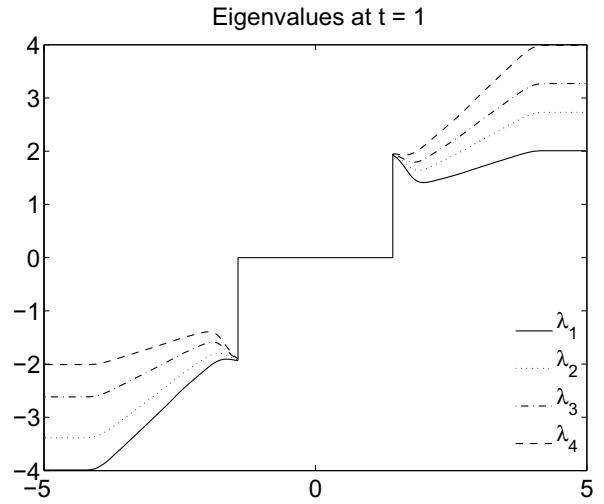

(f)

Fig. 12. Dry bed formation, Test $1, t=1$. (a): Variables $h, h_{s}, h_{f} ;$ (b) momenta $m_{s}, m_{f}, m_{\mathrm{m}} ;$ (c) solid volume fraction $\varphi$; (d) phase velocities $u_{s}, u_{f} ;(\mathrm{e})$ zoom of the dry bed region from sub-figure (a); (f) eigenvalues. The dashed line in sub-figures (a), (b), (c), (d), (e), indicates the exact solution of the reduced model for $h, h \rho u, \varphi$, and $u$, where $\rho=\varphi+\gamma(1-\varphi)$ and $u$ is the equilibrium flow velocity.

complex eigenvalues arising from Roe averages, and the hyperbolicity fix based on a modified Roe solver that we have mentioned in Section 7.5.1 does not intervene.

\section{Conclusions and extensions}

By means of a relaxation approach we have derived a new approximate Riemann solver for single-phase and two-phase shallow flow models. The resulting scheme maintains the accuracy and sharp shock resolution of Roe and Roe-type solvers, while improving significantly the robustness of Roe-type methods in handling dry bed regions. For the single-phase shallow 


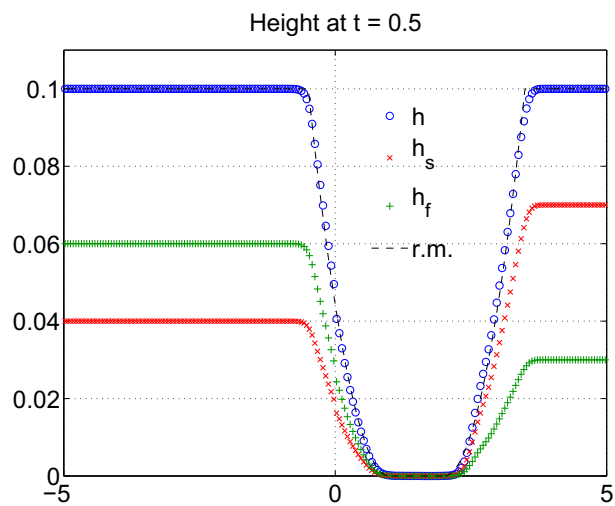

(a)

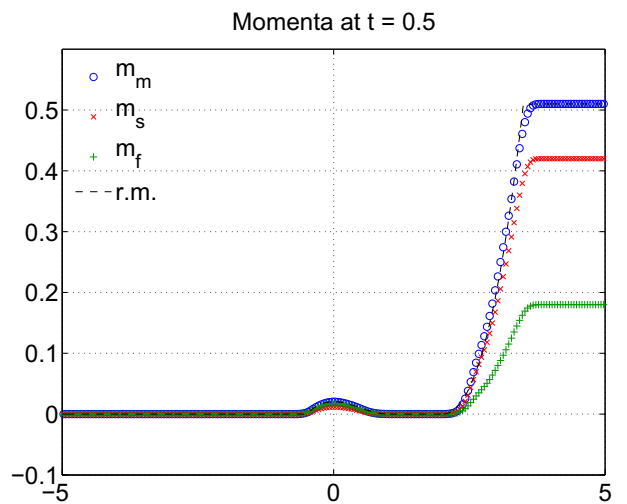

(b)

Fig. 13. Dry bed formation, Test $2, t=0.5$. (a) Variables $h, h_{s}, h_{f}$; (b) Momenta $m_{s}, m_{f}, m_{\mathrm{m}}$. Dashed line: reduced model.

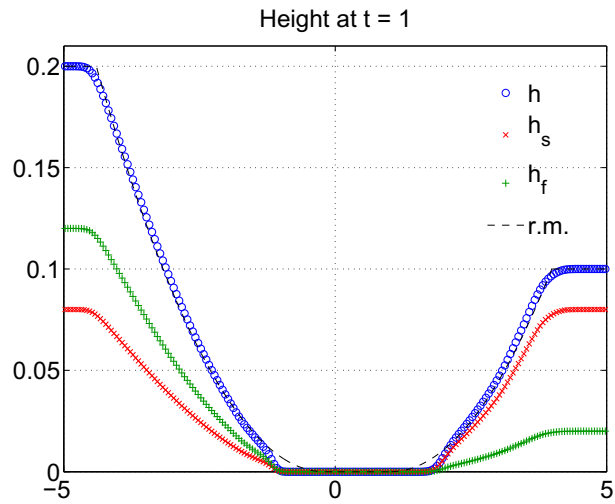

(a)

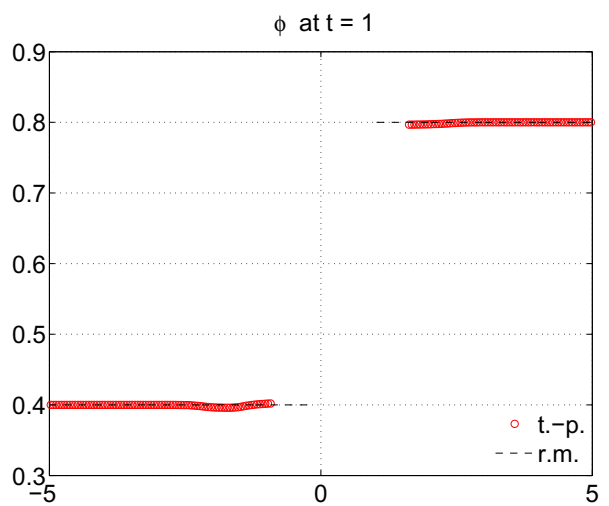

(c)

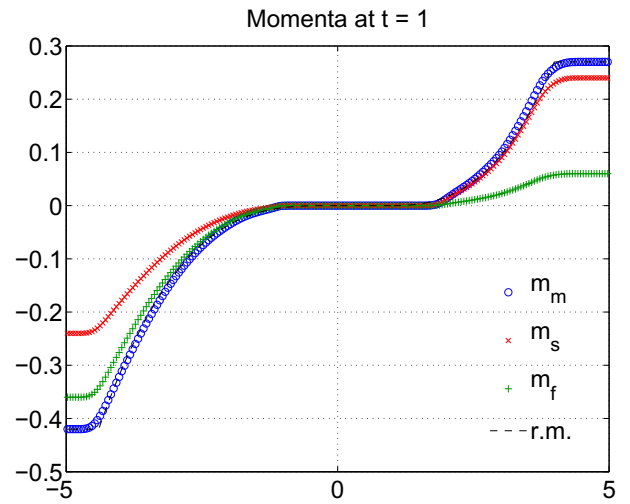

(b)

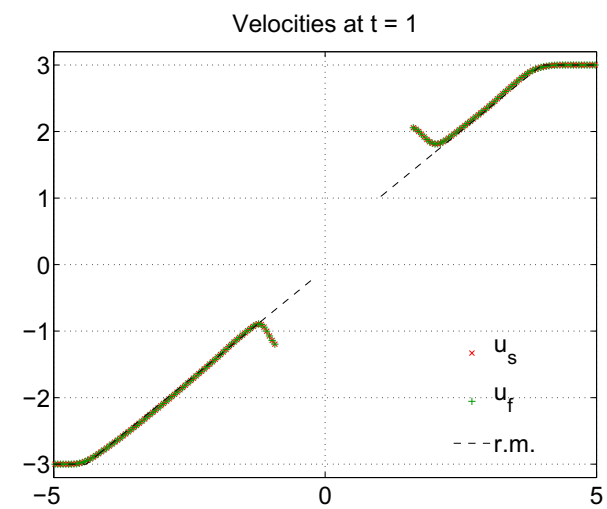

(d)

Fig. 14. Dry bed formation, Test $3, t=1$. (a) Variables $h, h_{s}, h_{f}$; (b) Momenta $m_{s}, m_{f}, m_{\mathrm{m}}$; (c) Solid volume fraction $\varphi$; (d) Phase velocities $u_{s}, u_{f}$. Dashed line: reduced model.

system, and more generally for the isentropic gas dynamics system, we obtain a scheme that rigorously preserves non-negativity of flow height (or density). The formal equivalence of the solver applied to this system with the VFRoe scheme with conservative variables of $[32,33]$ allows establishing positivity results for this VFRoe method, which originally had been considered inefficient near vacuum states. For the two-phase model positivity of phase volume fractions is not ensured, and a suitable restriction on the CFL number might be needed. Nonetheless numerical experimentation suggests that the method allows modeling effectively a wide range of flow conditions involving dry bed zones and vacuum formation. Moreover, the 
relaxation technique introduced here defines a general strategy suitable for both conservative and non-conservative systems, which could be of interest for the approximation of similar models such as the two-layer shallow flow model.

Let us mention that at the time of revising this paper we became aware of a recent work of Castro et al. [58] presenting an extension of Lax-Friedrichs scheme for general non-conservative systems that rigorously preserves positivity. We expect that our (first-order) method is less diffusive than this Lax-Friedrichs method, based on the comparative results in [58] between Lax-Friedrichs and Roe.

Our relaxation scheme for the two-phase model has been already extended to two spatial dimensions, and preliminary work has been done on adding Coulomb bottom friction forces. These results will be reported elsewhere.

Our current work focuses on the extension of the new scheme to the more general two-phase model with variable bottom topography studied in [10]. In [10] well-balanced treatment of topography source terms was performed via the $f$-wave method of $[38,23]$. This $f$-wave technique however does not seem directly applicable to the relaxation solver. Instead, the wellbalanced hydrostatic reconstruction method of Audusse et al. [59] appears a suitable option, with the advantageous property of preserving the robustness of the scheme for the homogeneous system.

\section{Acknowledgments}

The research work of the first author has been supported by a grant of the Region Île-de-France. This work was also supported by the ACI Nouvelles Interface de Mathématiques (CNRS), by the ACI Jeunes Chercheurs (CNRS), and it is part of a project funded by the French National Research Agency (ANR Blanche, BLAN-06-1_140039).

We are grateful to the anonymous reviewers for their insightful comments and valuable suggestions.

\section{Appendix A. A positivity preserving Roe matrix}

We present here a positivity preserving Roe method for single-phase shallow water equations and analogous systems of conservation laws. We consider the general form of the one-dimensional system of isentropic gas dynamics:

$$
\partial_{t} q+\partial_{x} f(q)=0
$$

where the vector $q$ and the flux function $f(q)$ are defined as

$$
q=\left(\begin{array}{c}
\varrho \\
\varrho u
\end{array}\right) \text { and } f(q)=\left(\begin{array}{c}
\varrho u \\
\varrho u^{2}+p(\varrho)
\end{array}\right) .
$$

Here $\varrho \geqslant 0$ represents a density, $u$ the velocity in the $x$ direction, and $p(\varrho)$ is a pressure function such that $p^{\prime}(\varrho)>0$. Typically, we consider a pressure law $p(\varrho)=\kappa \varrho^{\varsigma}$, with $\kappa>0$ and $\varsigma \geqslant 1$. The choice $\kappa=\frac{g}{2}, \varsigma=2$ corresponds to the shallow water system (1) (with $\varrho=h$ ). The choice $\varsigma=1$ gives the isothermal gas dynamics equations. The Jacobian matrix of the system is

$$
A(q)=f^{\prime}(q)=\left(\begin{array}{cc}
0 & 1 \\
-u^{2}+p^{\prime}(\varrho) & 2 u
\end{array}\right)
$$

The system is strictly hyperbolic under the assumption $p^{\prime}>0$, and it has eigenvalues

$$
\lambda_{1,2}=u \mp \sqrt{p^{\prime}(\varrho)} .
$$

Right and left eigenvectors are given by

$$
r_{1,2}=\left(\begin{array}{c}
1 \\
u \mp \sqrt{p^{\prime}}
\end{array}\right) \quad \text { and } \quad l_{1,2}=\frac{1}{2 \sqrt{p^{\prime}}}\left( \pm u+\sqrt{p^{\prime}}, \mp 1\right)
$$

respectively, with the normalization $l_{j} r_{k}=\delta_{j k}$.

Classically, Roe's solver defines the Roe matrix as the Jacobian matrix of the system evaluated at an average state, $\widehat{A} \equiv A(\hat{q}), \hat{q}=\hat{q}\left(\widehat{p}^{\prime}, \hat{u}\right)$. This in particular implies that the propagation speeds of the waves of the solver are the eigenvalues of the averaged Jacobian $\widehat{A}$. However, we have seen with the example of the shallow water system that this choice of $\widehat{A}$ does not let sufficient degrees of freedom for imposing both conservation and positivity. Here we propose a Roe matrix of the following form, which generalizes the classical one:

$$
\tilde{\widehat{A}} \equiv \widetilde{R}\left(\begin{array}{cc}
s^{1} & 0 \\
0 & s^{2}
\end{array}\right) \widetilde{L} .
$$

The matrices $\widetilde{R}=\left(\tilde{r}_{1}, \tilde{r}_{2}\right), \widetilde{L}=\left(\tilde{l}_{1}^{\mathrm{T}}, \tilde{l}_{2}^{\mathrm{T}}\right)^{\mathrm{T}}$ are the matrices of the right and left eigenvectors of the system evaluated in an average state $\left(\tilde{p}^{\prime}, \tilde{u}\right)$, and $s^{1}, s^{2}$ are the wave propagation speeds of this Roe solver. The matrix $\widetilde{A}$ must satisfy the conservation Roe property

$$
\tilde{\widehat{A}}\left(q_{r}-q_{\ell}\right)=f\left(q_{r}\right)-f\left(q_{\ell}\right) .
$$


In addition we require

- Non-negativity of the Riemann solution intermediate state for positivity of the scheme (Lemma 4.2):

$$
Q^{*} \geqslant 0 \text {. }
$$

- $s^{1}<s^{2}$, and $s^{1}, s^{2}$ bounded when $\varrho_{\ell} \rightarrow 0$ and $\varrho_{r}>0$, or when $\varrho_{r} \rightarrow 0$ and $\varrho_{\ell}>0$.

The idea here is to choose average values $\left(\tilde{p}^{\prime}, \tilde{u}\right)$ that guarantee positivity, and to determine the wave speeds $s^{1}, s^{2}$ by the Roe conservation condition (A.6). Note that the two waves $\mathcal{W}^{1}, \mathcal{W}^{2}$ of this new Roe solver correspond to an averaged form of the system's eigenvectors, analogously to the classical Roe solver:

$$
\mathcal{W}^{k}=\tilde{\alpha}_{k} \tilde{r}_{k}, \quad k=1,2,
$$

where the coefficients $\tilde{\alpha}_{k}$ are given by

$$
\begin{aligned}
& \tilde{\alpha}_{1}=\tilde{l}_{1} \Delta q=\frac{1}{2 \sqrt{\tilde{p}^{\prime}}}\left(\left(\tilde{u}+\sqrt{\tilde{p}^{\prime}}\right) \Delta \varrho-\Delta(\varrho u)\right), \\
& \tilde{\alpha}_{2}=\tilde{l}_{2} \Delta q=\frac{1}{2 \sqrt{\tilde{p}^{\prime}}}\left(\left(-\tilde{u}+\sqrt{\tilde{p}^{\prime}}\right) \Delta \varrho+\Delta(\varrho u)\right) .
\end{aligned}
$$

$f$-Waves for this solver are $\mathcal{Z}^{k}=s^{k} \mathcal{W}^{k}, k=1,2$. The conservation condition (A.6) can be equivalently written as

$$
\mathcal{Z}^{1}+\mathcal{Z}^{2}=s^{1} \tilde{\alpha}_{1} \tilde{r}_{1}+s^{2} \tilde{\alpha}_{2} \tilde{r}_{2}=\Delta f .
$$

For a given choice of $\left(\tilde{p}^{\prime}, \tilde{u}\right)$ these two equations form a system in the two unknowns $s^{1}, s^{2}$ :

$$
\left(\begin{array}{ll}
\tilde{\alpha}_{1} \tilde{r}_{1} & \tilde{\alpha}_{2} \tilde{r}_{2}
\end{array}\right)\left(\begin{array}{l}
s^{1} \\
s^{2}
\end{array}\right)=\Delta f .
$$

The determinant of the system's matrix is $d=2 \tilde{\alpha}_{1} \tilde{\alpha}_{2} \sqrt{\tilde{p}^{\prime}}$. If $\tilde{\alpha}_{1} \tilde{\alpha}_{2} \neq 0$, then the solution of (A.11) is uniquely determined and given by

$$
\begin{aligned}
& s^{1}=\frac{1}{\tilde{\alpha}_{1}} \tilde{l}_{1} \Delta f=\frac{1}{2 \tilde{\alpha}_{1} \sqrt{\tilde{p}^{\prime}}}\left(\left(\tilde{u}+\sqrt{\tilde{p}^{\prime}}\right) \Delta(\varrho u)-\left(\Delta\left(\varrho u^{2}\right)+\Delta p\right)\right), \\
& s^{2}=\frac{1}{\tilde{\alpha}_{2}} \tilde{l}_{2} \Delta f=\frac{1}{2 \tilde{\alpha}_{2} \sqrt{\tilde{p}^{\prime}}}\left(\left(-\tilde{u}+\sqrt{\tilde{p}^{\prime}}\right) \Delta(\varrho u)+\Delta\left(\varrho u^{2}\right)+\Delta p\right) .
\end{aligned}
$$

We now analyze the case $\tilde{\alpha}_{1} \tilde{\alpha}_{2}=0$ in which the matrix in (A.11) is singular. Such a case corresponds to the situation in which at least one wave has zero strength. First, note that $\tilde{\alpha}_{1}$ and $\tilde{\alpha}_{2}$ are both simultaneously equal to zero if and only if $\Delta q=0$. In this case waves and f-waves are simply zero vectors. Let us consider now for instance the case $\tilde{\alpha}_{1}=0$, and $\tilde{\alpha}_{2} \neq 0$. Then $\mathcal{W}^{1}=\mathcal{Z}^{1}=0$, and the Roe condition (A.10) gives

$$
\mathcal{Z}^{2}=s^{2} \mathcal{W}^{2}=s^{2} \tilde{\alpha}_{2} \tilde{r}_{2}=\Delta f \text {. }
$$

Hence the solver structure consists of a single wave $\mathcal{W}^{2}=\tilde{\alpha}_{2} \tilde{r}_{2}$ that must be collinear with $\Delta f$ through $s^{2}$. Therefore

$$
s^{2}=\frac{\Delta(\varrho u)}{\Delta \varrho}=\tilde{u}+\tilde{p}^{\prime}
$$

subject to the compatibility condition

$$
\Delta \varrho\left(\Delta\left(\varrho u^{2}\right)+\Delta p\right)=(\Delta(\varrho u))^{2} .
$$

The case $\tilde{\alpha}_{2}=0$ is analogous, and corresponds to the case of a Riemann structure made of a single wave $\mathcal{W}^{1}=\tilde{\alpha}_{1} \tilde{r}_{1}$ moving at speed

$$
s^{1}=\frac{\Delta(\varrho u)}{\Delta \varrho}=\tilde{u}-\tilde{p}^{\prime}
$$

subject again to the compatibility condition (A.15). Note that the relations above for $s^{1}, s^{2}$ are Rankine-Hugoniot jump relations.

We now look for average values $\tilde{p}^{\prime}, \tilde{u}$ that ensure the positivity condition (A.7). The strategy that we employ is analogous to the one used to define the parameters $\tilde{h}, \tilde{u}$ of our relaxation solver for single-phase shallow flow equations (Section 5.3 ) First, we take $\tilde{u}$ equal to the classical Roe-averaged velocity:

$$
\tilde{u} \equiv \hat{u}=\frac{\sqrt{\varrho_{\ell}} u_{\ell}+\sqrt{\varrho_{r}} u_{r}}{\sqrt{\varrho_{\ell}}+\sqrt{\varrho_{r}}}
$$


Then, by using the identity

$$
\Delta(\varrho u)=\hat{u} \Delta \varrho+\sqrt{\varrho_{\ell} \varrho_{r}} \Delta u,
$$

the intermediate density state has a the form

$$
\varrho^{*}=\varrho_{\ell}+\tilde{\alpha}_{1}=\varrho_{r}-\tilde{\alpha}_{2}=\frac{\varrho_{\ell}+\varrho_{r}}{2}-\frac{\sqrt{\varrho_{\ell} \varrho_{r}} \Delta u}{2 \sqrt{\tilde{p}^{\prime}}} .
$$

Therefore, we obtain the positivity condition on the parameter $\tilde{p}^{\prime}$ :

$$
\tilde{p}^{\prime} \geqslant \frac{\varrho_{\ell} \varrho_{r}(\Delta u)_{+}^{2}}{\left(\varrho_{\ell}+\varrho_{r}\right)^{2}} .
$$

With the choice $\tilde{u}=\hat{u}$ the speeds $s^{1}$ and $s^{2}$ take the form

$$
s^{1,2}=\hat{u}+\frac{\Delta p \mp \sqrt{\tilde{p}^{\prime}} \sqrt{\varrho_{\ell} \varrho_{r}} \Delta u}{\sqrt{\varrho_{\ell} \varrho_{r}} \Delta u \mp \sqrt{\tilde{p}^{\prime}} \Delta \varrho} .
$$

Furthermore, by using (A.18) and the identity

$$
\Delta\left(\varrho u^{2}\right)=\hat{u}^{2} \Delta \varrho+2 \hat{u} \sqrt{\varrho_{\ell} \varrho_{r}} \Delta u,
$$

the equality (A.15) can be rewritten as

$$
\Delta p \Delta \varrho-\varrho_{r} \varrho_{\ell}(\Delta u)^{2}=0,
$$

and the product $\tilde{\alpha}_{1} \tilde{\alpha}_{2}$ can be expressed as

$$
\tilde{\alpha}_{1} \tilde{\alpha}_{2}=-\frac{1}{4 \tilde{p}^{\prime}}\left(\varrho_{\ell} \varrho_{r}(\Delta u)^{2}-\tilde{p}^{\prime}(\Delta \varrho)^{2}\right) .
$$

Compatibility requires that whenever the above quantity $\tilde{\alpha}_{1} \tilde{\alpha}_{2}$ is zero, the condition (A.23) holds.

Let us note that the simple choice

$$
\tilde{p}^{\prime}=\widehat{p}^{\prime} \equiv \begin{cases}\Delta p / \Delta \varrho & \text { if } \Delta \varrho \neq 0, \\ p^{\prime}\left(\frac{\varrho_{\ell}+\varrho_{r}}{2}\right) & \text { if } \Delta \varrho=0\end{cases}
$$

gives speeds $s^{1}<s^{2}$ defined by

$$
s^{1,2}=\hat{u} \mp \sqrt{\widehat{p}^{\prime}} .
$$

This definition $\widehat{p}^{\prime}$ corresponds to the classical Roe average, that is it satisfies $A\left(\hat{q}\left(\widehat{p}^{\prime}, \hat{u}\right)\right) \Delta q=\Delta f$, as it can be easily verified by means of the identity $\Delta\left(\varrho u^{2}\right)=2 \hat{u} \Delta(\varrho u)-\hat{u}^{2} \Delta \varrho$. For example, for the shallow water equations $\widehat{p}^{\prime}=g \hat{\varrho}$, where $\varrho=\frac{g}{2}\left(\varrho_{r}+\varrho_{\ell}\right)$. Moreover, the choice $\widehat{p}^{\prime}$ satisfies compatibility, since

$$
\tilde{\alpha}_{1} \tilde{\alpha}_{2}= \begin{cases}\Delta \varrho\left(\Delta p \Delta \varrho-\varrho_{r} \varrho_{\ell}(\Delta u)^{2}\right) /(4 \Delta p) & \text { if } \Delta \varrho \neq 0, \\ -\frac{\varrho_{r} \varrho_{\ell}(\Delta u)^{2}}{4 p^{\prime}\left(\varrho_{\ell}+\varrho_{r} / 2\right)} & \text { if } \Delta \varrho=0,\end{cases}
$$

and clearly $\tilde{\alpha}_{1} \tilde{\alpha}_{2}=0$ implies (A.23). (84)):

In order to ensure the positivity condition (A.20), we propose the following definition for the average $\tilde{p}^{\prime}$ (anticipated in

$$
\tilde{p}^{\prime} \equiv \max \left(\hat{p}^{\prime}, \frac{\varrho_{\ell} \varrho_{r}(\Delta u)_{+}^{2}}{\left(\varrho_{\ell}+\varrho_{r}\right)^{2}}\right) .
$$

Let us now write the speeds (A.21) in terms of functions $\phi^{1,2}=\phi^{1,2}\left(\sqrt{\tilde{p}^{\prime}}\right)$ :

$$
s^{1,2}=\hat{u}+\phi^{1,2}\left(\sqrt{\tilde{p}^{\prime}}\right), \quad \phi^{1,2}\left(\sqrt{\tilde{p}^{\prime}}\right)=\frac{\Delta p \mp \sqrt{\tilde{p}^{\prime}} \sqrt{\varrho_{\ell} \varrho_{r}} \Delta u}{\sqrt{\varrho_{\ell} \varrho_{r}} \Delta u \mp \sqrt{\tilde{p}^{\prime}} \Delta \varrho} .
$$

We need to verify that for a choice $\tilde{p}^{\prime} \neq \widehat{p}^{\prime}$

$$
\partial_{\sqrt{\tilde{p}^{\prime}}} \phi^{1} \leqslant 0 \text { and } \partial_{\sqrt{\tilde{p}^{\prime}}} \phi^{2} \geqslant 0 \text {. }
$$

Straightforward computations give

$$
\partial_{\sqrt[\tilde{p}^{\prime}]{ }} \phi^{1,2}=\frac{\mp\left(\varrho_{r} \varrho_{\ell}(\Delta u)^{2}-\Delta p \Delta \varrho\right)}{\left(\sqrt{\varrho_{\ell} \varrho_{r}} \Delta u \mp \sqrt{\tilde{p}^{\prime}} \Delta \varrho\right)^{2}} .
$$



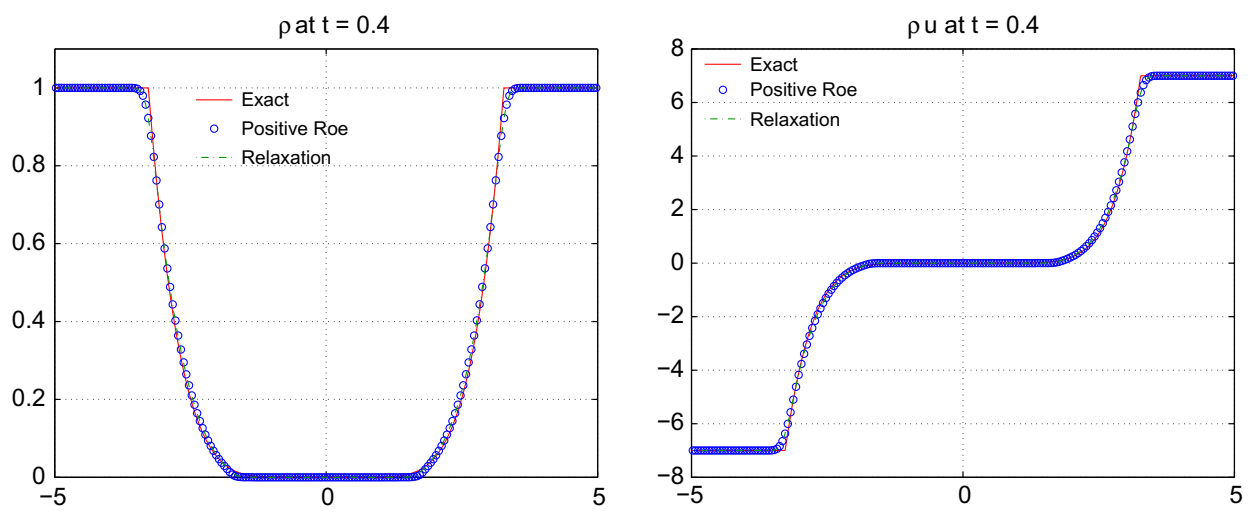

Fig. 15. Vacuum formation for the isentropic gas dynamics system with pressure function parameters $\kappa=1$ and $\varsigma=1.4$. Second order results obtained with the positive Roe method (circles) and a generalized version of the relaxation method of Section 5 (dash-dot line). Density (left) and momentum (right) at $t=0.4$.

Then the conditions (A.30) are satisfied if

$$
\Delta p \Delta \varrho-\varrho_{r} \varrho_{\ell}(\Delta u)^{2} \leqslant 0 .
$$

Note that the equality of the above quantity to zero corresponds to the compatibility condition (A.23). We easily see that, if $\Delta p \Delta \varrho-\varrho_{r} \varrho_{\ell}(\Delta u)^{2} \geqslant 0$, the definition (A.28) implies that we simply take $\tilde{p}^{\prime}=\widehat{p}^{\prime}$, observing that $(\Delta \varrho)^{2} \leqslant\left(\varrho_{r}-\varrho_{\ell}\right)^{2}$. Then the choice (A.28) allows satisfying all the required conditions.

An alternative definition for $\tilde{p}^{\prime}$ is

$$
\tilde{p}^{\prime} \equiv \widehat{p}^{\prime}+\frac{\left(\varrho_{\ell} \varrho_{r}(\Delta u)_{+}^{2}-\Delta p \Delta \varrho\right)_{+}}{\left(\varrho_{\ell}+\varrho_{r}\right)^{2}} .
$$

We leave to the reader to verify the fulfillment of positivity and compatibility conditions for this choice.

The positive Roe solver introduced here for the isentropic gas dynamics system represents a simple modification of the classical solver, which allows maintaining the good properties of the standard method (sharp resolution of shocks, low diffusivity), and in addition rigorously preserves positivity of the scheme. We have previously observed that the relaxation method introduced in Section 5 can be seen as a modified Roe method where a new definition of the momentum waves ensures conservation for any choice of the relaxation averages. For the present positive Roe solver conservation is guaranteed for any choice of the averages $\tilde{p}^{\prime}, \tilde{u}$, by means of the new definition of the propagation speeds (A.11). Let us remark that also this positive Roe method can be interpreted as a relaxation method, and the solver enters in the class of relaxation Riemann solvers introduced by LeVeque-Pelanti in [23]. The associated relaxation model is

$$
\begin{aligned}
& \partial_{t} q+\partial_{x} \Psi=0, \\
& \partial_{t} \Psi-\tilde{\widehat{A}}^{2} \partial_{x} q+2 \tilde{\widehat{A}} \partial_{x} \Psi=0,
\end{aligned}
$$

where $\Psi$ is an auxiliary vector variable approximating $f(q)$.

We have implemented for system (A.1) both the positive Roe scheme, and a generalized version of the relaxation method of Section 5 that uses relaxation parameters $\tilde{p}^{\prime}, \tilde{u}$ defined as in (A.28), (A.17) (this was mentioned in Section 6.1). We show an example in Fig. 15, where we report results for a numerical test simulating vacuum formation for a pressure function $p(\varrho)=\kappa \varrho^{\varsigma}$, with parameters $\kappa=1$ and $\varsigma=1$.4. We solve a Riemann problem with data $\varrho_{\ell}=\varrho_{r}=1, u_{\ell}=-7, u_{r}=7$ over the domain $[-5,5]$, taking 200 cells. Both schemes are efficient and results are analogous.

Although effective for system (A.1), the positive Roe method does not seem suitable for extensions to the two-phase shallow granular flow model. A first difficulty is that the two-phase system is non-conservative. While attempting to use a strategy similar to (A.10), we can write only three conservation conditions (mass of each constituent, momentum of the mixture) to determine the four wave speeds $s^{k}, k=1, \ldots, 4$. It is not clear how to devise an appropriate fourth equation to close the system for the speeds. Even so, another problem would be ensuring monotonic ordering of the speeds $s^{1}<s^{2}<s^{3}<s^{4}$. Finally, for the two-phase system it is difficult to express compatibility conditions identifying the singular case in which one or more waves have zero strength. In conclusion, the relaxation approach illustrated throughout the paper appears more advantageous for extensions to complex systems.

\section{References}

[1] E.B. Pitman, L. Le, A two-fluid model for avalanche and debris flows, Philos. Trans. Roy. Soc. A 363 (2005) 1573-1601.

[2] M. Pirulli, A. Mangeney, Result of back-analysis of the propagation of rock avalanches as a function of the assumed rheology, Rock Mech. Rock Eng. 74 (1) (2008) 59-84. 
[3] C.Y. Kuo, Y.C. Tai, F. Bouchut, A. Mangeney, M. Pelanti, R.F. Chen, K.J. Chang, Simulation of Tsaoling landslide, Taiwan, based on Saint Venant equations over general topography, Eng. Geol. 104 (2009) 181-189.

[4] P. Favreau, A. Mangeney, A. Lucas, G. Crosta, F. Bouchut, Numerical modeling of landquakes, Geophys. Res. Lett. 37 (2010) L15305, doi:10.1029/ 2010 GL043512.

[5] R.M. Iverson, The physics of debris flows, Rev. Geophys. 35 (1997) 245-296.

[6] A. Mangeney, F. Bouchut, N. Thomas, J.-P. Vilotte, M.-O. Bristeau, Numerical modeling of self-channeling granular flows and of their levee-channel deposits, J. Geophys. Res. 112, F02017, doi:10.1029/2006JF000469.

[7] N. Mangold, A. Mangeney, V. Migeon, V. Ansan, A. Lucas, D. Baratoux, F. Bouchut, Sinuous gullies on Mars: frequency, distribution, and implications for flow properties, J. Geophys. Res., in press doi:10.1029/2009JE003540.

[8] A. Lucas, A. Mangeney, Mobility and topographic effects for large Valles Marineris landslides on Mars, Geophys. Res. Lett. 34 (2007) 110201.

[9] M. Pelanti, F. Bouchut, A. Mangeney, J.-P. Vilotte, Numerical modeling of two-phase gravitational granular flows with bottom topography, in: S. Benzoni-Gavage, D. Serre (Eds.), Hyperbolic Problems: Theory, Numerics and Applications, Proceedings of the Eleventh International Conference on Hyperbolic Problems (Lyon, France, 2006), Springer, 2008, pp. 825-832.

[10] M. Pelanti, F. Bouchut, A. Mangeney, A Roe-type scheme for two-phase shallow granular flows over variable topography, ESAIM-Math. Model. Numer. 42 (2008) 851-885.

[11] E. Godlewski, P.-A. Raviart, Numerical Approximation of Hyperbolic Systems of Conservation Laws, Springer-Verlag, New York, 1996

[12] E.F. Toro, Riemann Solvers and Numerical Methods for Fluid Dynamics, Springer-Verlag, Berlin, Heidelberg, 1997.

[13] R.J. LeVeque, Finite Volume Methods for Hyperbolic Problems, Cambridge University Press, 2002.

[14] E.F. Toro, Shock-Capturing Methods for Free-Surface Shallow Flows, Wiley, 2001.

[15] J.-M. Hervouet, Hydrodynamics of Free Surface Flows: Modelling with the Finite Element Method, Wiley, 2007.

[16] S.K. Godunov, A difference method for numerical calculation of discontinuous solutions of the equations of hydrodynamics, Mat. Sb. 47 (1959) 271306.

[17] A. Harten, P.D. Lax, B. van Leer, On upstream differencing and Godunov-type schemes for hyperbolic conservation laws, SIAM Rev. 25 (1983) $35-61$.

[18] I. Suliciu, On modelling phase transitions by means of rate-type constitutive equations, shock wave structure, Int. J. Eng. Sci. 28 (1990) 829-841.

[19] I. Suliciu, Some stability-instability problems in phase transitions modelled by piecewise linear elastic or viscoelastic constitutive equations, Int. J. Eng. Sci. 30 (1992) 483-494.

[20] F. Bouchut, Nonlinear Stability of Finite Volume Methods for Hyperbolic Conservation Laws, and Well-Balanced Schemes for Sources, BirkhäuserVerlag, 2004.

[21] C. Berthon, F. Marche, A positive preserving high order VFRoe scheme for shallow water equations: a class of relaxation schemes, SIAM J. Sci. Comput. 30 (2008) 2587-2612.

[22] D.L. George, Augmented Riemann solvers for the shallow water equations with steady states and inundation, J. Comput. Phys. 227 (6) (2008) 30893113.

[23] R.J. LeVeque, M. Pelanti, A class of approximate Riemann solvers and their relation to relaxation schemes, J. Comput. Phys. 172 (2001) $572-591$.

[24] M.J. Castro, J.M. González-Vida, C. Parés, Numerical treatment of wet/dry fronts in shallow flows with a modified Roe scheme, Math. Model. Methods Appl. Sci. 16 (6) (2006).

[25] J.M. Gallardo, C. Parés, M. Castro, On a well-balanced high-order finite volume scheme for shallow water equations with topography and dry areas, J. Comput. Phys. 227 (2007) 574-601.

[26] T. Buffard, T. Gallouët, J.-M. Hérard, Un schéma simple pour les équations de Saint-Venant, C.R. Acad. Sci. Paris, Série I 323 (1998) $386-390$.

[27] T. Gallouët, J.-M. Hérard, N. Seguin, On the use of symmetrizing variables for vacuums, Calcolo 40 (2003) 163-194.

[28] T. Gallouët, J.-M. Hérard, N. Seguin, Some approximate Godunov schemes to compute shallow-water equations with topography, Comput. Fluids 32 (2003) 479-513.

[29] S. Jin, Z.P. Xin, The relaxation schemes for systems of conservation laws in arbitrary space dimensions, Commun. Pure Appl. Math. 48 (1995) $235-276$.

[30] T.P. Liu, Hyperbolic conservation laws with relaxation, Commun. Math. Phys. 108 (1987) 153-175.

[31] F. Coquel, B. Perthame, Relaxation of energy and approximate Riemann solvers for general pressure laws in fluid dynamics, SIAM J. Numer. Anal. 35 (1998) 2223-2249.

[32] T. Gallouët, J. Masella, Un schéma de Godunov approché, C.R. Acad. Sci. Paris, Série I 323 (1996) 77-84.

[33] J.M. Masella, I. Faille, T. Gallouët, On an approximate Godunov scheme, Int. J. Comput. Fluid Dyn. 12 (1999) 133-149.

[34] P.L. Roe, Approximate Riemann solvers, parameter vectors, and difference schemes, J. Comput. Phys. 43 (1981) 357-372.

[35] L.H. Le, New models for geophysical mass flows, Ph.D. Thesis, State University of New York at Buffalo, 2006.

[36] R.J. LeVeque, Wave propagation algorithms for multi-dimensional hyperbolic systems, J. Comput. Phys. 131 (1997) 327-353.

[37] R.J. LeVeque, Numerical Methods for Conservation Laws, Birkhäuser-Verlag, 1990.

[38] D. Bale, R.J. LeVeque, S. Mitran, J.A. Rossmanith, A wave-propagation method for conservation laws and balance laws with spatially varying flux functions, SIAM J. Sci. Comput. 24 (2002) 955-978.

[39] I. Toumi, A weak formulation of Roe's approximate Riemann solver, J. Comput. Phys. 102 (1992) 360-373.

[40] C. Parés, M.J. Castro, On the well-balance property of Roe's method for nonconservative hyperbolic systems. Applications to shallow-water systems, ESAIM-Math. Model. Numer. 38 (2004) 821-852.

[41] C. Parés, Numerical methods for nonconservative hyperbolic systems: a theoretical framework, SIAM J. Numer. Anal. 44 (2006) 300-321.

[42] B. Einfeldt, C.D. Munz, P.L. Roe, B. Sjögreen, On Godunov-type methods near low densities, J. Comput. Phys. 92 (1991) $273-295$.

[43] A. Harten, J.M. Hyman, Self-adjusting grid methods for one-dimensional hyperbolic conservation laws, J. Comput. Phys. 50 (1983) $235-269$.

[44] R.J. LeVeque, D.L. George, High-resolution finite volume methods for the shallow water equations with bathymetry and dry states, in: P.L.-F. Liu, H. Yeh, C. Synolakis (Eds.), Advanced Numerical Models for Simulating Tsunami Waves and Runup, Advances in Coastal and Ocean Engineering, vol. 10, World Scientific, 2008, pp. 43-73.

[45] T. Buffard, T. Gallouët, J.-M. Hérard, A naïve Riemann solver to compute a non-conservative hyperbolic system, International Series of Numerical Mathematics, vol. 129, Birkhäuser-Verlag, 1999, pp. 129-138.

[46] E. Declercq, A. Forestier, J.-M. Hérard, Comparaison de solveurs numériques pour simuler la turbulence compressible, C.R. Acad. Sci. Paris, Série I 331 (2000) 1011-1016.

[47] L.V. Ovsyannikov, Two-layer shallow water models, Prikl. Mekh. Tekh. Fiz. 2 (1979) 3-14.

[48] C.B. Vreugdenhil, Two-layer shallow-water flow in two dimensions, a numerical study, J. Comput. Phys. 33 (1979) 169-184.

[49] M.J. Castro, J. Macías, C. Parés, A Q-scheme for a class of systems of coupled conservation laws with source term. Application to a two-layer 1-D shallow water system, ESAIM-Math. Model. Numer. 35 (2001) 107-127.

[50] R. Abgrall, S. Karni, Two-layer shallow water systems: a relaxation approach, SIAM J. Sci. Comput. 31 (2009) 1603-1627.

[51] T. Buffard, T. Gallouët, J.-M. Hérard, A sequel to a rough Godunov scheme: application to real gases, Comput. Fluids 29 (2000) 813-847.

[52] G.D. Maso, P.G. LeFloch, F. Murat, Definition and weak stability of nonconservative products, J. Math. Pure Appl. 74 (1995) $483-548$.

[53] M.J. Castro, P. LeFloch, M.L. Muñoz-Ruiz, C. Parés, Why many theories of shock waves are necessary: convergence error in formally path-consistent schemes, J. Comput. Phys. 227 (2008) 8107-8129.

[54] R. Abgrall, S. Karni, A comment on the computation of non-conservative products, J. Comput. Phys. 229 (8) (2010) 2759-2763.

[55] R.J. LeVeque, CLAWPACK, <http://www.amath.washington.edu/claw>.

[56] M. Pelanti, L. Quartapelle, L. Vigevano, Low dissipation entropy fix for positivity preserving Roe's scheme, in: E.F. Toro (Ed.), Godunov Methods: Theory and Applications, Kluwer/Plenum Academic Press, 2001, pp. 685-690. 
[57] M. Dumbser, M. Castro, C. Parés, E.F. Toro, ADER schemes on unstructured meshes for nonconservative hyperbolic systems: applications to geophysical flows, Comput. Fluids 38 (2009) 1731-1748.

[58] M.J. Castro, A. Pardo, C. Parés, E.F. Toro, On some fast well-balanced first order solvers for nonconservative systems, Math. Comput. 79 (2010) $1427-$ 1472.

[59] E. Audusse, F. Bouchut, M.-O. Bristeau, R. Klein, B. Perthame, A fast and stable well-balanced scheme with hydrostatic reconstruction for shallow water flows, SIAM J. Sci. Comput. 25 (2004) 2050-2065. 